\title{
THE SPITZER SURVEY OF INTERSTELLAR CLOUDS IN THE GOULD BELT. II. THE CEPHEUS FLARE OBSERVED WITH IRAC AND MIPS
}

\author{
Jason M. Kirk ${ }^{1}$, Derek Ward-Thompson ${ }^{1}$, James Di Francesco ${ }^{2}$, Tyler L. Bourke ${ }^{3}$, Neal J. Evans, II ${ }^{4}$, Bruno Merín ${ }^{5}$, \\ Lori E. Allen ${ }^{3}$, Lucas A. Cieza ${ }^{6}$, Michael M. Dunham ${ }^{4}$, Paul Harvey ${ }^{4}$, Tracy Huard ${ }^{7}$, Jes K. Jørgensen ${ }^{8}$, \\ Jennifer F. Miller ${ }^{7}$, Alberto Noriega-Crespo ${ }^{9}$, Dawn Peterson ${ }^{3}$, Tom P. Ray ${ }^{10}$, and Luisa M. Rebull ${ }^{9}$ \\ ${ }^{1}$ School of Physics and Astronomy, Cardiff University, Queens Buildings, The Parade, Cardiff, CF24 3AA, UK; \\ jason.kirk@astro.cf.ac.uk, derek.ward-thompson@astro.cf.ac.uk \\ 2 National Research Council of Canada, Herzberg Institute of Astrophysics, 5071 West Saanich Road, Victoria, BC V9E 2E7, Canada; \\ james.difrancesco@nrc-cnrc.gc.ca \\ ${ }^{3}$ Smithsonian Astrophysical Observatory, 60 Garden Street, MS42, Cambridge, MA 02138, USA; \\ bourke@cfa.harvard.edu, leallen@cfa.harvard.edu,dpeterson@cfa.harvard.edu \\ ${ }^{4}$ Department of Astronomy, University of Texas at Austin, 1 University Station, C1400, Austin, TX 78712-0259, USA; \\ nje@bubba.as.utexas.edu,mdunham@astro.as.utexas.edu,pmh@astro.as.utexas.edu \\ ${ }^{5}$ Herschel Science Centre, European Space Astronomy Center (ESA), P.O. Box 78, E-28691 Villanueva de la Cañada, Madrid, Spain; Bruno.Merin@ sciops.esa.int \\ ${ }^{6}$ Institute for Astronomy, University of Hawaii, Manoa, HI 96822, USA; 1cieza@ifa.hawaii.edu \\ ${ }^{7}$ Astronomy Department, University of Maryland, College Park, MD 20742, USA; thuard@astro.umd.edu, jfm@astro.umd.edu \\ ${ }^{8}$ Argelander-Institut für Astronomie, University of Bonn, Auf dem Hügel 71, 53121 Bonn, Germany; jes@ astro.uni-bonn.de \\ ${ }^{9}$ Spitzer Science Center, California Institute of Technology, Pasadena, CA 91125, USA; alberto@ipac.caltech.edu, rebull@ipac.caltech.edu \\ ${ }^{10}$ School of Cosmic Physics, Dublin Institute for Advanced Studies, 5 Merrion Square, Dublin 2, Ireland; tr@cp.dias.ie \\ Received 2009 February 13; accepted 2009 August 18; published 2009 November 3
}

\begin{abstract}
We present Spitzer Infrared Array Camera (IRAC; $22 \mathrm{deg}^{2}$ ) and Multiband Imaging Photometer for Spitzer (MIPS; $\sim 8 \mathrm{deg}^{2}$ ) observations of the Cepheus Flare, which is associated with the Gould Belt, at an approximate distance of $\sim 300$ pc. Around 6500 sources are detected in all four IRAC bands, of which $\sim 900$ have MIPS $24 \mu \mathrm{m}$ detections. We identify 133 young stellar object (YSO) candidates using color-magnitude diagram techniques, and a large number of the YSO candidates are associated with the NGC 7023 reflection nebula. Cross-identifications were made with the Guide Star Catalog II and the IRAS Faint Source Catalog, and spectral energy distributions (SEDs) were constructed. SED modeling was conducted to estimate the degree of infrared excess. It was found that a large majority of disks were optically thick accreting disks, suggesting that there has been little disk evolution in these sources. Nearestneighbor clustering analysis identified four small protostellar groups (L1228, L1228N, L1251A, and L1251B) with 5-15 members each and the larger NGC 7023 association with 32 YSO members. The star-formation efficiency for cores with clusters of protostars and for those without clusters was found to be $\sim 8 \%$ and $\sim 1 \%$, respectively. The cores L1155, L1241, and L1247 are confirmed to be starless down to our luminosity limit of $L_{\text {bol }}=0.06 L_{\odot}$.
\end{abstract}

Key words: infrared: general - ISM: individual (Cepheus Flare) - stars: formation

Online-only material: color figures, machine-readable tables

\section{INTRODUCTION}

The Gould Belt is a band of stars and molecular clouds that encircle the sky at an inclination of $\sim 20^{\circ}$ to the Galactic plane (Herschel 1847; Gould 1879). It is the locus of star formation within $140-500 \mathrm{pc}$ of the Sun and includes many well-known star-formation regions (Serpens, Ophiuchus, Orion, etc.). The Spitzer Gould Belt Survey (SGBS; L. Allen et al. 2009 , in preparation) is a Spitzer GO-4 legacy project designed to extend the earlier successful Spitzer Cores to Disks (c2d; Evans et al. 2003, 2009) legacy project and complete a census of star formation within $500 \mathrm{pc}$. In addition, SGBS and c2d data complement data from the upcoming James Clerk Maxwell Telescope (JCMT; Ward-Thompson et al. 2007) and Herschel (André \& Saraceno 2005) Gould Belt surveys. The Spitzer Space Telescope (Spitzer) is an $85 \mathrm{~cm}$ diameter cryogenically cooled satellite telescope designed to operate throughout the infrared regime (Werner et al. 2004b). Its two instruments used in this study are the Infrared Array Camera (IRAC; Fazio et al. 2004), which can observe at 3.6-8.0 $\mu \mathrm{m}$, and the Multiband Imaging Photometer for Spitzer (MIPS; Rieke et al. 2004), which can observe at 24-160 $\mu \mathrm{m}$. Paper I in this series used Spitzer data to identify 200 young stellar object (YSO) candidates toward the
IC 5146 Gould Belt region (Harvey et al. 2008). In this paper, we focus on a different part of the Gould Belt known as the Cepheus Flare region.

The Cepheus Flare is a complex of nebulae that extends $10^{\circ}-20^{\circ}$ out of the plane of the Galactic disk at a Galactic longitude of $\sim 110^{\circ}$ (Hubble 1934). Star formation toward the Flare can be broken down into that associated with the wall of the Local Bubble at $\sim 160 \mathrm{pc}$, that associated with the sweep of the Gould Belt at $\sim 300 \mathrm{pc}$, and that associated with the Perseus arm of the galaxy at $\sim 800 \mathrm{pc}$ (Yonekura et al. 1997; Kiss et al. 2006). Figure 1 shows a visual extinction (Dobashi et al. 2005), CO (Dame et al. 2001), and IRAS $100 \mu \mathrm{m}$ (Miville-Deschênes \& Lagache 2005) finding chart toward the Cepheus Flare. It reveals five associations of dark clouds, each located at the middle distance, including L1148+L1152+L1155, L1172+L1174, L1228, L1241, and L1247+L1251. In this paper, we present IRAC and MIPS data obtained by the SGBS program toward these dark cloud associations and the related dark cloud L1221, which is outside the region shown in Figure 1.

The white contours in Figure 1 show the integrated ${ }^{13} \mathrm{CO}$ emission surrounding the dark cloud associations (Dame et al. 2001). Table 1 lists the cloud positions along with their inferred 


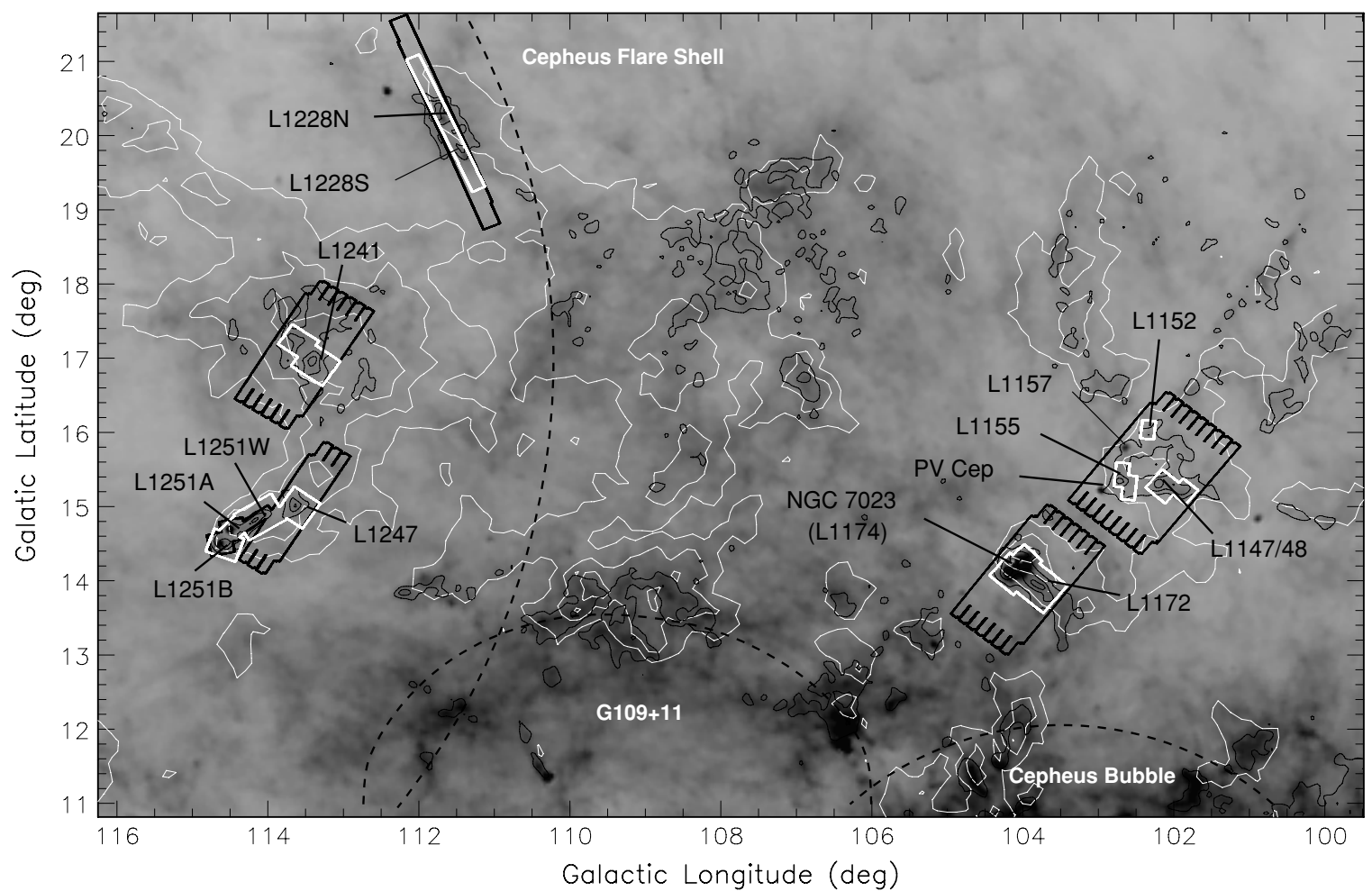

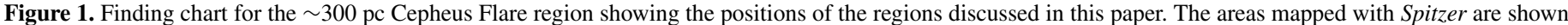

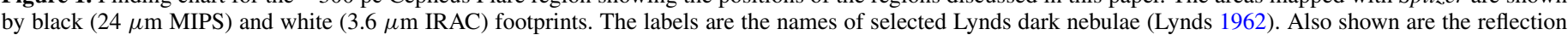

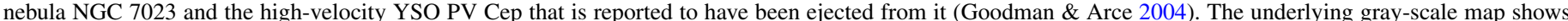

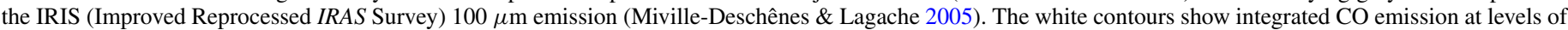

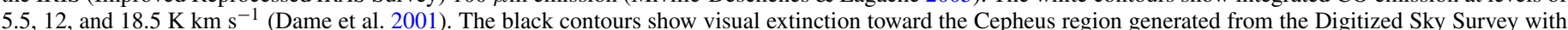

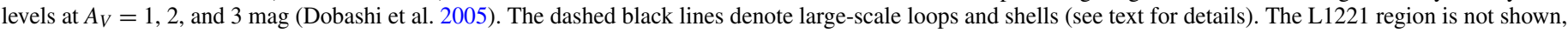
but see Table 1 for its relative position.

Table 1

Dark Cloud Association Properties

\begin{tabular}{lcccccrrr}
\hline \hline \multicolumn{1}{c}{ Association } & $\begin{array}{c}l \\
\left(^{\circ}\right)\end{array}$ & $\begin{array}{c}b \\
\left(^{\circ}\right)\end{array}$ & $\begin{array}{c}\text { R.A. } \\
(2000)\end{array}$ & $\begin{array}{c}\text { Decl. } \\
(2000)\end{array}$ & $\begin{array}{c}\text { Distance } \\
(\mathrm{pc})\end{array}$ & $\begin{array}{r}M_{\text {cloud }} \\
\left(M_{\odot}\right)\end{array}$ & $\begin{array}{c}\Delta V\left({ }^{13} \mathrm{CO}\right) \\
\left(\mathrm{km} \mathrm{s}^{-1}\right)\end{array}$ & $\begin{array}{c}\text { IRAC/MIPS Area } \\
(\mathrm{Sqr} \mathrm{Deg})\end{array}$ \\
\hline L1148+L1152+L1155 & 102.4 & 15.5 & $20^{\mathrm{h}} 40^{\mathrm{m}}$ & $67^{\circ} 40^{\prime}$ & $325 \pm 13$ & 830 & 1.0 & $0.31 / 2.46$ \\
L1172+L1174 & 104.0 & 14.2 & $21^{\mathrm{h}} 02^{\mathrm{m}}$ & $68^{\circ} 10^{\prime}$ & $288 \pm 25$ & 730 & 1.6 & $0.45 / 1.93$ \\
L1221 & 110.7 & 9.73 & $22^{\mathrm{h}} 28^{\mathrm{m}}$ & $69^{\circ} 07^{\prime}$ & $250 \pm 50$ & 40 & 1.5 & $0.05 / 0.40$ \\
L1228 & 111.7 & 20.2 & $20^{\mathrm{h}} 57^{\mathrm{m}}$ & $77^{\circ} 35^{\prime}$ & $200_{-20}^{+100}$ & 230 & 1.6 & $0.44 / 0.77$ \\
L1241 & 113.4 & 16.9 & $22^{\mathrm{h}} 00^{\mathrm{m}}$ & $76^{\circ} 30^{\prime}$ & $300_{-10}^{+50}$ & 1500 & 2.2 & $0.30 / 1.67$ \\
L1247+L1251 & 114.1 & 14.8 & $22^{\mathrm{h}} 30^{\mathrm{m}}$ & $75^{\circ} 10^{\prime}$ & $300_{-10}^{+50}$ & 790 & 1.9 & $0.56 / 1.10$ \\
\hline
\end{tabular}

masses and ${ }^{13} \mathrm{CO}$ line widths from Yonekura et al. (1997). Kun (1998) matched sources in the IRAS point source catalogs with $\mathrm{H} \alpha$ data to identify a catalog of pre-main-sequence stars and dense cores in Cepheus. Their distribution of sources showed that star formation was occurring along the cloud edges. Similarly, Figure 1 shows that regions of high extinction are also distributed toward the edges of the clouds (the lowest $\mathrm{CO}$ contours).

The Cepheus Flare is an expansive and sparsely filled region. Studies have attempted to define structure within the Flare by grouping the scattered objects into associations and groups. Yonekura et al. (1997) mapped Cepheus in CO and identified several large associations of dark clouds. The clouds presented in this paper correspond to Yonekura Group A. Kiss et al. (2006) studied the cloud morphology in Cepheus using their own $256 \mathrm{deg}^{2}$ extinction map. They identified eight cloud complexes across the wider area.

The Cepheus Flare is bounded by a series of shells and loops. Three of these are shown in Figure 1 by dashed lines. The
Cepheus Flare Shell is an expanding supernova bubble at a distance comparable with the clouds in this paper. Its center is located at $l \sim 120^{\circ}, b \sim 17^{\circ}$ (approximately $7^{\circ}$ east of L1241), and it has a radius of $\sim 9.5(50 \mathrm{pc}$ at $300 \mathrm{pc}$ ) (Grenier et al. 1989; Olano et al. 2006). It is possible that the star formation within L1251 has been triggered by the passage of this shell (Olano et al. 2006; Kun et al. 2008). The dark cloud L1228 is coincident with the current radius of the shell (Kun et al. 2008). Other star-formation regions associated with the Cepheus Flare Shell include $\operatorname{L1333}\left(l=129^{\circ}, b=+13^{\circ}\right)$ at a distance of 180 pc (Obayashi et al. 1998). L1333 is in the neighboring constellation of Cassiopeia, on the opposite side of the Cepheus Flare Shell (Kun et al. 2008). The Cepheus Flare Shell and the older, larger Loop III supernova shell appear to be concentric spheres (Kun et al. 2008).

The Cepheus Shell should not be confused with the Cepheus Bubble, which is an expanding dust ring that surrounds the Cep OB2 association (Patel et al. 1998; Ábrahám et al. 2000). Spitzer observations of three clusters associated with Cep OB2 
found that $10 \%$ of the detected disks were "transition" objects (their spectral energy distributions, SEDs, are essentially photospheric except for an infrared excess at the longest wavelengths; Sicilia-Aguilar et al. 2006). Clouds coincident with this bubble are excluded from our analysis as they are believed to be associated with a more distant material (Ábrahám et al. 2000). Other star-formation groups in the direction of Cepheus include S140, Cep OB3, and Cep OB4. These are part of the Yonekura Distant Group, which is at a distance of 600-800 pc (Yonekura et al. 1997). The infrared loop G109+11 was identified by Kiss et al. (2004), who later associated it with "Void \#2" in their extinction survey and a bright rim of excess $12 \mu \mathrm{m}$ emission (Kiss et al. 2006).

In Section 2, we discuss distance estimates of the Cepheus dark cloud associations. In Section 3, we outline the observation strategy, the data reduction procedure, and present the assembled catalog and false-color images of the region. In Section 4, we use several techniques to identify YSO candidates based on their infrared colors. We classify the candidates based on their spectral indices and present color-color diagrams of the resulting list. In Section 5, we analyze the photometry of the YSO candidates, adding additional data where available, to produce SEDs. From these we calculate the Cepheus Flare luminosity function and conduct basic SED modeling. In Section 6, we look at the relation of the YSO candidates to their surroundings and present extinction and MIPS $160 \mu \mathrm{m}$ maps as well as the results of clustering analysis and comparisons with existing surveys. In Section 7, we discuss a star-formation scenario for the Cepheus Flare region. In the Appendix, we discuss each of the individual dark cloud associations and compare results with archival $850 \mu \mathrm{m}$ submillimeter continuum data.

\section{DISTANCE}

The Cepheus Flare contains a number of different components whose distributions on the sky overlap, but whose velocities and distances are different (see Kun et al. 2008 for a review). The dark cloud associations shown in Figure 1 were selected from the Dobashi et al. (2005) extinction map because they each had peak $A_{\mathrm{V}}>3$ and are within a distance of $\sim 500 \mathrm{pc}$. The selected clouds divide into a Galactic West Group comprising the L1172+L1174 and L1148+L1152+L1155 associations at a Galactic longitude of $101^{\circ}-105^{\circ}$ and a Galactic East Group comprising the L1228, L1241, and L1247+L1251 associations at a Galactic longitude of $111^{\circ}-115^{\circ}$. There is a noticeable lack of regions with $A_{\mathrm{V}}>3$ in the span $106^{\circ}-110^{\circ}$ although it does not mean that this span is completely devoid of young stars (Tachihara et al. 2005; Kun et al. 2008) or of molecular material as evidenced by the $\mathrm{CO}$ and extinction maps. However, the lack of $A_{\mathrm{V}}>3$ extinction means that this region falls outside of our selection rules for Spitzer imaging. The arc of the Cepheus Flare Shell would appear to divide the active Galactic East star-formation group and the barren central molecular mass of the Cepheus Flare. Thus, the Cepheus Flare Shell appears to be triggering star formation as its sweeps across the Flare.

The Galactic West Group of dark cloud associations is surrounded by common low-level integrated molecular emission suggesting that they are at a similar distance (e.g., see Figure 1). Many of the distance estimates to these clouds ultimately rely on distance estimates to the Herbig AeBe star HD 200775 embedded within L1174. This is the driving source of the NGC 7023 reflection nebula, but estimates of its distance have been hampered by uncertainties in its spectral type. Whitcomb et al. (1981) surveyed the state of the art at the time of their publication and found a range of distances from 350 to $600 \mathrm{pc}$ before adopting a distance of $440 \mathrm{pc}$. This distance was used by many studies of molecular cores (e.g., Myers et al. 1987; Ward-Thompson et al. 1999). This value is in agreement with the Hipparcos distance of $430_{-90}^{+160}$ pe to HD 200775 (van den Ancker et al. 1997; Bertout et al. 1999).

Estimates of the distance to L1174 based on diagrams of color excess-distance modulus give estimates of $300 \pm 20 \mathrm{pc}$ (Shevchenko et al. 1989) and $288 \pm 25$ pc (Straižys et al. 1992). The second estimate is based on a group of reddened stars in the proximity of the nebula, but does not include the actual distance to HD 200775. For that star, Straižys et al. (1992) derived alternative distances of $275 \mathrm{pc}$ and $417 \mathrm{pc}$ and adopted the former based on the correlation with their first estimate. Straižys et al. (1992) estimated a distance to the L1148/55 molecular ring of $325 \pm 13$ pc. Given the uncertainty of the HD 200775 distance (Kun et al. 2008), we adopt the Straižys et al. distances to L1174 and L1148+L1152+L1155.

The Galactic West Group of dark cloud associations is separated from the Galactic East Group of associations by $\sim 10^{\circ}$. At the adopted distance of these clouds, this is $\sim 50 \mathrm{pc}$. Kun (1998) used Wolf Diagrams to estimate distances of $200_{-20}^{+100} \mathrm{pc}$ for L1228 and $300_{-10}^{+50}$ pc for L1241 and L1247/L1251. The distance to L1251 matches the distance adopted by Lee et al. (2006). Despite their proximity on the sky, L1228 and L1251 are actually on opposite surfaces of the projected Cepheus Flare Shell and thus have different distances (Kun et al. 2008). For L1221, we use the distance adopted by Young et al. (2009). Table 1 lists the adopted distances of the dark cloud associations. The mass of each cloud, $M_{\text {cloud }}$, from Yonekura et al. (1997) has been adjusted to our adopted distances and is listed in Column 7.

\section{OBSERVATIONS AND DATA PROCESSING}

The areas with $A_{\mathrm{V}}>3$ identified from the Dobashi maps were cross-referenced with the Spitzer data archive and the "isolated cores" lists from the c2d program to create two lists of targets. The first list comprised those regions for which public data existed that was compatible with the processing requirements of the $\mathrm{c} 2 \mathrm{~d} / \mathrm{SGBS}$ analysis pipeline, and the second list comprised those regions that had not yet been observed with Spitzer. It was this "second list" that became the basis for Spitzer observations undertaken specifically for the SGBS. Table 2 lists the complete set of Spitzer observations (Astronomical Observation Requests or AORs) included in this paper. The first column gives the name of the central cloud and the second column lists the program identifier (PID). The majority of the archival observations come from the c2d (PID \#139) isolated cores program with the exception of L1228, which was taken as part of the Galactic First Look Survey (PID \#104). The new regions are listed under the SGBS PID \#30574. The extents of the regions listed in Table 2 are shown in Figure 1 by the rectangular footprints (white for IRAC and black for MIPS).

\subsection{Spitzer Gould Belt Data}

Two epochs separated by 5-6 hr were used to take two complete sets of observations of the new SGBS clouds. The redundancy in coverage allowed for the rejection of transient phenomena (including foreground asteroids), the recovery of coverage lost by the blanking of bad pixels, and the full mapping 
Table 2

AORs in the SGBS Cepheus Catalog

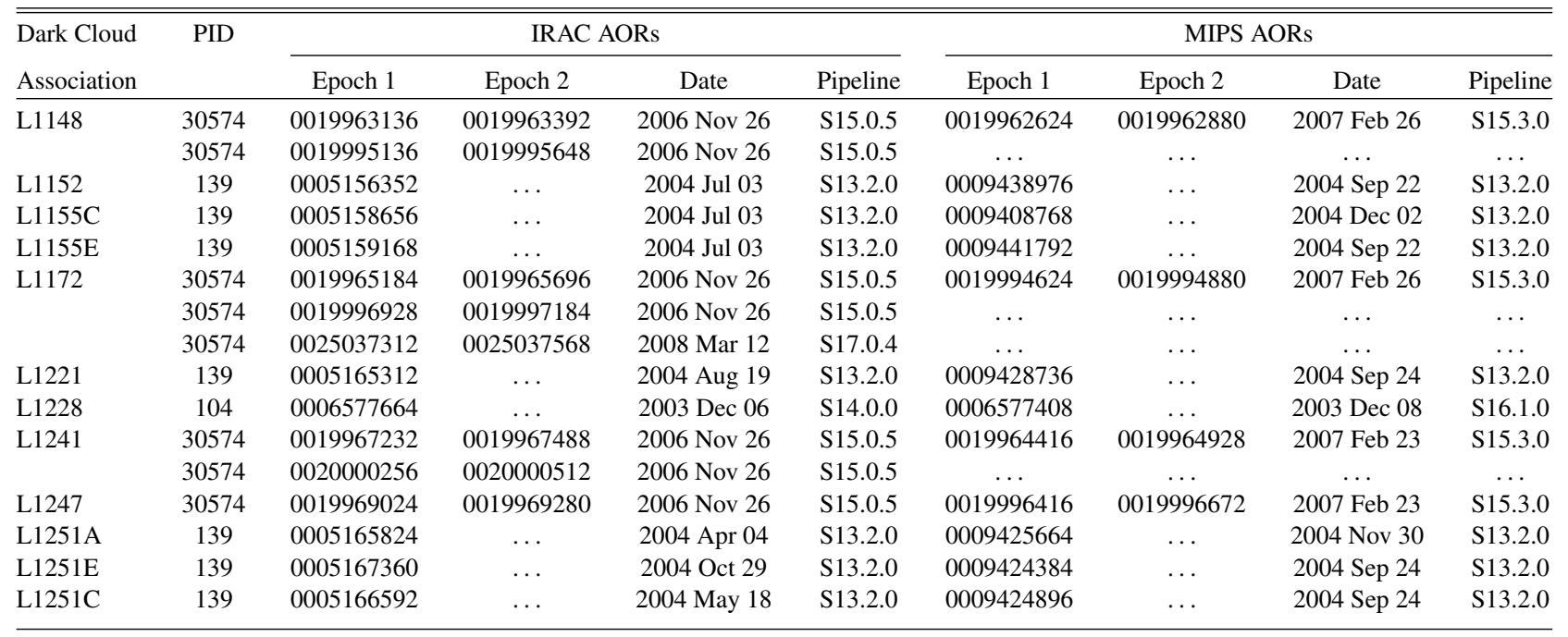

at MIPS $70 \mu \mathrm{m}$ where half the array was not working (each epoch was offset by half the array width). The unique AOR numbers for each epoch's observations are listed in Columns 3 and 4 for IRAC and columns 7 and 8 for MIPS. For the archival observations, only one epoch was obtained. Columns 5 and 9 list the respective dates when the IRAC and MIPS observations were taken. Likewise, Columns 6 and 10 list the version of the Spitzer Science Center (SSC) data pipeline that had been used to process the data prior to their being downloaded from the archive.

The total area mapped was $2.11 \mathrm{deg}^{2}$ with IRAC and $8.33 \mathrm{deg}^{2}$ with MIPS. MIPS mapped a larger area than IRAC because its minimum scan lengths were longer than the diameter of the compact regions of high extinction. The regions for which we have four-band IRAC fluxes are slightly smaller than the areas listed in Table 1. This is because the IRAC detectors are paired into two different pointing offsets resulting in slightly different coverage patterns between 3.6/5.8 $\mu \mathrm{m}$ and $4.5 / 8.0 \mu \mathrm{m}$. The IRAC observations have a total integration time of $48 \mathrm{~s}$ per point split between the two epochs. Each epoch consisted of two dithers. The SGBS MIPS observations were taken with the fast-scanning mode using a 240 arcsec step size and a cross-scan overlap. This gave a total per point integration time of $31.4 \mathrm{~s}$ at 24 and $70 \mu \mathrm{m}$ and $6.1 \mathrm{~s}$ at $160 \mu \mathrm{m}$.

The basic calibrated data (BCD) were downloaded from the Spitzer archive and then ingested into the c2d/SGBS analysis framework (for a full description, see Evans et al. 2007 and Huard et al. 2009, or separately Harvey et al. 2006 for IRAC, and Rebull et al. 2007 for MIPS). In brief, the data were inspected and custom data masks were created to identify bad pixels. The data were then corrected for instrumental effects including bleed over, saturation, and banding effects for IRAC and jailbar and stim flash latents for MIPS. The improved data were mosaiced with the MOPEX package (Makovoz et al. 2006) and source extraction with the c2dphot tool was performed independently at each wavelength and epoch (Evans et al. 2007; Huard et al. 2009). The c2dphot tool is a derivative of the DAOPHOT source extraction and photometry tool (Schechter et al. 1993).

IRAC and MIPS $24 \mu \mathrm{m}$ photometry was performed with c2dphot during source extraction. Each source was characterized by a point-spread function (PSF) fit performed in a 9 pixel $(3.6-5.8 \mu \mathrm{m})$ or 11 pixel $(8 \mu \mathrm{m}$ and $24 \mu \mathrm{m})$ wide box, the local background level was one of the free parameters. Aperture fluxes were measured in a 7 pixel wide box centered on the source and flux uncertainties were estimated from the goodness of the PSF fit. The pixel size of the IRAC mosaics is $1.2 \mathrm{arcsec}$, and the pixel size of the MIPS $24 \mu \mathrm{m}$ mosaics is 2.6 arcsec. The MOPEX point-source fitting package was used for the $70 \mu \mathrm{m}$ photometry (Makovoz et al. 2006). These methods are discussed in detail in the c2d and SGBS delivery documentation (Evans et al. 2007; Huard et al. 2009). The separate epoch/wavelength source lists were band-merged together with the Two Micron All Sky Survey (2MASS) catalog (Skrutskie et al. 2006) and cross-identifications were made at better than 2 arcsec accuracy. Each source was then characterized spectrally and for the quality of detection. The catalog was "band-filled" to produce upper limit flux estimates for sources that were not detected at all wavelengths (Evans et al. 2007; Huard et al. 2009).

Table 3 lists the original survey/instrument, filter, nominal wavelength, flux zero-point $F_{0 \mathrm{pt}}$, and limiting magnitude of the ten bands from the band-merged catalog (2MASS $J-K_{s}$; IRAC 3.6-8.0 $\mu \mathrm{m}$ and MIPS 24-160 $\mu \mathrm{m}$ ) plus additional bands from the second HST Guide Star Catalog (GSC-II, $B-I$ ), the IRAS point-source catalogs $(12-100 \mu \mathrm{m})$, and the Submillimeter Common User Bolometer Array (SCUBA) data archive (450$850 \mu \mathrm{m}$ ). Data for these additional bands were only added for YSO candidates, as described in Section 5.1. The photometric system is based on the Vega magnitude system using the flux zero points taken from the c2d delivery documentation (Evans et al. 2007). These are within 1\%-2\% of the SSC's IRAC $^{11}$ (Reach et al. 2005) and MIPS ${ }^{12}$ zero points. We use the $\mathrm{c} 2 \mathrm{~d}$ zero points to maintain compatibility with the $\mathrm{c} 2 \mathrm{~d}$ delivery documentation (Evans et al. 2007). The minimum uncertainties for individual flux measurements are $4 \%$ and $8 \%$ for IRAC and MIPS (24 $\mu \mathrm{m}$ and $70 \mu \mathrm{m})$, respectively. These uncertainties do not include the zero-point/absolute calibration uncertainties of $1.5 \%$ for IRAC, $4 \%$ for MIPS $24 \mu \mathrm{m}, 20 \%$ for MIPS $70 \mu \mathrm{m}$ (Evans et al. 2007). The absolute Spitzer calibration uncertainties are lower than the individual measurement uncertainties by a factor of 2 or more, except

\footnotetext{
11 http://ssc.spitzer.caltech.edu/irac/calib/

12 http://ssc.spitzer.caltech.edu/mips/calib/
} 
Table 3

Spectral Bands in This Survey

\begin{tabular}{|c|c|c|c|c|c|c|}
\hline Origin & Filter & $\begin{array}{c}\text { Wavelength } \\
(\mu \mathrm{m})\end{array}$ & $\begin{array}{l}F_{0 \mathrm{pt}} \\
(\mathrm{Jy})\end{array}$ & $\begin{array}{c}\text { Limiting } \\
\text { Magnitude }^{\mathrm{a}}\end{array}$ & $\theta^{\mathrm{b}}$ & Ref \\
\hline GSC-II ${ }^{\mathrm{c}}$ & $B$ & 0.44 & 4260.0 & $\ldots$ & 1.7 & 1,8 \\
\hline GSC-II ${ }^{\mathrm{c}}$ & V & 0.55 & 3640.0 & $\ldots$ & $1^{\prime \prime} .7$ & 1,8 \\
\hline GSC-II ${ }^{\mathrm{c}}$ & $R$ & 0.64 & 3080.0 & $\ldots$ & $1^{\prime \prime} .7$ & 1,8 \\
\hline GSC-II ${ }^{\mathrm{c}}$ & $I$ & 0.79 & 2550.0 & $\ldots$ & $1^{\prime \prime} .7$ & 1,8 \\
\hline 2MASS & $J$ & 1.24 & 1594.0 & 16.3 & $2 . .5$ & 2 \\
\hline 2MASS & $H$ & 1.66 & 1024.0 & 15.5 & $2^{\prime \prime} .5$ & 2 \\
\hline 2MASS & $K_{s}$ & 2.16 & 666.7 & 15.3 & $2^{\prime \prime} .5$ & 2 \\
\hline IRAC & IRAC1 & 3.6 & 280.9 & 17.2 & $1^{\prime \prime} .7$ & 3,6 \\
\hline IRAC & IRAC2 & 4.5 & 179.7 & 16.7 & $1^{\prime \prime} .7$ & 3,6 \\
\hline IRAC & IRAC3 & 5.8 & 115.0 & 15.5 & $1^{\prime \prime} .9$ & 3,6 \\
\hline IRAC & IRAC4 & 8.0 & 64.13 & 14.3 & 2.0 & 3,6 \\
\hline$I R A S$ & IRAS1 & 12.0 & $\ldots$ & $\ldots$ & $4^{\prime} .5$ & 4 \\
\hline MIPS & MIPS1 & 24.0 & 7.14 & 10.2 & 6.0 & 3,7 \\
\hline IRAS & IRAS2 & 25.0 & $\ldots$ & $\ldots$ & $4 ! 6$ & 4 \\
\hline IRAS & IRAS3 & 60.0 & $\ldots$ & $\ldots$ & 4.7 & 4 \\
\hline MIPS & MIPS2 & 70.0 & $\ldots$ & $\ldots$ & $18^{\prime \prime}$ & 3,7 \\
\hline IRAS & IRAS4 & 100.0 & $\ldots$ & $\ldots$ & $5^{\prime} .0$ & 4 \\
\hline MIPS & MIPS3 & 160.0 & $\ldots$ & $\ldots$ & $40^{\prime \prime}$ & 3,7 \\
\hline SCUBA & SHORT & 450.0 & $\ldots$ & $\ldots$ & $7^{\prime \prime} .5$ & 5 \\
\hline SCUBA & LONG & 850.0 & $\ldots$ & $\ldots$ & $14^{\prime \prime}$ & 5 \\
\hline
\end{tabular}

Notes.

a Taken as the turnover in the $\mathrm{S} / \mathrm{N}$ limited source count distribution.

b The effective angular resolution at each wavelength. See quoted references for details.

c The GSC-II data have been converted into the Johnson-Cousins photometric system from the natural systems used by its component surveys

References. (1) Bessell 1979; (2) Skrutskie et al. 2006; (3) Evans et al. 2007; (4) Neugebauer et al. 1984; (5) Holland et al. 1999; (6) Fazio et al. 2004; (7) Rieke et al. 2004; (8) Lasker et al. 2008.

for the MIPS $70 \mu \mathrm{m}$ band, which has a significantly higher calibration uncertainty. The limiting magnitudes are identified in Section 3.4 as the turnover in the source count distributions.

\subsection{Archival Spitzer Data}

The c2d data used in this paper come from their final data release $^{13}$ (DR4) and use the same target selection and observation strategy as the SGBS program. A significant difference was that the c2d cores data were taken using the MIPS small map photometry mode rather than the fast-scanning technique. As a result, the $70 \mu \mathrm{m}$ coverage on the c2d cores can actually be smaller than the IRAC area (Evans et al. 2007). No $160 \mu \mathrm{m}$ data were taken for the c2d isolated cores (requesting $160 \mu \mathrm{m}$ data imposes a scheduling limitation as it can only be taken in particular "cold" campaigns). The L1148+L1152+L1155 region contains three c2d cores in close proximity so the entire area was covered by a single SGBS MIPS fast scan to obtain a complete $160 \mu \mathrm{m}$ coverage. The only regions for which $160 \mu \mathrm{m}$ data were not taken were L1221 and L1251.

At the time of writing, the $\mathrm{c} 2 \mathrm{~d}$ studies on the individual first list cores have already been published or are in the process of being published. L1148 was studied by Kauffmann et al. (2005), who discovered a new very low luminosity (VeLLO) Class 0 YSO. L1155C, L1152, and L1228N were studied by Chapman \& Mundy (2009) to examine the extinction law from 3.6 to $24 \mu \mathrm{m}$. Although L1228N was observed by c2d, we use the First Look Galactic data set as it covers both L1228N and L1228S. An analysis of the small YSO group in L1228S was published by Padgett et al. (2004). L1221 was extensively studied by Young et al. (2009), including follow-up observations

\footnotetext{
13 http://ssc.spitzer.caltech.edu/legacy/c2dhistory.html
}

with the VLA. L1251B was studied by Lee et al. (2006), who compared their YSO detections with continuum submillimeter data. This was then followed up by a molecular line study (Lee et al. 2007). The data set was built with the aim of maximizing the coverage with a (relatively) uniform sensitivity. We chose not to add deeper data (e.g., the PID \#3656 data used by Chapman \& Mundy (2009)) as we wish to have all regions covered to approximately the same depth.

The L1152, L1155, and L1221 studies used data from the DR4 c2d data release (Chapman \& Mundy 2009; Young et al. 2009), the same one used by this paper. The L1251 study, however, used data from an earlier data release (DR3) that were processed through version S11 of the SSC pipeline (Lee et al. 2006). Likewise, the First Look study of L1228 used data from version S9.1 of the SSC pipeline (Padgett et al. 2004). The SSC pipeline is constantly being revised to improve calibration and reduce instrumental artifacts. In particular, pipeline versions up to S9.1 used a preliminary flux calibration model. ${ }^{14}$ Improvements in the calibration will change the photometry for regions where a newer pipeline has been used than that in the original study. This is most significant for the L1228 region where it was found that fluxes measured from S14/16 data had increased by an average of $16 \%$ against the $\mathrm{S} 9$ version of the data. The greatest increase was at $70 \mu \mathrm{m}$, which increased by almost $40 \%$.

\subsection{Images of Extended Emission}

Figure 2 shows RGB color composites of $4.5 \mu \mathrm{m}$ (blue), $8.0 \mu \mathrm{m}$ (green), and $24 \mu \mathrm{m}$ (red) toward the regions observed for the SGBS. The green haze that covers a large area of the images comes from the $8.0 \mu \mathrm{m}$ background, which is believed to arise

\footnotetext{
${ }^{14}$ See http://ssc.spitzer.caltech.edu/archanaly/plhistory/ for a revision history.
} 


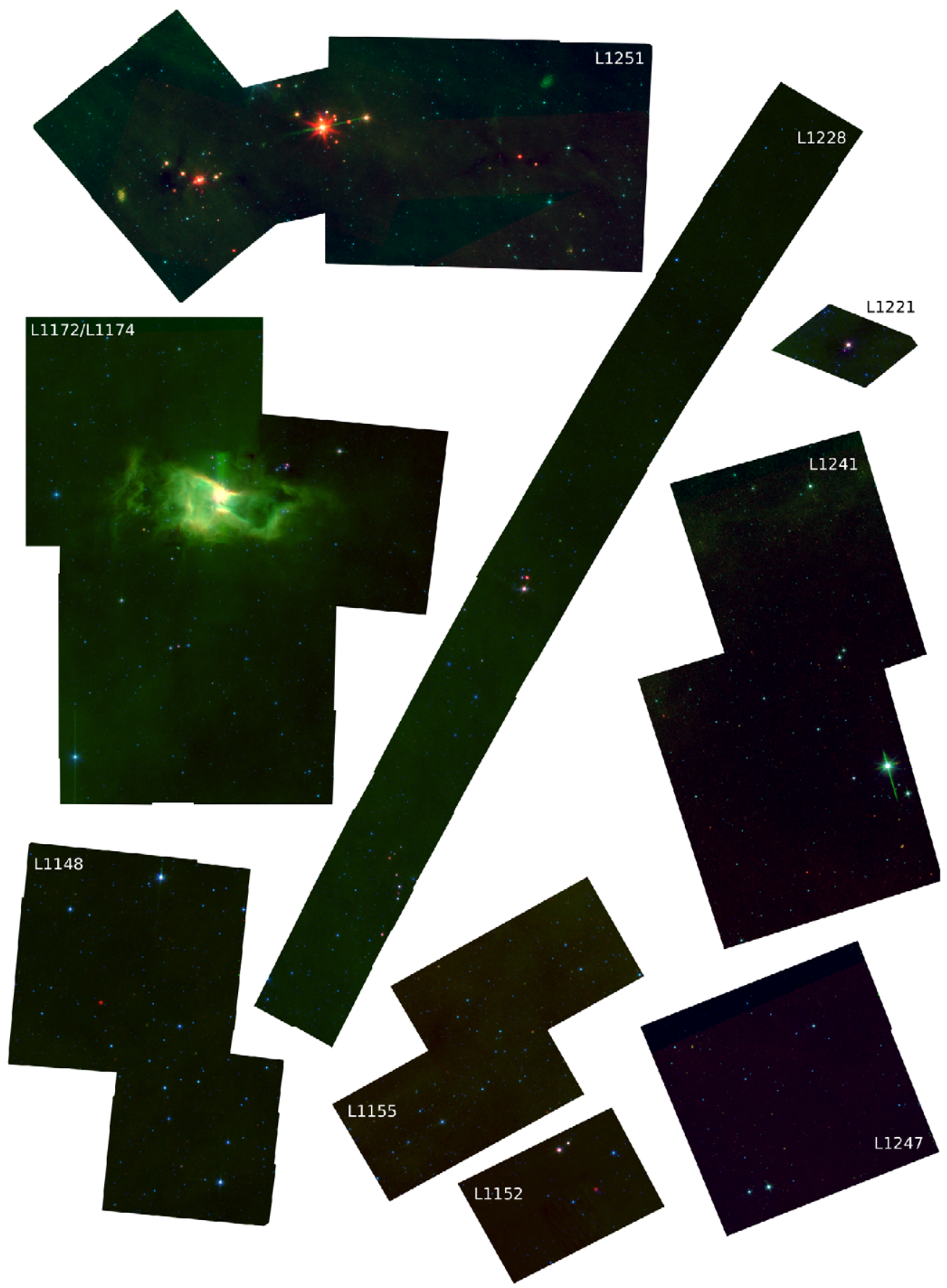

Figure 2. IRAC and MIPS composite RGB images $4.5 \mu \mathrm{m}$ (blue), $8.0 \mu \mathrm{m}$ (green), and $24 \mu \mathrm{m}$ (red) emission toward the regions in this survey. The nebulosity in L1172 is the NGC 7023 reflection nebula. Sources, like some YSOs, that have strong $24 \mu \mathrm{m}$ emission will appear red in this color space. For example, the red source at the center of L1148 is the source discovered by Kauffmann et al. (2005). The gray-scale maps of the individual cores, with astrometry and annotations, are presented in the Appendix.

(A color version of this figure is available in the online journal.)

predominantly from $7.7 \mu \mathrm{m}$ polycyclic aromatic hydrocarbon (PAH) emission (Flagey et al. 2006). In L1241, the haze appears along the edge of the image and is anticorrelated with regions of higher $160 \mu \mathrm{m}$ emission (see Section 6). While the mosaic processing removed a number of instrumental effects, it was unable to correct for the bleeding at $8 \mu \mathrm{m}$ that appears in the color composite images as green smearing upward from the bright center of NGC 7023. The red point source in the L1148 map is the heavily reddened L1148-IRS source studied by Kauffmann et al. (2005).
The brightest extended structure at all wavelengths in this survey is the NGC 7023 reflection nebula. Figure 3 shows three-color composite images covering the visible (Digitized Sky Survey-II; left image), infrared (IRAC; middle image), and far-IR/submillimeter wavelengths (MIPS; right image). The extended cold dust emission, seen in silhouette in the first panel and emission in the last panel, forms a faint cross with HD 200775 at its intersection. The vertical axis of the cross is formed by the north-south filament that includes L1172 and L1174. The east-west axis is formed by the reflection nebula 


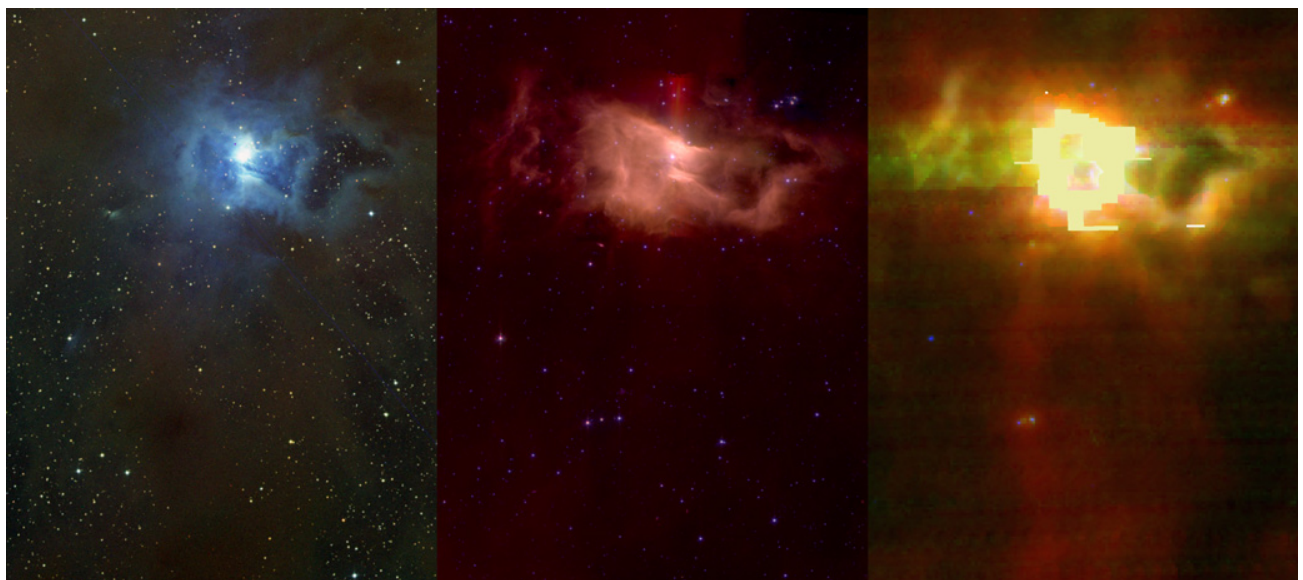

Figure 3. Three composite RGB maps showing how the pattern of emission toward the NGC 7023 region changes between the optical, infrared, and far-infrared regimes. Left: POSS-II $B_{\mathrm{J}}$ (blue), $R_{\mathrm{F}}$ (green), and $I_{\mathrm{N}}$ (red); Middle: IRAC $3.6 \mu \mathrm{m}$ (blue), $5.8 \mu \mathrm{m}$ (green), and $8.0 \mu \mathrm{m}$ (red); Right: MIPS $24 \mu \mathrm{m}$ (blue), $70 \mu \mathrm{m}$ (green), and $160 \mu \mathrm{m}$ (red). The dense gas appears in extinction in the optical image and emission in the far-infrared MIPS image. Embedded YSOs are hidden by the extinction in the optical image, but they appear as bright point sources in the IRAC infrared image.

(A color version of this figure is available in the online journal.)

forming in this filament. The outflow from HD 200775 is currently inactive, but it has generated an asymmetric east-west biconical cavity that is filled with hot atomic gas (Fuente et al. 1998). The larger western lobe of this cavity appears as a hole in the left-hand panel through which background stars are clearly visible. The eastern lobe is truncated by a dense material that is being heated and photodissociated by the YSO (Fuente et al. 1998).

The reflection nebula is confined to the north by a dense filament. This same structure is shown in the strong $3.6 \mu \mathrm{m}$ emission on the leading edge of a colder dense material revealed by the $850 \mu \mathrm{m}$ dust emission (see the Appendix). To the south of NGC 7023 is the L1172D dark nebula that appears in silhouette in the visible image, but reveals three embedded sources in the mid-infrared image. The dust itself emits at the longest wavelengths and shows the bright point source associated with the L1172 SMM 1 embedded protostar. The extended $8 \mu \mathrm{m}$ emission (the red haze in the central image) is anticorrelated, however, with the high visual extinction in the first image and the dense gas in the third image.

\subsection{Source Catalog}

Tables 4 and 5 list the number of objects detected in various combinations of the IRAC or MIPS bands within the SGBS Cepheus Delivery Catalog, which contains a subset of the data included in this paper. Specifically, it includes the five regions: L1148, L1172, L1228, L1241, and L1247+L1251. The other regions (L1155, L1152, and L1221) are available from the SSC as c2d data products. The source detection for SGBS data is defined as an object with a peak intensity $\geqslant 3 \times$ the local $\mathrm{rms}$ in its respective band. A 2 MASS detection constitutes an object seen at $\geqslant 10 \times$ the local rms in both the $H$ and $K_{s}$ bands (a design constraint of the 2MASS catalog itself; see Skrutskie et al. 2006).

Table 4 shows that a total of 71,085 sources were detected in at least one IRAC band, and of those, 6518 were detected in all four bands. Table 5 lists the number of source detections for each of the four IRAC bands and the first two MIPS bands for several different signal-to-noise thresholds. It shows that the majority of these sources were predominantly detected at shorter wavelengths. An important distinction between
Table 4

Detection $(\mathrm{S} / \mathrm{N} \geqslant 3)$ Statistics Across the Delivered Area

\begin{tabular}{|c|c|}
\hline Detected with/in... & Detections \\
\hline$\ldots$ at least one IRAC band & 71085 \\
\hline$\ldots$ all four IRAC bands & 6518 \\
\hline ... three IRAC bands & 6167 \\
\hline ... two IRAC bands & 34848 \\
\hline$\ldots$ only one IRAC band & 23552 \\
\hline$\ldots$ MIPS 1 & 2147 \\
\hline$\ldots$ all four IRAC bands and MIPS 1 & 889 \\
\hline$\ldots$ all four IRAC bands and with 2 MASS $^{\mathrm{a}}$ & 3843 \\
\hline$\ldots$ all four IRAC bands, but not with $2 \mathrm{MASS}^{\mathrm{a}}$ & 2675 \\
\hline$\ldots 2$ MASS alone ${ }^{\mathrm{a}}$ & 1 \\
\hline$\ldots$ IRAC, but not in $2 \mathrm{MASS}^{\mathrm{a}}$ & 67085 \\
\hline \multicolumn{2}{|c|}{ Excluding Extended Sources } \\
\hline$\ldots$ all four IRAC bands & 6183 \\
\hline$\ldots$ all four IRAC bands and with 2 MASS $^{\mathrm{a}}$ & 3824 \\
\hline$\ldots$ IRAC1+2 and $2 \mathrm{MASS}^{\mathrm{a}}$ & 3974 \\
\hline
\end{tabular}

Note. ${ }^{\text {a }}$ A 2 MASS detection is taken as one with $\mathrm{S} / \mathrm{N}>10$ in both $H$ and $K_{s}$.

Table 5

Detection of Sources Above S/N Thresholds for the Entire Mapped Area at Each Wavelength

\begin{tabular}{lcccccc}
\hline \hline Detection & \multicolumn{5}{c}{ Wavelength $(\mu \mathrm{m})$} \\
\cline { 2 - 7 } & 3.6 & 4.5 & 5.8 & 8.0 & 24 & 70 \\
\hline $\mathrm{S} / \mathrm{N}>3$ & 80016 & 61005 & 14674 & 10221 & 9353 & 431 \\
$\mathrm{~S} / \mathrm{N}>5$ & 63148 & 44093 & 8391 & 6172 & 2566 & 384 \\
$\mathrm{~S} / \mathrm{N}>7$ & 48854 & 32061 & 6402 & 4589 & 1027 & 215 \\
$\mathrm{~S} / \mathrm{N}>10$ & 32037 & 19894 & 4270 & 3079 & 390 & 22 \\
$\mathrm{~S} / \mathrm{N}>15$ & 14672 & 9379 & 2082 & 1626 & 1 & 1 \\
\hline
\end{tabular}

Tables 4 and 5 is that the former only refers to the area common to all Spitzer wavelengths, whereas the latter lists statistics for the entire region of the delivery catalog.

Figure 4 shows source count histograms of the number density of sources per magnitude interval per square degree for six bands from the delivery catalog. The gray curve shows all sources. The limiting magnitudes listed in Column 5 of Table 3 are 


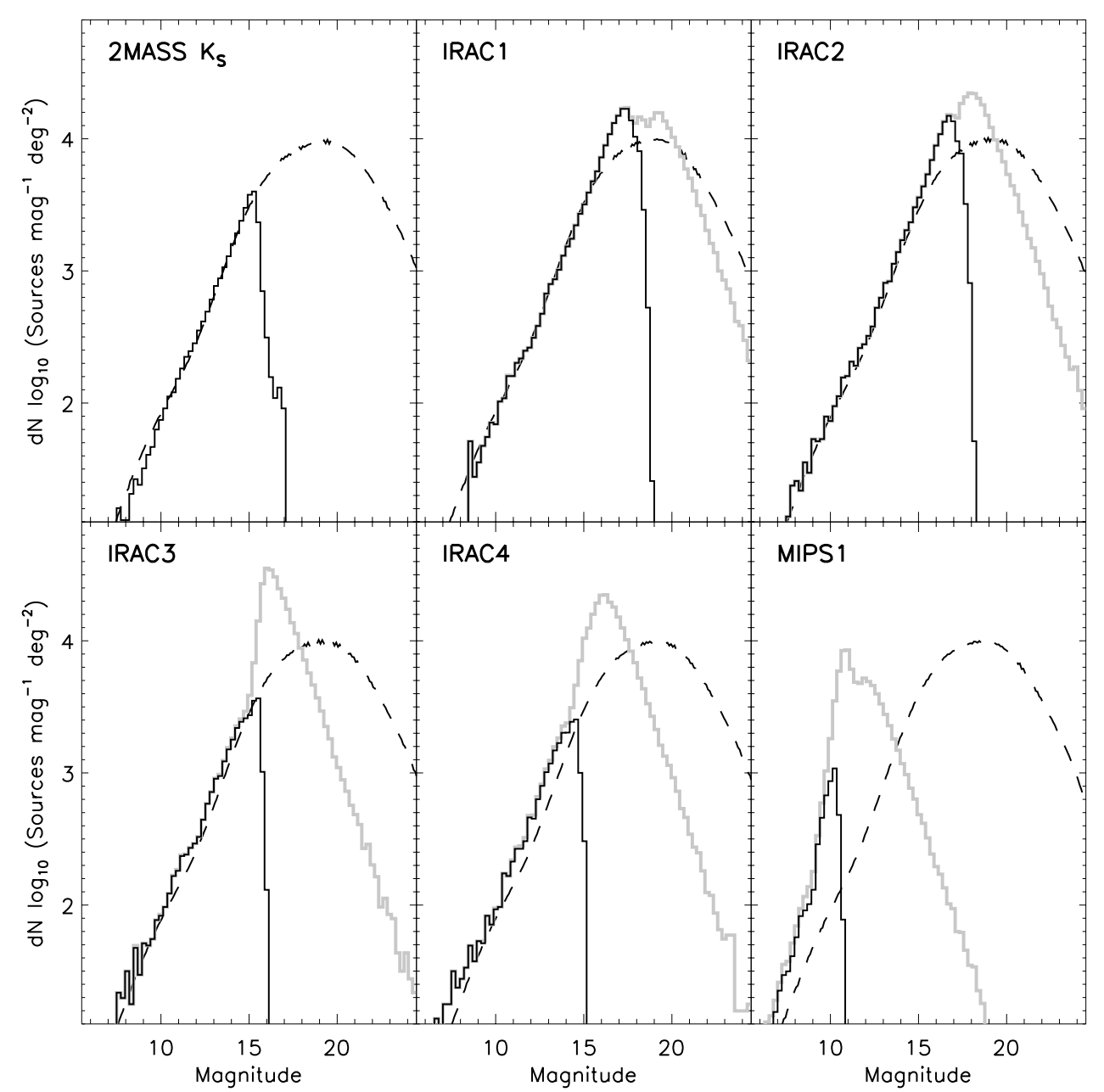

Figure 4. Source counts per square degree per magnitude for six bands from the Cepheus Delivery Catalog. The gray line shows all sources, while the black line shows sources with an $\mathrm{S} / \mathrm{N}>3$. The turn over in each black line is taken as the limiting magnitude for that band. The 2MASS catalog only includes sources with $\mathrm{S} / \mathrm{N}>10$, so no gray line plot is shown for the $K_{s}$ band. The dashed black line is the expected background source count toward Cepheus calculated from the Wainscoat et al. (1992) model of Galactic infrared source counts with a visual extinction of $A_{V}=1$.

taken as the magnitudes where the black curves in Figure 4 turn over. The total source count histogram at $3.6 \mu \mathrm{m}$ is doublepeaked. The $4.5 \mu \mathrm{m}$ source count histogram shows a second peak, but the effect is far less pronounced. Double peaks were also found in the source count histograms for Perseus (Jørgensen et al. 2006) and Chamaeleon II (Porras et al. 2007). Jørgensen et al. (2006) showed that the double peak was not due to the presence of background galaxies by comparing counts of on-cloud and off-cloud sources and that it must derive from something, like extinction, that is common to the entire region. The solid black curves in Figure 4 plot only those sources that meet our detection criteria. The peaks of the black curves for $3.6 \mu \mathrm{m}$ (IRAC1) and $4.5 \mu \mathrm{m}$ (IRAC2) are coincident with the first of the double peaks.

Overplotted in Figure 4 are the source count histograms of Galactic infrared sources toward Cepheus estimated from the model of Wainscoat et al. (1992) updated by J. Carpenter (2001, private communication) to apply to the Spitzer bands. The source counts agree with the model at low magnitudes before diverging at high magnitudes. The divergence from the model increases with increasing wavelength. An excess of sources above the model can be attributed to the detection of extragalactic sources that are not present in the Wainscoat model. The majority of the $24 \mu \mathrm{m}$ (MIPS1) sources are in excess of the Wainscoat model and are therefore probably extragalactic in origin.

\section{CANDIDATE YSOS IN CEPHEUS}

\subsection{Identification of YSO Candidates}

Young stellar objects (YSOs) and background galaxies can have similar infrared colors, but they can be differentiated by the fainter apparent magnitudes of the galaxies. There are several published schemes for identifying YSO candidates from their Spitzer colors, each relying on the specific subsets of the Spitzer wavelength coverage and the equivalent 2MASS fluxes (e.g., Harvey et al. 2006, 2007; Rebull et al. 2007; Allen et al. 2007; Gutermuth et al. 2008). These schemes are commonly calibrated against a known galaxy catalog. SWIRE (the Spitzer Wide-area Infrared Extragalactic Survey, another of the Spitzer legacy programs) was specifically designed to observe the infrared extragalactic background along sightlines that avoided as much foreground (Galactic) contamination as possible (Lonsdale et al. 2003). Thus, it provides an ideal calibration data set to use with any galaxy-rejecting protocol.

IRAC observations were targeted toward regions of high visual extinction with the expectation that these regions will harbor the highest concentration of YSOs. A side effect of the different operational modes of IRAC and MIPS is that the MIPS data cover an area four times as large as the IRAC data. A galaxyrejection scheme that used IRAC-only data would be unusable 
across $3 / 4$ of our surveyed area. Therefore, we adopt different schemes for the common (IRAC+MIPS) and the MIPS-only areas. A significant factor in both schemes is a $24 \mu \mathrm{m}$ MIPS detection. Since that band is not as sensitive as the IRAC bands, a third scheme is adopted for sources detected only with IRAC.

Table 6 lists the YSO candidates identified toward the regions in our survey. The first three sections of Table 6 list the YSO candidates identified by the 5-Band, IRAC-only, and 2MASS/ MIPS schemes, respectively (described below). The first column lists the source number. The second column lists the Spitzer catalog position identifier of each source (a contraction of the source's seven-digit right ascension and declination without delimiters). All positions are given in J2000. Column 3 lists cross-identification names from the literature. Columns 4-9 list the flux and statistical errors or where appropriate the bandfilled upper limit. Column 10 lists a series of flags denoting which candidate identification methods identify the source. The letters F, I, and $\mathrm{M}$ are shown for sources identified by the 5-Band, IRAC, and 2MASS/MIPS methods, respectively. A source may satisfy the rule sets of more than one method, but it is assigned to a group based on the order $F>I>M$. This catalog includes data previously published in a series of $\mathrm{c} 2 \mathrm{~d}$ papers examining individual dark clouds. Column 11 lists references to these and other studies.

\subsubsection{5-Band Identifications}

Harvey et al. (2007) used a 5-Band (4.5, 8.0, and $24 \mu \mathrm{m}$, plus 2MASS $H$ and $K_{S}$ ) scheme to estimate the probability that a given source is a background galaxy. The unnormalized probability $P_{\text {gal }}$ is the product of a series of probabilities that a given source is a background galaxy. Each of these probabilities is based on the selection criteria from a different color-magnitude diagram. The final value of $P_{\text {gal }}$ is moderated by a series of additional factors that include whether the source is extended at $3.6 \mu \mathrm{m}$ or $4.5 \mu \mathrm{m}$ (see Table 1 of Harvey et al. 2007 for a full list). Additionally, the scheme requires that a source be detected at each IRAC band and at MIPS $24 \mu \mathrm{m}$, irrespective of whether that band was actually used to construct $P_{\text {gal }}$.

Under the 5-Band scheme, a catalog is filtered to reject those sources that can be adequately modeled by a stellar photosphere. $P_{\text {gal }}$ is calculated for the remaining sources and these are then filtered to retain sources with a suitably low value of $P_{\text {gal }}$, i.e., rejecting sources that are statistically likely to be background galaxies. From their study of Serpens, Harvey et al. (2007) found that an upper $\operatorname{limit}$ of $\log \left(P_{\text {gal }}\right)<-1.47$ rejected the galaxies from their SWIRE control catalog. This method creates a catalog of YSO candidates that is largely free from background galaxies. The catalog will be luminosity limited partially because it requires [24] $>10$ (fainter objects at [24] are preferentially background galaxies, but this cutoff will also exclude faint YSOs).

Figure 5 shows a histogram of $\log \left(P_{\text {gal }}\right)$ for sources in the Cepheus Catalog with detections in all IRAC bands and MIPS $24 \mu \mathrm{m}$. The peak at -5 contains the majority of the YSO candidates identified by this method. There is also a small tail of sources to the left of the -1.47 divide (the vertical dashed line). There are 12 sources in the tail with $-2.5<\log \left(P_{\text {gal }}\right)<-1.47$ and three $(25 \%)$ of them are previously known YSO candidates. We choose to retain the canonical dividing line for YSO candidates for consistency with the c2d studies (Harvey et al. 2007; Evans et al. 2009) and because moving the division by any significant amount would exclude the three known sources.

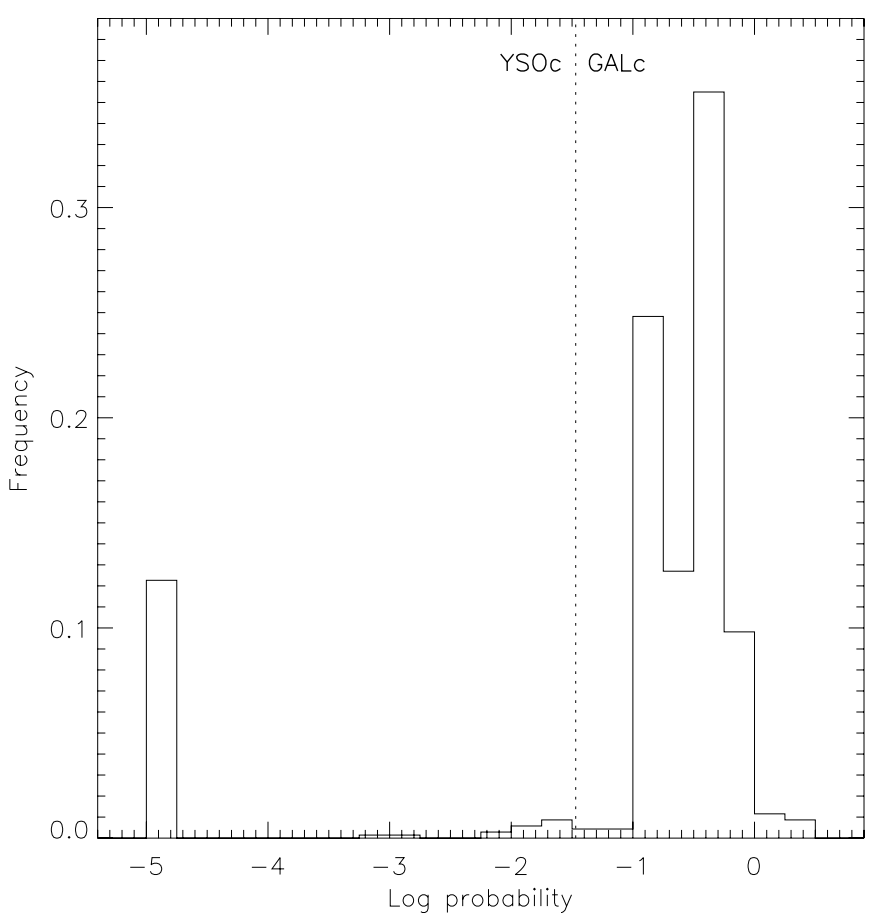

Figure 5. Histogram of $\log \left(P_{\text {gal }}\right)$ for the sources in the Cepheus catalog that were detected in each IRAC band and MIPS $24 \mu \mathrm{m}$. The dashed line shows the $\log \left(P_{\text {gal }}\right)=-1.47$ criterion established by Harvey et al. (2007) as the divide between YSO candidates (YSOc) and galaxy candidates (GALc). The majority of the galaxy candidates appear as a distribution at $-1<\log \left(P_{\text {gal }}\right)<0$, whereas the majority of the YSO candidates appear as a separate peak at $\log \left(P_{\text {gal }}\right)=-5$.

If half of the tail sources were background galaxies, it would represent a contamination of $\sim 5 \%$ in the total number of YSO candidates identified by the 5-Band method.

Figure 6 shows a sequence of color-color plots for sources detected in all four IRAC bands and shows the cutoffs Harvey et al. (2007) used to construct $P_{\text {gal }}$. Dashed lines show "fuzzy" limits, while solid lines show hard limits. The left panel in each pair shows the SGBS data. Black filled circles and crosses are, respectively, the point-like and extended sources identified as YSO candidates by $P_{\text {gal }}$, while dark gray circles are sources identified as point-like (filled circles) and extended (open circles) galaxies. Also shown by pale gray points are sources that were identified as stellar photospheres via their SEDs. The right-hand panel of each pair of plots in Figure 6 shows a contour plot of the SWIRE Catalog after it has been processed in a manner similar to the SGBS Catalog. It can be seen how the limits used in the calculation of $P_{\text {gal }}$ have been chosen to reject regions of parameter space where there are significant numbers of SWIRE galaxies. These plots show that the galaxies are tightly clustered and that there is no significant overlap between the different candidate types. The 5-Band scheme identified 98 YSO candidates and one possible YSO candidate with a bandfilled (upper limit) flux at $8 \mu \mathrm{m}$ (shown as an open square in Figure 6). The sources that satisfy the 5-Band scheme are listed with an F flag in Column 10 of Table 6.

\subsubsection{IRAC-only Identifications}

For those sources that have no detection at $24 \mu \mathrm{m}$, we must use the selection rules based on IRAC colors alone. For this, we use the selection rules from Harvey et al. (2008). They define $([4.5]-[8.0]<0.5$ and $[8.0]>13-([4.5]-[8.0]))$ to reject galaxies and stars. The upper row of Figure 7 shows a plot of 
Table 6

Cepheus YSO Candidates

\begin{tabular}{|c|c|c|c|c|c|c|c|c|c|c|}
\hline Id. & $\begin{array}{l}\text { Name/Position } \\
\text { (SSTgbsJ) }\end{array}$ & Literature Name $^{\mathrm{a}}$ & $\begin{array}{c}3.6(\mu \mathrm{m}) \\
(\mathrm{mJy})\end{array}$ & $\begin{array}{c}4.5(\mu \mathrm{m}) \\
(\mathrm{mJy})\end{array}$ & $\begin{array}{c}5.8(\mu \mathrm{m}) \\
(\mathrm{mJy})\end{array}$ & $\begin{array}{c}8.0(\mu \mathrm{m}) \\
(\mathrm{mJy})\end{array}$ & $\begin{array}{l}24.0(\mu \mathrm{m}) \\
(\mathrm{mJy})\end{array}$ & $\begin{array}{c}70.0(\mu \mathrm{m}) \\
(\mathrm{mJy})\end{array}$ & Flags $^{b}$ & Ref. \\
\hline \multicolumn{11}{|c|}{ 1. 5-Band YSO Candidates } \\
\hline 1 & $2035463+6753020$ & L1152 1; [K98b] 4 & $3.53 \pm 0.19$ & $6.30 \pm 0.33$ & $5.45 \pm 0.27$ & $3.31 \pm 0.16$ & 273. \pm 25 & $3670 . \pm 341$ & $\mathrm{~F}$ & $1 ; 8$ \\
\hline 2 & $2036116+6757093$ & L1152 2 & $41.3 \pm 2.0$ & $47.9 \pm 2.3$ & $56.2 \pm 2.7$ & $74.5 \pm 3.6$ & 173. \pm 16 & 347. \pm 33 & FIM & 1 \\
\hline 3 & $2036198+6756316$ & L1152 3; [K98b] 6; [K98c] EM* 6; GM 3-12 & $228 . \pm 12$ & 243. \pm 16 & 215. \pm 10 & 262. \pm 13 & 634. \pm 59 & $1080 . \pm 102$ & FIM & $1 ; 8 ; 10 ; 15$ \\
\hline 4 & $2039430+6708301$ & & $2.39 \pm 0.12$ & $2.93 \pm 0.14$ & $3.46 \pm 0.18$ & $4.39 \pm 0.21$ & $9.20 \pm 0.87$ & $\ldots$ & FI & \\
\hline 5 & $2040567+6723050$ & L1148-IRS; [K98b] 8 & $1.32 \pm 0.07$ & $1.31 \pm 0.06$ & $0.797 \pm 0.049$ & $1.16 \pm 0.06$ & $75.3 \pm 7.0$ & 331. \pm 33 & FI & $2 ; 8$ \\
\hline 6 & $2055371+7738196$ & L1228 4 & $5.96 \pm 0.29$ & $5.13 \pm 0.25$ & $4.25 \pm 0.22$ & $4.21 \pm 0.20$ & $4.05 \pm 0.39$ & & FIM & 1 \\
\hline 7 & $2057067+7736561$ & L1228 5; HH 200 IRS & $6.87 \pm 0.38$ & $9.72 \pm 0.48$ & $12.5 \pm 0.6$ & $16.3 \pm 0.8$ & $269 . \pm 25$ & $2060 . \pm 236$ & FIM & $1 ; 15$ \\
\hline 8 & $2057079+7736597$ & L1228 6 & $3.03 \pm 0.15$ & $3.35 \pm 0.17$ & $3.59 \pm 0.18$ & $8.53 \pm 0.41$ & 153. \pm 14 & & FIm & 1 \\
\hline 9 & $2057130+7735437$ & L1228 7; [K98b] 12; [RR96] HH 1991 & $260 . \pm 13$ & 406. \pm 23 & 513. \pm 24 & 742. \pm 36 & $1860 . \pm 181$ & $9000 . \pm 983$ & FIM & $1 ; 8 ; 11$ \\
\hline 10 & $2057155+7734236$ & L1228 8 & $2.39 \pm 0.12$ & $2.52 \pm 0.12$ & $2.62 \pm 0.13$ & $2.83 \pm 0.14$ & $4.70 \pm 0.45$ & $\ldots$ & FIm & 1 \\
\hline 11 & $2057170+7736586$ & L1228 9 & $39.3 \pm 1.9$ & $42.5 \pm 2.1$ & $39.1 \pm 1.9$ & $41.1 \pm 1.9$ & $77.1 \pm 7.1$ & $\ldots$ & FIM & 1 \\
\hline 12 & $2058400+7727454$ & L1228 10 & $20.2 \pm 1.0$ & $23.5 \pm 1.1$ & $23.6 \pm 1.1$ & $24.3 \pm 1.2$ & $41.2 \pm 3.8$ & $\ldots$ & FIM & 1 \\
\hline 13 & $2059154+7733229$ & & $25.1 \pm 1.2$ & $19.5 \pm 0.9$ & $17.1 \pm 0.8$ & $20.0 \pm 0.9$ & $22.8 \pm 2.1$ & $\ldots$ & FIM & \\
\hline 14 & $2059175+7728579$ & & $19.2 \pm 0.9$ & $17.0 \pm 0.8$ & $14.3 \pm 0.7$ & $13.3 \pm 0.6$ & $13.7 \pm 1.3$ & & FIM & \\
\hline 15 & $2059228+6814438$ & V* FT Cep; [K98b] 14; [K98c] EM* 26 & 348. \pm 18 & 368. \pm 19 . & $365 . \pm 18$ & $500 . \pm 24$ & 919. \pm 86 & $600 . \pm 62$ & FIM & $8 ; 15$ \\
\hline 16 & $2100203+6808270$ & NGC 7023 S 41 & $41.4 \pm 2.0$ & $44.0 \pm 2.1$ & $44.2 \pm 2.1$ & $47.1 \pm 2.2$ & $119 . \pm 11$ & 713. \pm 143 & FIM & 7 \\
\hline 17 & $2100207+6813169$ & & $3.52 \pm 0.19$ & $13.1 \pm 0.7$ & $21.3 \pm 1.0$ & $25.5 \pm 1.2$ & $602 . \pm 56$ & $3280 . \pm 391$ & FI & \\
\hline 18 & $2100221+6812585$ & & $9.24 \pm 0.53$ & $31.5 \pm 1.6$ & $47.4 \pm 2.2$ & $59.8 \pm 2.8$ & 142. \pm 13 & $\ldots$ & FI & \\
\hline 19 & $2100289+6813023$ & & $21.9 \pm 1.1$ & $22.5 \pm 1.1$ & $21.5 \pm 1.0$ & $23.6 \pm 1.1$ & $23.1 \pm 2.2$ & $\ldots$ & FIM & \\
\hline 20 & $2100321+6812475$ & & 125. \pm 6 & $108 . \pm 5$ & $96.7 \pm 4.6$ & $89.1 \pm 4.3$ & $69.0 \pm 6.4$ & 293. \pm 72 & FIM & \\
\hline 21 & $2100366+6803446$ & & $19.6 \pm 1.0$ & $12.5 \pm 0.6$ & $8.58 \pm 0.41$ & $5.38 \pm 0.25$ & $1.80 \pm 0.29$ & $\ldots$ & $\mathrm{F}$ & \\
\hline 22 & $2100380+7706598$ & & $3.13 \pm 0.15$ & $2.24 \pm 0.11$ & $1.60 \pm 0.09$ & $0.961 \pm 0.052$ & $3.68 \pm 0.38$ & $\ldots$ & $\mathrm{FM}$ & \\
\hline 23 & $2100555+6811273$ & & $8.94 \pm 0.43$ & $7.80 \pm 0.37$ & $6.97 \pm 0.34$ & $8.37 \pm 0.40$ & $9.70 \pm 0.95$ & $\ldots$ & FIM & \\
\hline 24 & $2100581+6809383$ & NGC 7023 S 36 & $17.1 \pm 0.8$ & $14.6 \pm 0.7$ & $13.8 \pm 0.7$ & $17.1 \pm 0.8$ & $20.9 \pm 2.0$ & $\ldots$ & FIM & 7 \\
\hline 25 & $2101037+6813093$ & & $24.0 \pm 1.2$ & $22.4 \pm 1.1$ & $19.4 \pm 0.9$ & $20.6 \pm 1.0$ & $47.6 \pm 4.5$ & $\ldots$ & FIM & \\
\hline 26 & $2101087+6812526$ & & $3.74 \pm 0.18$ & $3.54 \pm 0.17$ & $3.42 \pm 0.17$ & $3.80 \pm 0.24$ & $5.25 \pm 0.61$ & $\ldots$ & FIM & \\
\hline 27 & $2101264+6810385$ & NGC 7023 S J & $59.1 \pm 3.2$ & $90.1 \pm 4.4$ & $81.5 \pm 4.0$ & 146. \pm 10 & $221 . \pm 28$ & $\ldots$ & FIM & $7 ; 15$ \\
\hline 28 & $2101264+7707028$ & L1228 S1(a) & $4.46 \pm 0.22$ & $4.92 \pm 0.23$ & $4.84 \pm 0.24$ & $5.51 \pm 0.26$ & $21.5 \pm 2.0$ & $\ldots$ & FIm & $3 ; 15$ \\
\hline 29 & $2101273+6811384$ & & $16.5 \pm 0.8$ & $16.5 \pm 0.8$ & $18.1 \pm 0.9$ & $21.9 \pm 1.0$ & $31.4 \pm 3.0$ & $\ldots$ & FIM & \\
\hline 30 & $2101293+7702373$ & L1228 S6 & 167. \pm 9 & $115 . \pm 7$ & $92.0 \pm 5.0$ & $97.5 \pm 5.0$ & 175. \pm 16 & $145 . \pm 17$ & FIM & $3 ; 15$ \\
\hline 31 & $2101297+6813468$ & & $18.7 \pm 0.9$ & $17.6 \pm 0.9$ & $17.9 \pm 0.8$ & $18.4 \pm 0.9$ & $45.6 \pm 4.2$ & & FIM & \\
\hline 32 & $2101310+7701535$ & L1228 S7; [K98c] EM* 40 & $18.8 \pm 0.9$ & $13.2 \pm 0.6$ & $8.97 \pm 0.43$ & $5.81 \pm 0.28$ & $53.6 \pm 5.0$ & $65.5 \pm 7.7$ & FM & $3 ; 8 ; 15$ \\
\hline 33 & $2101326+7701175$ & L1228 S8 & $52.0 \pm 2.9$ & $53.3 \pm 2.8$ & $50.1 \pm 2.5$ & $63.8 \pm 3.0$ & $78.2 \pm 7.3$ & $74.3 \pm 9.8$ & FIM & $3 ; 15$ \\
\hline 34 & $2101328+6811202$ & & $3.07 \pm 0.16$ & $8.78 \pm 0.46$ & $14.8 \pm 0.7$ & $23.3 \pm 1.3$ & 180. \pm 19 & & FI & \\
\hline 35 & $2101351+7703567$ & L1228 S4 & $22.0 \pm 1.1$ & $22.6 \pm 1.1$ & $21.0 \pm 1.0$ & $22.1 \pm 1.0$ & $91.3 \pm 8.5$ & 406. \pm 48 & FIM & $3 ; 15$ \\
\hline
\end{tabular}


Table 6

(Continued)

\begin{tabular}{|c|c|c|c|c|c|c|c|c|c|c|}
\hline Id. & $\begin{array}{c}\text { Name/Position } \\
\text { (SSTgbsJ) }\end{array}$ & Literature Name $^{\mathrm{a}}$ & $\begin{array}{c}3.6(\mu \mathrm{m}) \\
(\mathrm{mJy})\end{array}$ & $\begin{array}{c}4.5(\mu \mathrm{m}) \\
(\mathrm{mJy})\end{array}$ & $\begin{array}{c}5.8(\mu \mathrm{m}) \\
(\mathrm{mJy})\end{array}$ & $\begin{array}{c}8.0(\mu \mathrm{m}) \\
(\mathrm{mJy})\end{array}$ & $\begin{array}{c}24.0(\mu \mathrm{m}) \\
(\mathrm{mJy})\end{array}$ & $\begin{array}{c}70.0(\mu \mathrm{m}) \\
(\mathrm{mJy})\end{array}$ & Flags ${ }^{\mathrm{b}}$ & $\begin{array}{l}\text { Ref. } \\
\text {. }\end{array}$ \\
\hline 36 & $2101358+6813257$ & & $62.1 \pm 3.1$ & $66.5 \pm 3.4$ & $44.3 \pm 2.1$ & $61.9 \pm 3.0$ & $69.3 \pm 6.5$ & & FIM & \\
\hline 37 & $2101395+7706165$ & L1228 S2 & $4.77 \pm 0.23$ & $4.72 \pm 0.23$ & $4.38 \pm 0.22$ & $5.05 \pm 0.24$ & $22.5 \pm 2.1$ & $63.7 \pm 8.6$ & FIM & $3 ; 15$ \\
\hline 38 & $2101425+6812571$ & NGC 7023 S N & $35.3 \pm 1.8$ & $29.4 \pm 1.6$ & $26.6 \pm 1.3$ & $28.3 \pm 1.4$ & $52.4 \pm 4.9$ & $\ldots$ & FIM & $7 ; 15$ \\
\hline 39 & $2101437+6750269$ & & $21.3 \pm 1.0$ & $18.1 \pm 0.9$ & $14.4 \pm 0.7$ & $14.3 \pm 0.7$ & $20.7 \pm 1.9$ & $\ldots$ & FIM & \\
\hline 40 & $2101439+6814033$ & & $31.6 \pm 1.5$ & $43.3 \pm 2.2$ & $51.3 \pm 2.4$ & $46.9 \pm 2.2$ & $286 . \pm 27$ & 266. \pm 36 & FIM & \\
\hline 41 & $2101454+6804213$ & & $13.5 \pm 0.7$ & $12.8 \pm 0.7$ & $9.49 \pm 0.49$ & $17.9 \pm 0.9$ & $10.3 \pm 1.0$ & $\ldots$ & FIM & \\
\hline 42 & $2101467+6808453$ & V* FU Cep; NGC 7023 S C & $42.1 \pm 2.0$ & $36.0 \pm 1.7$ & $25.3 \pm 1.2$ & $20.1 \pm 1.0$ & $35.4 \pm 6.0$ & $\ldots$ & FM & $7 ; 15$ \\
\hline 43 & $2101496+7705479$ & L1228 S3 & $18.3 \pm 0.9$ & $13.8 \pm 0.7$ & $12.4 \pm 0.6$ & $21.5 \pm 1.0$ & 133. \pm 12 & $350 . \pm 35$ & FIM & $3 ; 15$ \\
\hline 44 & $2101527+6809519$ & NGC 7023 S 44 & $10.6 \pm 0.5$ & $13.4 \pm 0.7$ & $14.6 \pm 0.8$ & $20.4 \pm 1.2$ & $25.1 \pm 3.9$ & $\ldots$ & FIM & 7 \\
\hline 45 & $2102025+6811587$ & & $1.81 \pm 0.09$ & $2.73 \pm 0.13$ & $2.95 \pm 0.21$ & $<6.79$ & $32.2 \pm 5.7$ & $\ldots$ & FM & \\
\hline 46 & $2102050+7657185$ & L1228 S9; [K98c] EM* 43 & $52.0 \pm 2.5$ & $40.1 \pm 1.9$ & $31.4 \pm 1.5$ & $39.8 \pm 1.9$ & $219 . \pm 20$ & 294. \pm 29 & FIM & $3 ; 8 ; 15$ \\
\hline 47 & $2102140+6813595$ & & $12.6 \pm 0.6$ & $11.2 \pm 0.6$ & $9.75 \pm 0.49$ & $10.2 \pm 0.5$ & $12.4 \pm 1.2$ & $\ldots$ & FIM & \\
\hline 48 & $2102141+6807308$ & & $7.76 \pm 0.40$ & $12.0 \pm 0.6$ & $15.4 \pm 0.8$ & $27.1 \pm 1.6$ & 107. \pm 10 & $\ldots$ & FIm & \\
\hline 49 & $2102212+6754203$ & L1172 SMM 1 & $13.8 \pm 0.7$ & $32.3 \pm 1.6$ & $43.1 \pm 2.1$ & $52.0 \pm 2.5$ & $252 . \pm 23$ & $705 . \pm 72$ & FI & 6 \\
\hline 50 & $2102273+6754186$ & L1172 SMM 2 & $1.03 \pm 0.06$ & $1.15 \pm 0.07$ & $0.659 \pm 0.049$ & $0.356 \pm 0.033$ & $5.63 \pm 0.64$ & $\ldots$ & $\mathrm{F}$ & 6 \\
\hline 51 & $2102280+6808477$ & & $4.91 \pm 0.24$ & $4.87 \pm 0.24$ & $4.84 \pm 0.24$ & $6.94 \pm 0.33$ & $21.4 \pm 2.0$ & $\ldots$ & FIM & \\
\hline 52 & $2102282+6803298$ & & $51.1 \pm 2.6$ & $45.1 \pm 2.2$ & $44.7 \pm 2.7$ & $64.4 \pm 3.2$ & $92.7 \pm 8.6$ & $88.0 \pm 12.3$ & FI & 15 \\
\hline 53 & $2102299+6754083$ & & $100.0 \pm 5.0$ & $120 . \pm 6$ & 127. \pm 6 & $130 . \pm 7$ & 166. \pm 15 & $249 . \pm 35$ & FIM & \\
\hline 54 & $2102324+7655100$ & & $3.55 \pm 0.17$ & $2.96 \pm 0.14$ & $2.48 \pm 0.13$ & $2.14 \pm 0.11$ & $1.28 \pm 0.19$ & $\ldots$ & FIm & \\
\hline 55 & $2102330+6807292$ & $V^{*}$ FW Cep & $40.1 \pm 2.0$ & $34.8 \pm 1.7$ & $30.6 \pm 1.5$ & $29.4 \pm 1.4$ & $14.6 \pm 1.4$ & $\ldots$ & FIM & 15 \\
\hline 56 & $2102549+6806210$ & & $14.9 \pm 0.7$ & $14.4 \pm 0.7$ & $12.1 \pm 0.6$ & $11.9 \pm 0.6$ & $31.5 \pm 2.9$ & $\ldots$ & FIM & \\
\hline 57 & $2102595+6806322$ & [K98b] 30; GN 21.02.4.02 & $13.8 \pm 0.7$ & $18.5 \pm 0.9$ & $27.1 \pm 1.3$ & $78.6 \pm 3.9$ & $239 . \pm 22$ & $87.5 \pm 19.0$ & FIM & $8 ; 15$ \\
\hline 58 & $2102596+6808119$ & & $14.1 \pm 0.7$ & $10.1 \pm 0.5$ & $6.87 \pm 0.33$ & $4.24 \pm 0.22$ & $1.74 \pm 0.24$ & $\ldots$ & Fm & \\
\hline 59 & $2103076+6808340$ & & $27.2 \pm 1.3$ & $23.9 \pm 1.1$ & $20.5 \pm 1.0$ & $22.6 \pm 1.1$ & $32.4 \pm 3.0$ & $\ldots$ & FIM & \\
\hline 60 & $2103244+6759067$ & V* EH Cep; [K98b] 33; [K98c] EM* 42 & $310 . \pm 15$ & 288. \pm 20 & $280 . \pm 14$ & 364. \pm 18 & 435. \pm 40 & $97.4 \pm 11.2$ & FIM & $8 ; 15$ \\
\hline 61 & $2103594+6749296$ & & $15.9 \pm 0.8$ & $14.6 \pm 0.7$ & $12.5 \pm 0.6$ & $12.4 \pm 0.6$ & $18.5 \pm 1.7$ & $\ldots$ & FIM & \\
\hline 62 & $2104156+6742464$ & & $1020 . \pm 167$ & $2850 . \pm 157$ & $2780 . \pm 182$ & $1780 . \pm 100$ & 366. \pm 34 & $\ldots$ & FI & \\
\hline 63 & $2228030+6901166$ & L1221 IRS 1 & 109. \pm 7 & $149 . \pm 11$ & 392. \pm 20 . & 387. \pm 26 & 1940. \pm 187 & $6940 . \pm 641$ & FIM & 4 \\
\hline 64 & $2228074+6900389$ & L1221 IRS 3; [RR98] HH 363 VLA 4 & $0.567 \pm 0.033$ & $3.10 \pm 0.18$ & $4.96 \pm 0.25$ & $3.84 \pm 0.19$ & $47.5 \pm 4.4$ & $5080 . \pm 469$ & FI & $4 ; 13$ \\
\hline 65 & $2229333+7513160$ & & $5.62 \pm 0.28$ & $10.5 \pm 0.5$ & $15.6 \pm 0.8$ & $18.2 \pm 0.9$ & $57.1 \pm 5.3$ & $150 . \pm 15$ & FI & \\
\hline 66 & $2229594+7514037$ & [K93c] 4; [TW96] T3 & $15.8 \pm 0.9$ & $21.8 \pm 1.2$ & $25.2 \pm 1.3$ & $27.3 \pm 1.4$ & 277. \pm 26 & 848. \pm 80 & FIM & $9 ; 12$ \\
\hline 67 & $2230318+7514094$ & [TW96] T2 & $0.134 \pm 0.017$ & $0.421 \pm 0.042$ & $0.315 \pm 0.040$ & $0.162 \pm 0.033$ & $5.10 \pm 0.49$ & $1400 . \pm 131$ & $\mathrm{~F}$ & 12 \\
\hline 68 & $2231056+7513372$ & [K93c] 6; [TW96] T1a & $0.430 \pm 0.029$ & $1.07 \pm 0.08$ & $0.753 \pm 0.049$ & $0.372 \pm 0.032$ & $1.63 \pm 0.24$ & 688. \pm 65 & $\mathrm{~F}$ & $9 ; 12$ \\
\hline 69 & $2234405+7517442$ & & $30.2 \pm 1.5$ & $28.4 \pm 1.4$ & $27.7 \pm 1.4$ & $35.6 \pm 1.8$ & 141. \pm 13 & 257. \pm 24 & FIM & \\
\hline 70 & $2234483+7513352$ & & $8.97 \pm 0.44$ & $7.30 \pm 0.35$ & $6.68 \pm 0.32$ & $9.04 \pm 0.43$ & $18.2 \pm 1.7$ & $50.9 \pm 5.8$ & FIM & \\
\hline
\end{tabular}




\begin{tabular}{|c|c|c|c|c|c|c|c|c|c|c|}
\hline Id. & $\begin{array}{c}\text { Name/Position } \\
(\text { SSTgbsJ) }\end{array}$ & Literature Name $^{\mathrm{a}}$ & $\begin{array}{c}3.6(\mu \mathrm{m}) \\
(\mathrm{mJy})\end{array}$ & $\begin{array}{c}4.5(\mu \mathrm{m}) \\
(\mathrm{mJy})\end{array}$ & $\begin{array}{c}5.8(\mu \mathrm{m}) \\
(\mathrm{mJy})\end{array}$ & $\begin{array}{c}8.0(\mu \mathrm{m}) \\
(\mathrm{mJy})\end{array}$ & $\begin{array}{c}24.0(\mu \mathrm{m}) \\
(\mathrm{mJy})\end{array}$ & $\begin{array}{c}70.0(\mu \mathrm{m}) \\
(\mathrm{mJy})\end{array}$ & Flags $^{\mathrm{b}}$ & Ref. \\
\hline 71 & $2235008+7515364$ & & $22.0 \pm 1.1$ & $23.4 \pm 1.1$ & $25.7 \pm 1.2$ & $32.3 \pm 1.5$ & 104. \pm 10 & 146. \pm 14 & FIM & \\
\hline 72 & $2235025+7517584$ & [RD95] 3 & $35.6 \pm 1.8$ & $43.1 \pm 2.1$ & $45.6 \pm 2.2$ & $47.6 \pm 2.3$ & $67.1 \pm 6.3$ & & FIM & 14 \\
\hline 73 & $2235042+7518206$ & & $32.4 \pm 1.6$ & $32.0 \pm 1.5$ & $36.0 \pm 1.7$ & $40.8 \pm 2.0$ & $83.7 \pm 7.8$ & 106. \pm 11 & FIM & \\
\hline 74 & $2235096+7516094$ & & $7.96 \pm 0.40$ & $8.40 \pm 0.41$ & $6.19 \pm 0.32$ & $5.13 \pm 0.24$ & $12.9 \pm 1.2$ & & FIM & \\
\hline 75 & $2235141+7515026$ & & $1.13 \pm 0.06$ & $1.50 \pm 0.08$ & $1.69 \pm 0.11$ & $2.56 \pm 0.12$ & $20.4 \pm 1.9$ & 146. \pm 14 & FI & \\
\hline 76 & $2235167+7518470$ & [K93b] 39 & $75.9 \pm 4.0$ & $74.2 \pm 3.8$ & $73.0 \pm 3.6$ & $85.9 \pm 4.2$ & 109. \pm 10 & $58.5 \pm 6.8$ & FIM & $9 ; 15$ \\
\hline 77 & $2235254+7517561$ & & 102. \pm 5 & 108. \pm 5 & 121. \pm 6 & 123. \pm 6 & $78.2 \pm 7.7$ & $\ldots$ & FIM & \\
\hline 78 & $2235266+7516369$ & & $34.5 \pm 1.8$ & $53.2 \pm 2.6$ & $58.0 \pm 2.8$ & $54.1 \pm 2.7$ & $79.2 \pm 8.1$ & $\ldots$ & FIM & \\
\hline 79 & $2235272+7518018$ & & $37.2 \pm 1.9$ & $34.2 \pm 1.7$ & $33.0 \pm 1.6$ & $35.5 \pm 1.7$ & $48.8 \pm 5.1$ & & FIM & \\
\hline 80 & $2236056+7518325$ & [K93b] 2; [K93c] 9; [K98b] 105 & $78.2 \pm 4.1$ & 102. \pm 5 & 145. \pm 7 & 246. \pm 12 & 544. \pm 51 & 377. \pm 37 . & FIM & $8 ; 9 ; 15$ \\
\hline 81 & $2236355+7521351$ & [K93c] 10; [K98b] 107 & $54.8 \pm 2.7$ & $54.9 \pm 2.8$ & $58.1 \pm 2.8$ & $72.6 \pm 3.7$ & $210 . \pm 20$ & $\ldots$ & FIM & $8 ; 9 ; 15$ \\
\hline 82 & $2236591+7521207$ & & $2.87 \pm 0.19$ & $2.38 \pm 0.14$ & $2.11 \pm 0.13$ & $2.06 \pm 0.11$ & $2.47 \pm 0.32$ & $\ldots$ & FIm & \\
\hline 83 & $2237496+7504063$ & [K93b] 2 & $18.9 \pm 1.0$ & $13.3 \pm 0.7$ & $9.61 \pm 0.48$ & $8.06 \pm 0.39$ & $94.8 \pm 8.8$ & 212. \pm 20 & FIM & $5 ; 9 ; 15$ \\
\hline 84 & $2238116+7512144$ & & $8.52 \pm 0.42$ & $7.31 \pm 0.37$ & $8.07 \pm 0.39$ & $9.46 \pm 0.46$ & $28.3 \pm 2.6$ & $50.2 \pm 5.4$ & FIM & 5 \\
\hline 85 & $2238152+7507203$ & & $13.9 \pm 0.7$ & $11.8 \pm 0.6$ & $10.1 \pm 0.5$ & $9.48 \pm 0.45$ & $16.9 \pm 1.6$ & $20.9 \pm 3.4$ & FIM & 5 \\
\hline 86 & $2238188+7511536$ & & 109. \pm 9 & 154. \pm 9 & 227. \pm 11 & $285 . \pm 27$ & $321 . \pm 30$. & 204. \pm 19 & FIM & $5 ; 15$ \\
\hline 87 & $2238296+7514265$ & & $8.62 \pm 0.43$ & $6.98 \pm 0.35$ & $6.73 \pm 0.34$ & $8.28 \pm 0.42$ & $10.7 \pm 1.0$ & .. & FIM & 5 \\
\hline 88 & $2238405+7508412$ & & $7.38 \pm 0.44$ & $6.76 \pm 0.35$ & $6.24 \pm 0.31$ & $6.42 \pm 0.31$ & $9.46 \pm 0.88$ & $29.1 \pm 3.8$ & FIM & 5 \\
\hline 89 & $2238428+7511367$ & L1251B IRS 4 & $3.91 \pm 0.40$ & $7.96 \pm 0.47$ & $8.94 \pm 0.65$ & $13.2 \pm 0.7$ & 391. \pm 37 & $8690 . \pm 803$ & FIm & 5 \\
\hline 90 & $2238469+7511337$ & L1251B IRS 1; [K93c] 14; [K98b] 111 & $30.9 \pm 2.0$ & 115. \pm 6 & $269 . \pm 13$ & 376. \pm 20 & $2790 . \pm 274$ & 19400. \pm 1790 & FIm & $5 ; 8 ; 9$ \\
\hline 91 & $2238507+7510351$ & L1251B IRS 8 & $4.10 \pm 0.21$ & $3.50 \pm 0.17$ & $3.05 \pm 0.16$ & $2.74 \pm 0.13$ & $3.66 \pm 0.38$ & .. & FIM & 5 \\
\hline 92 & $2238530+7511235$ & L1251B IRS 2 & $6.43 \pm 0.46$ & $10.2 \pm 0.6$ & $12.0 \pm 0.7$ & $16.0 \pm 0.8$ & 463. \pm 43 & 7130. \pm 658 & FIM & 5 \\
\hline 93 & $2239047+7511010$ & L1251B IRS 9 & $1.72 \pm 0.09$ & $1.63 \pm 0.08$ & $1.63 \pm 0.09$ & $1.89 \pm 0.09$ & $3.48 \pm 0.36$ & $\ldots$ & FI & 5 \\
\hline 94 & $2239133+7512155$ & L1251B IRS 16 & $41.1 \pm 2.0$ & 112. \pm 6 & $206 . \pm 11$ & 234. \pm 11 & 343. \pm 32 & 592. \pm 55 & FI & 5 \\
\hline 95 & $2239147+7507161$ & & $6.84 \pm 0.35$ & $4.65 \pm 0.23$ & $3.22 \pm 0.17$ & $1.99 \pm 0.10$ & $0.870 \pm 0.155$ & $\ldots$ & $\mathrm{Fm}$ & \\
\hline 96 & $2239273+7510282$ & [K93b] 45; [K93c] 16; [TW96] H1a & $38.3 \pm 1.9$ & $32.1 \pm 1.6$ & $29.8 \pm 1.4$ & $33.1 \pm 1.6$ & $67.2 \pm 6.2$ & 123. \pm 12 & FIM & $5 ; 9 ; 12 ; 15$ \\
\hline 97 & $2239403+7513215$ & [K93b] 46 & $110 . \pm 9$ & 162. \pm 13 & $245 . \pm 12$ & 267. \pm 13 & $280 . \pm 26$ & 197. \pm 18 & FIM & $5 ; 9 ; 15$ \\
\hline 98 & $2239464+7512585$ & & $18.4 \pm 0.9$ & $18.3 \pm 0.9$ & $19.1 \pm 0.9$ & $18.5 \pm 0.9$ & $11.2 \pm 1.0$ & $\ldots$ & FIM & $5 ; 15$ \\
\hline 99 & $2241047+7510496$ & & $4.20 \pm 0.21$ & $2.79 \pm 0.14$ & $1.98 \pm 0.11$ & $1.31 \pm 0.07$ & $3.88 \pm 0.45$ & $\ldots$ & FM & \\
\hline \multicolumn{11}{|c|}{ 2. IRAC YSO Candidates } \\
\hline 100 & $2100224+6813042$ & & $2.22 \pm 0.14$ & $4.43 \pm 0.24$ & $5.10 \pm 0.25$ & $5.91 \pm 0.29$ & $<23.0$ & $\ldots$ & $\mathrm{fI}$ & \\
\hline 101 & $2101030+6813072$ & & $10.9 \pm 0.5$ & $11.9 \pm 0.6$ & $9.88 \pm 0.47$ & $12.3 \pm 0.6$ & $<16.2$ & $\ldots$ & fI & \\
\hline 102 & $2101102+6811332$ & & $1.63 \pm 0.08$ & $1.39 \pm 0.07$ & $1.28 \pm 0.07$ & $1.39 \pm 0.09$ & $<3.39$ & $\ldots$ & I & \\
\hline 103 & $2101125+6810195$ & NGC 7023 S I & $34.2 \pm 1.7$ & $27.4 \pm 1.4$ & $26.4 \pm 1.3$ & $33.6 \pm 1.8$ & $<55.2$ & $\ldots$ & fI & 7 \\
\hline 104 & $2101361+6808226$ & NGC 7023 S E & $24.1 \pm 1.2$ & $31.1 \pm 1.5$ & $29.2 \pm 1.6$ & $55.2 \pm 4.4$ & $<89.3$ & $\ldots$ & fI & $7 ; 15$ \\
\hline 105 & $2228018+6901191$ & L1221 IRS 2 & $83.6 \pm 4.5$ & 137. \pm 9 & $324 . \pm 16$ & $381 . \pm 23$ & $<368$ & $\ldots$ & fI & 4 \\
\hline
\end{tabular}


Table 6

(Continued)

\begin{tabular}{|c|c|c|c|c|c|c|c|c|c|c|}
\hline Id. & $\begin{array}{c}\text { Name/Position } \\
\text { (SSTgbsJ) }\end{array}$ & Literature Name $^{\mathrm{a}}$ & $\begin{array}{c}3.6(\mu \mathrm{m}) \\
(\mathrm{mJy})\end{array}$ & $\begin{array}{c}4.5(\mu \mathrm{m}) \\
(\mathrm{mJy})\end{array}$ & $\begin{array}{c}5.8(\mu \mathrm{m}) \\
(\mathrm{mJy})\end{array}$ & $\begin{array}{c}8.0(\mu \mathrm{m}) \\
(\mathrm{mJy})\end{array}$ & $\begin{array}{c}24.0(\mu \mathrm{m}) \\
(\mathrm{mJy})\end{array}$ & $\begin{array}{c}70.0(\mu \mathrm{m}) \\
(\mathrm{mJy})\end{array}$ & Flags $^{\mathrm{b}}$ & Ref. \\
\hline 106 & $2235323+7518377$ & & $1.84 \pm 0.10$ & $1.71 \pm 0.09$ & $1.51 \pm 0.10$ & $1.66 \pm 0.08$ & $<2.07$ & $\ldots$ & fI & \\
\hline 107 & $2238424+7511454$ & L1251B IRS 3; [K93b] 44; [TW96 H2b] & $15.5 \pm 0.9$ & $12.5 \pm 0.6$ & $11.0 \pm 0.7$ & $10.5 \pm 0.6$ & $<80.2$ & $\ldots$ & fI & $5 ; 12 ; 15$ \\
\hline 108 & $2238440+7511266$ & L1251B IRS 5 & $9.89 \pm 0.50$ & $8.02 \pm 0.39$ & $5.50 \pm 0.30$ & $5.12 \pm 0.28$ & $<27.2$ & $\ldots$ & fI & 5 \\
\hline 109 & $2238481+7511486$ & L1251B IRS 6 & $5.64 \pm 0.29$ & $5.99 \pm 0.29$ & $6.55 \pm 0.35$ & $6.84 \pm 0.35$ & $<10.8$ & $\ldots$ & fI & 5 \\
\hline \multicolumn{11}{|c|}{ 3. 2MASS-MIPS YSO Candidates } \\
\hline 110 & $2031100+6700273$ & & $\ldots$ & $\ldots$ & $\ldots$ & $\ldots$ & $1.15 \pm 0.21$ & $\ldots$ & $\mathrm{m}$ & \\
\hline 111 & $2032327+6712207$ & & $\ldots$ & $\ldots$ & $\ldots$ & $\ldots$ & $24.0 \pm 2.3$ & $\ldots$ & $\mathrm{m}$ & \\
\hline 112 & $2034190+6714289$ & & $\ldots$ & $\ldots$ & $\ldots$ & $\ldots$ & $0.905 \pm 0.164$ & $\ldots$ & $\mathrm{m}$ & \\
\hline 113 & $2035480+6802564$ & & $\ldots$ & $\ldots$ & $\ldots$ & $\ldots$ & $3.22 \pm 0.36$ & $\ldots$ & $\mathrm{m}$ & \\
\hline 114 & $2037403+6656231$ & & $\ldots$ & $\ldots$ & $\ldots$ & $\ldots$ & $4.00 \pm 0.41$ & $62.6 \pm 8.4$ & M & \\
\hline 115 & $2038524+6702468$ & & $1.02 \pm 0.06$ & $\ldots$ & $0.481 \pm 0.045$ & $\ldots$ & $1.04 \pm 0.17$ & $\ldots$ & $\mathrm{m}$ & \\
\hline 116 & $2039047+6731116$ & & $\ldots$ & $2.56 \pm 0.12$ & $\ldots$ & $1.39 \pm 0.07$ & $1.50 \pm 0.22$ & $\ldots$ & $\mathrm{m}$ & \\
\hline 117 & $2044098+6720237$ & \multirow[t]{6}{*}{$\mathrm{V}^{*}$ FI Cep } & $\ldots$ & $\ldots$ & $\ldots$ & $\ldots$ & $59.7 \pm 5.5$ & $\ldots$ & $\mathrm{m}$ & \\
\hline 118 & $2047218+6748507$ & & $\ldots$ & $\ldots$ & $\ldots$ & $\ldots$ & $3.44 \pm 0.44$ & $\ldots$ & $\mathrm{m}$ & \\
\hline 119 & $2048103+6803019$ & & $\ldots$ & $\ldots$ & $\ldots$ & $\ldots$ & $3.27 \pm 0.38$ & $\ldots$ & $\mathrm{m}$ & \\
\hline 120 & $2053476+6818039$ & & $\ldots$ & $\ldots$ & $\ldots$ & $\ldots$ & $26.4 \pm 2.5$ & $60.3 \pm 7.2$ & M & \\
\hline 121 & $2054530+6820374$ & & $\ldots$ & $\ldots$ & $\ldots$ & $\ldots$ & $26.2 \pm 2.4$ & $\ldots$ & M & \\
\hline 122 & $2056331+6814482$ & & $\ldots$ & $\ldots$ & $\ldots$ & $\ldots$ & $3.25 \pm 0.35$ & $54.5 \pm 6.9$ & $\mathrm{~m}$ & \\
\hline 123 & $2058467+7740256$ & [K98c] EM* 30 & $\ldots$ & $\ldots$ & $\ldots$ & $\ldots$ & 115. \pm 11 & $\ldots$ & M & $8 ; 15$ \\
\hline 124 & $2100529+7703149$ & L1228 S5; [K98b] 24; [K98c] EM* 35 & $\ldots$ & $55.4 \pm 2.9$ & $\ldots$ & $38.4 \pm 2.5$ & $95.9 \pm 8.9$ & $64.8 \pm 10.4$ & M & $3 ; 8 ; 15$ \\
\hline 125 & $2102204+6825241$ & V* FV Cep; $[$ K98c] Em* 38 & $\ldots$ & $83.4 \pm 4.1$ & $\ldots$ & 116. \pm 6 & 104. \pm 10 & $\ldots$ & M & $8 ; 15$ \\
\hline 126 & $2103416+6823457$ & & $\ldots$ & $3.84 \pm 0.18$ & $\ldots$ & $2.93 \pm 0.14$ & $3.88 \pm 0.40$ & $\ldots$ & M & \\
\hline 127 & $2104282+7703171$ & & $\ldots$ & $\ldots$ & $\ldots$ & $\ldots$ & $34.4 \pm 3.2$ & $51.7 \pm 6.4$ & M & \\
\hline 128 & $2105593+7642272$ & & $1.32 \pm 0.06$ & $\ldots$ & $0.787 \pm 0.049$ & $\ldots$ & $1.72 \pm 0.19$ & $\ldots$ & $\mathrm{m}$ & \\
\hline 129 & $2156133+7658142$ & & $\ldots$ & $\ldots$ & $\ldots$ & $\ldots$ & $4.68 \pm 0.47$ & $33.8 \pm 7.3$ & $\mathrm{~m}$ & \\
\hline 130 & $2210100+7525255$ & & $\ldots$ & $\ldots$ & $\ldots$ & $\ldots$ & $0.805 \pm 0.266$ & $\ldots$ & $\mathrm{m}$ & \\
\hline 131 & $2214393+7520584$ & & $\ldots$ & $\ldots$ & $\ldots$ & $\ldots$ & $0.881 \pm 0.154$ & $\ldots$ & $\mathrm{m}$ & \\
\hline 132 & $2217097+7645029$ & & $\ldots$ & $\ldots$ & $\ldots$ & $\ldots$ & $1.63 \pm 0.30$ & $\ldots$ & $\mathrm{m}$ & \\
\hline 133 & $2236029+7523260$ & & $\ldots$ & $\ldots$ & $\ldots$ & $\ldots$ & $7.40 \pm 0.70$ & $\ldots$ & M & \\
\hline \multicolumn{11}{|c|}{ 4. Known YSO Candidates } \\
\hline 134 & $2039062+6802150$ & L1157 MM; [K98b] 7; HH 365 & $1.34 \pm 0.29$ & $4.87 \pm 0.49$ & $3.60 \pm 0.51$ & $2.29 \pm 0.44$ & $80.3 \pm 7.5$ & 10700. \pm 1160 & $\ldots$ & 8 \\
\hline 135 & $2045539+6757387$ & V* PV Cep; [K98b] 10; [K98c] EM* 9; HH 215 & $\ldots$ & $\ldots$ & $\ldots$ & $\ldots$ & $\ldots$ & $\ldots$ & $\ldots$ & 8 \\
\hline 136 & $2101251+7706541$ & L1228 S1(b) & $3.67 \pm 0.18$ & $2.95 \pm 0.14$ & $2.27 \pm 0.12$ & $1.37 \pm 0.07$ & $<0.179$ & $\ldots$ & $\ldots$ & $3 ; 15$ \\
\hline 137 & $2101271+6810380$ & NGC7023 RS 2 & $47.7 \pm 2.5$ & $43.2 \pm 2.1$ & $59.6 \pm 3.2$ & $<56.6$ & $<20.7$ & $\ldots$ & $\ldots$ & 15 \\
\hline 138 & $2101369+6809479$ & HD 200775; V* V380 Cep; NGC 7023 S A & $\ldots$ & $2360 . \pm 271$ & 10700. \pm 887 & $7160 . \pm 406$ & $\ldots$ & $\ldots$ & $\ldots$ & 7 \\
\hline 139 & $2101436+6809361$ & NGC7023 RS S3; NGC 7023 S D & $28.4 \pm 1.4$ & $18.8 \pm 0.9$ & $13.1 \pm 0.7$ & $6.65 \pm 0.46$ & $\ldots$ & $\ldots$ & $\ldots$ & 15 \\
\hline 140 & $2101493+7657481$ & IRAS F21025+7645; [K98b] 32 & $<0.00552$ & $<0.00546$ & $\ldots$ & $<0.00645$ & $<0.0264$ & $\ldots$ & $\ldots$ & 8 \\
\hline
\end{tabular}


Table 6

(Continued)

\begin{tabular}{|c|c|c|c|c|c|c|c|c|c|c|}
\hline Id. & $\begin{array}{c}\text { Name/Position } \\
\text { (SSTgbsJ) }\end{array}$ & Literature Name $^{\mathrm{a}}$ & $\begin{array}{c}3.6(\mu \mathrm{m}) \\
(\mathrm{mJy})\end{array}$ & $\begin{array}{c}4.5(\mu \mathrm{m}) \\
(\mathrm{mJy})\end{array}$ & $\begin{array}{c}5.8(\mu \mathrm{m}) \\
(\mathrm{mJy})\end{array}$ & $\begin{array}{c}8.0(\mu \mathrm{m}) \\
(\mathrm{mJy})\end{array}$ & $\begin{array}{c}24.0(\mu \mathrm{m}) \\
(\mathrm{mJy})\end{array}$ & $\begin{array}{c}70.0(\mu \mathrm{m}) \\
(\mathrm{mJy})\end{array}$ & Flags $^{\mathrm{b}}$ & Ref. \\
\hline 141 & $2103121+6813012$ & IRAS F21025+6801; [K98b] 31 & & & & & $<0.140$ & & $\cdots$ & 8 \\
\hline 142 & $2234119+7518099$ & [K93b] 1; GSC 04600-02450 & 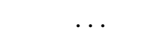 & $91.3 \pm 14.6$ & 686. \pm 35 & 474. \pm 31 & $1440 . \pm 137$ & $1740 . \pm 162$ & $\ldots$ & $9 ; 15$ \\
\hline 143 & $2235234+7517077$ & [TW96] N1a; [K98b] 103; [K93c] 8 & 373. \pm 26 & $1000 . \pm 114$ & 1860. \pm 119 & $\ldots$ & $\ldots$ & $\ldots$ & $\ldots$ & $8 ; 9 ; 12$ \\
\hline
\end{tabular}

Notes.

${ }^{a}$ For c2d papers (refs 1-5) the literature name, where one is given, is taken as the core name followed by the index or name given to the source in the actual paper.

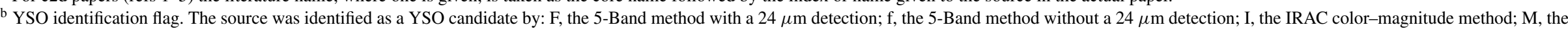
2MASS/MIPS color-magnitude method (greater than 1 mag away from the limits) and m, the 2MASS/MIPS color-magnitude method (less than 1 mag away from the limits).

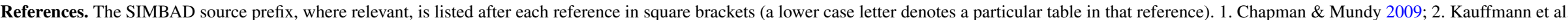

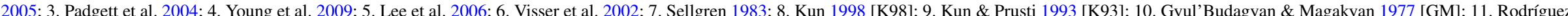
\& Reipurth 1996 [RR96]; 12. Tóth \& Walmsley 1996 [TW96]; 13. Rodríguez \& Reipurth 1998 [RR98]; 14. Rosvick \& Davidge 1995 [RD95]; 15. Kun et al. 2008.

(This table is available in its entirety in a machine-readable form in the online journal. A portion is shown here for guidance regarding its form and content.) 

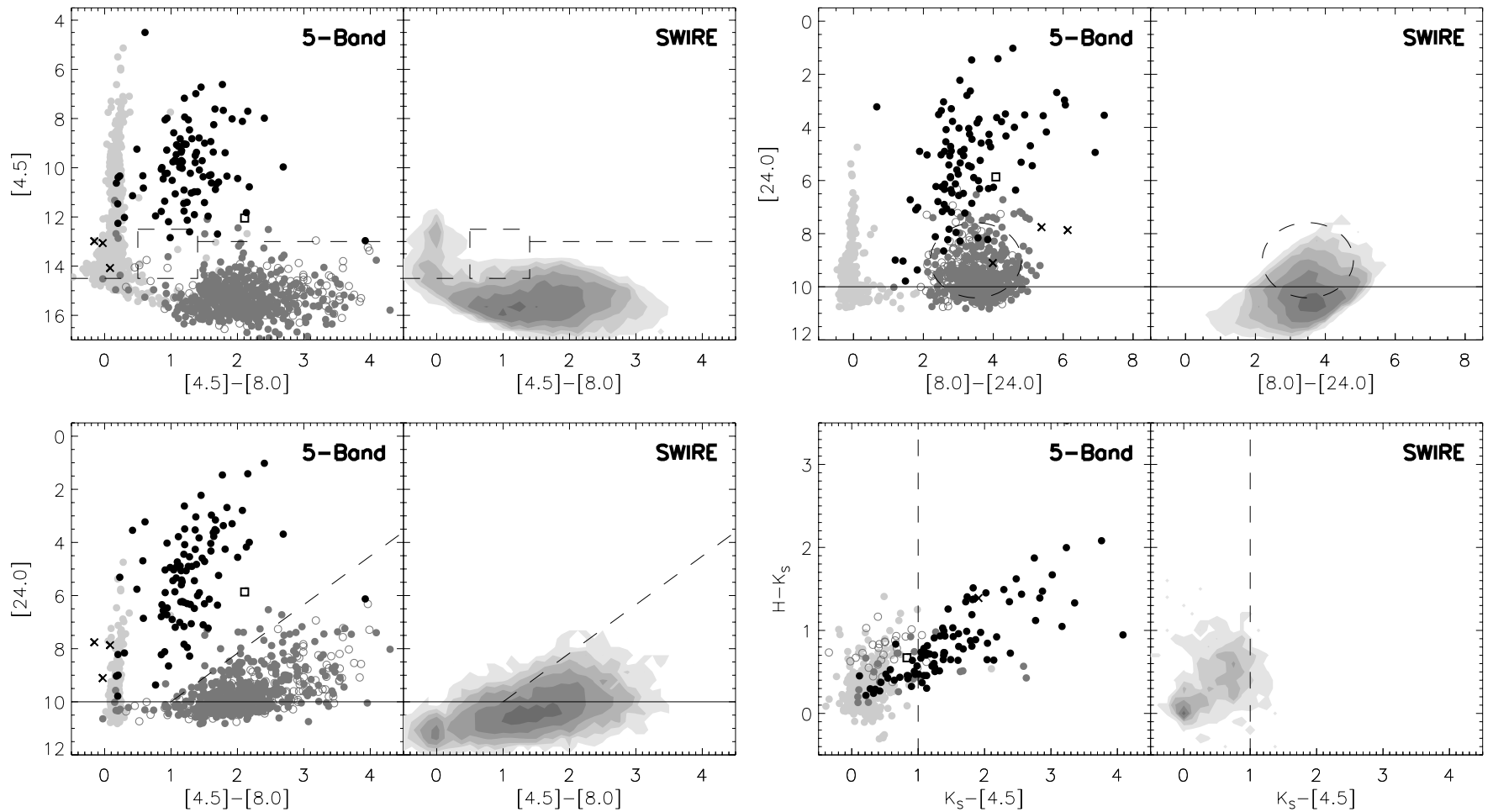

Figure 6. Color-magnitude diagrams after Harvey et al. (2007) showing the separation of YSO and galaxy candidates and the dividing lines that were used to construct $P_{\text {gal }}$. Left panels: SGBS Cepheus data. The markers show point-line/extended YSOs (black filled circles/crosses) and point-like/extended background galaxy candidates (dark gray filled/open circles) that have been separated via their $P_{\text {gal }}$ value. Stellar sources (light gray filled circles) with the same $\mathrm{S} / \mathrm{N}$ requirements as the YSOs and galaxies are shown for comparison. The open square is a source with only an $8 \mu \mathrm{m}$ flux upper limit, but otherwise satisfies the 5-Band YSO selection criterion. Right panels: contour plots of the number density of sources identified as either YSOs or stars from the SWIRE catalog. Dashed black lines show the "fuzzy" borders used to calculate $P_{\text {gal }}$, while solid lines show the hard [24.0] $<10$ mag cutoff.

[4.5]-[8.0] versus [8.0] for sources without an MIPS $24 \mu \mathrm{m}$ detection and comparative plots of the 5-Band and SWIRE sources. Of the YSOs identified by the 5-Band scheme, 12 would be misidentified by the IRAC-only scheme as stars due to their small [4.5]-[8.0] color and six bright galaxies would have crept into the sample.

The sources that satisfy the IRAC-only scheme are listed with an I flag in Column 10 of Table 6 . A source flagged with a lowercase $\mathrm{f}$ has a $24 \mu \mathrm{m}$ upper limit flux, but would otherwise satisfy the 5-Band scheme. A comparison of sources with I and $f$ flags shows that the IRAC-only scheme and the 5-Band scheme are consistent for sources without a $24 \mu \mathrm{m}$ detection. A total of 10 additional YSO candidates were identified by the IRAC-only scheme.

An additional constraint can be imposed in color-space as external galaxies are often rich in PAH emission compared with YSO sources. Gutermuth et al. (2008) proposed using the [4.5]-[8.0] versus [3.6]-[5.8] and [5.8]-[8.0] versus [4.5]-[5.8] color-color diagrams to reject background galaxies with strong PAH emission. The sources that match these rules are shown with triangle rather than circle markers in Figure 7. No IRAC YSO candidates have evidence of PAH emission from this technique, but two 5-Band YSO candidates are flagged. One of these is L1148-IRS.

\subsubsection{MASS/MIPS Identifications}

An inherent strength of the 5-Band scheme is that it relies on a broad range of color combinations, but it can only be used in regions where there is IRAC coverage. Rebull et al. (2007) proposed a scheme to identify YSOs using MIPS $24 \mu \mathrm{m}$ and 2MASS $K_{s}$ and the selection rules $K_{s}-[24]>2$ and
$K_{s}<14$. To this we add the limit [24] $<10$ for consistency with the 5-Band scheme. The lower row of Figure 7 shows colormagnitude plots of $K_{s}-$ [24] versus $K_{s}$ for sources without an IRAC detection and comparative plots of the 5-Band and SWIRE sources. Column 10 of Table 6 includes an M flag for the sources that would have been identified as a YSO candidate by this method. A significant fraction of 2MASS/MIPS YSO candidates were found within 1 magnitude of the selection limits. These are marked as open circles in Figure 7 and listed with a lowercase $\mathrm{m}$ in column 10 .

The 2MASS/MIPS scheme would have misidentified 19 of the 5-Band YSO candidates as background galaxies and stars. From Figure 7, we can see that the majority of these appear in the region of background galaxies with a $K_{s}-$ [24] color of $\sim 10$. This is approximately equivalent to the spectral index of an embedded protostellar source (a Class I YSO, see the next section and Figure 8). This shows a further advantage of the 5-Band scheme in that it is more sensitive to embedded sources as it does not rely heavily on $K_{s}$. Based on the MIPS scheme, 4-5 galaxies could have crept into the YSO sample. A total of 24 additional YSO candidates were identified by the 2MASS/MIPS scheme that were not identified by the 5-Band and IRAC-Only schemes.

\subsubsection{The Final Catalog}

The morphologies of all YSO candidates were checked at each wavelength listed in Table 3 to screen for obvious galaxy candidates or possible artifacts. During the visual checking, an unusual extended source was found at $21^{\mathrm{h}} 02^{\mathrm{m}} 21^{\mathrm{s}} .2+68^{\circ} 04^{\prime} 36^{\prime \prime}$. It is a flat spectrum source across the 2-24 $\mu \mathrm{m}$ waveband, but rises at longer wavelengths. It is detected in all three MIPS 

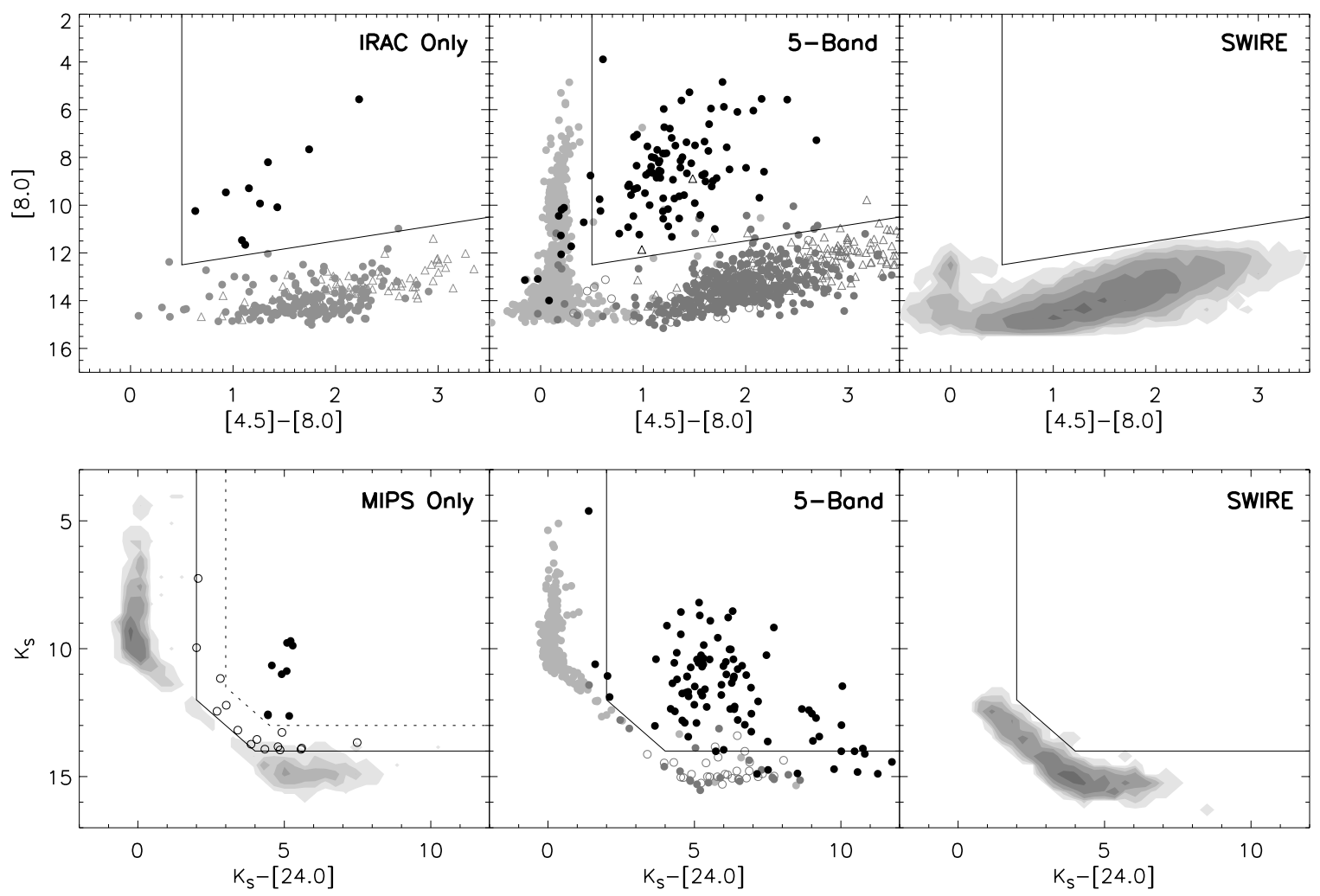

Figure 7. Color-magnitude diagrams for IRAC and 2MASS/MIPS detections showing the dividing lines used to separate YSO candidates from other sources. Top row: IRAC-only detections. The left panel shows the IRAC-only data; the middle and right panels the 5-Band and SWIRE galaxies as per Figure 6. The triangle markers show PAH-strong sources as identified by the selection rules from Gutermuth et al. (2008). Lower row: 2MASS/MIPS only detections. Left panel shows 2MASS/MIPS-only detections; middle and right panels are 5-Band detections and SWIRE galaxies as per Figure 6. The solid lines show the selection cutoffs. Dashed lines show a 1 mag offset from the selection cutoffs and open markers show YSO candidates within that zone. Non-YSOs in some panels have been shown as a density plot for clarity.

wavebands. It was coincident with a spur in the extended $160 \mu \mathrm{m}$ emission from the NGC 7023 nebula and a small increase in visual extinction. It corresponds with a $1.4 \mathrm{GHz}(20 \mathrm{~cm})$ source NVSS 210221+680436 (Condon et al. 1998). Its colors of $[5.8]-[8.0]=2.8$ and [3.6]-[4.5] $=0.0$ place it well away from the color-color clustering of the other YSO candidates (see Section 4.3). This object has been excluded from further consideration as a YSO as it is probably a background galaxy.

A total of 133 YSO candidates were identified by the three schemes. The vast majority of these (99) were identified by the 5-Band scheme, and a further 10 were identified by the IRAC scheme. The 2MASS/MIPS data identified a further 24 candidates. Although the 2MASS/MIPS data covered a larger area than the 5-Band scheme, most of it was at a lower visual extinction. Thus, it could be argued that we would not expect to detect the same density of YSOs. The position of each YSO candidate was checked against the SIMBAD database. The SIMBAD name and other selected literature names for each source are listed in Column 3 of Table 6. A total of 59 YSO candidates were found to have antecedent catalog names.

It should be noted that not all known YSOs and YSO candidates coincident with our mapped area were identified by the three YSO candidate identification schemes. Section 6.2 discusses the completeness of our YSO sample, and Table 16 lists 15 known sources missed by our selection criteria. These include luminous YSOs such as HD 200775 (the driving source of the NGC 7023 nebula) and PV Cep (a "runaway" YSO; Goodman \& Arce 2004), which were excluded because they saturate the Spitzer detectors. In addition, sources like XMMU J223727.7+751725 were excluded since they have no detectable infrared (2MASS/Spitzer) flux. From this list, 10 are coincident with entries in the Cepheus catalog, but which were not identified by the three Spitzer schemes. Photometry for these sources is listed in the fourth section of Table 6. We refer to these 10 sources as non-Spitzer-identified YSO candidates.

Hereafter, we assume that these 143 (133 Spitzer plus 10 previously identified) YSO candidates are actually YSOs and analyze their properties accordingly.

\subsection{YSO Classification}

YSOs can be separated into a series of four evolutionary classes depending on either their infrared spectral index (Wilking et al. 1989) or the mean frequency of their SED (Myers \& Ladd 1993). The spectral index classification of a source as a Class II or Class III YSO is generally correlated with its respective classification as a Classical T Tauri (CTTS) or Weak-Line T Tauri (WTTS) star (Wilking et al. 1989; André \& Montmerle 1994). Class 0 and Class I protostars are younger embedded YSOs and are differentiated from each other by the amount of material remaining in their envelopes. The envelope appears as a colder component to the SED and peaks toward the submillimeter. The original definition of a Class 0 protostar was an object whose submillimeter luminosity $(\lambda>350 \mu \mathrm{m})$ contributed greater than $0.5 \%$ to the source's total bolometric luminosity, which is equivalent to a protostar-to-envelope mass ratio of less than one (André et al. 1993). The second method of YSO classification uses a bolometric temperature calculated for 


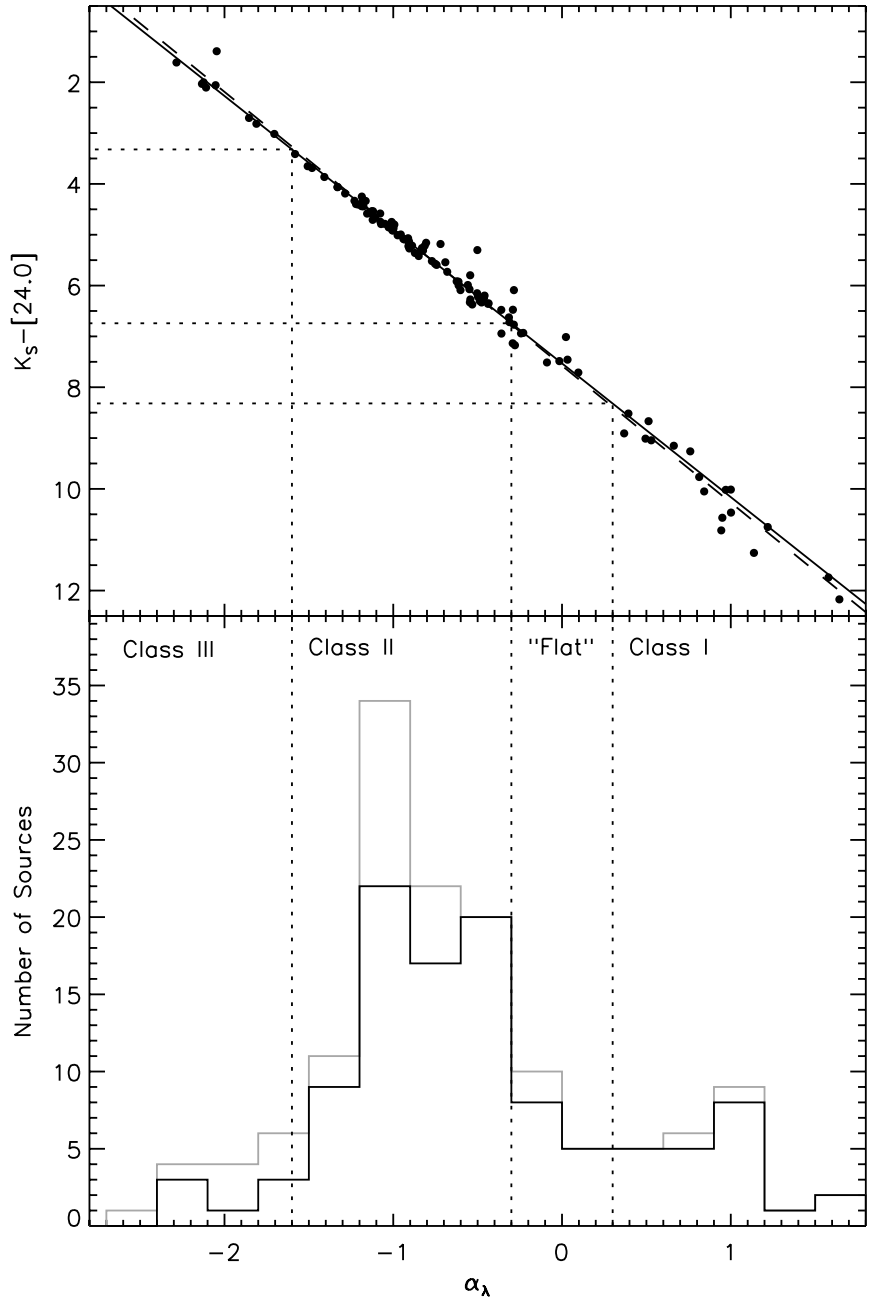

Figure 8. Upper: plot showing the equivalence of $\alpha_{\lambda}$ and the $K_{s}$-[24.0] color for the 5-Band YSOs. The solid line shows the theoretical relationship between $\alpha_{\lambda}$ and $K_{s}$-[24.0]. The dashed line is a linear regression to the data. Lower: histogram of spectral indices for YSO candidates. The black line shows those YSOs detected by IRAC. The gray line shows all YSOs including the 2MASS/ MIPS YSOs. The dotted lines show the divisions of the classical YSO spectral classes and are extended to show the equivalent $K_{s}$-[24.0] colors in the top diagram. The majority of YSOs have indices in the Class II regime. Not all of the YSOs included in the solid histogram are plotted in the upper graph as not all IRAC YSOs have $24 \mu \mathrm{m}$ detections.

a blackbody that has the same mean frequency as the source's SED. The value of the bolometric temperature decreases from Class III to 0 as the SED is increasingly dominated by long wavelength emission. The results for this method are described in Section 5.2.

Table 7 lists the derived properties of the YSOs from Table 6. The first column lists the index number of the YSO. Column 2 lists the dark cloud from Figure 1 with which the YSO candidate is associated. Locations that have a visual extinction less than one magnitude in the Dobashi et al. (2005) maps or are not part of an identifiable YSO group (see Section 6.3) are listed as "offcloud." Column 3 lists the spectral index of the source, $\alpha_{\mathrm{IR}}$, as given by

$$
\alpha_{\mathrm{IR}}=\frac{d \log \left(\lambda F_{\lambda}\right)}{d \log \lambda}
$$

where $F_{\lambda}$ is the monochromatic flux density at wavelength $\lambda$. The index was calculated by a least-squares fit to all available data in the range 2MASS $K_{s}(2.2 \mu \mathrm{m})$ to MIPS $24 \mu \mathrm{m}$.
The mapping between the YSO evolutionary sequence and the spectral index proposed by Wilking et al. (1989) and amended by Greene et al. (1994) and André \& Montmerle (1994) is Class I $\left(\alpha_{\lambda} \leqslant 0.3\right)$, "flat" $\left(0.3>\alpha_{\lambda} \geqslant-0.3\right)$, Class II $\left(-0.3>\alpha_{\lambda} \geqslant-1.6\right)$, and Class III $\left(-1.6>\alpha_{\lambda}\right)$. The decreasing spectral index is attributed to the decline in the amount of circumstellar dust with advancing YSO evolution. The associated class for each source is listed in Column 4 of Table 7. "Flat" spectrum sources have spectra intermediate between embedded and T Tauri stages. Class 0 YSOs cannot be distinguished from Class I YSOs via this method as it is not sensitive to the long wavelength part of the SED where the majority of the envelope emission radiates (Enoch et al. 2009). Radiative transfer modeling of YSO SEDs has shown that the amount of dust observed around a YSO, and thus its spectral index, will depend on the angle of inclination of the circumstellar disk to the observer (Robitaille et al. 2006; Crapsi et al. 2008). This degeneracy could possibly be broken by using longer wavelength data (see Section 5.2 for a comparison between the number of YSOs classified by each method).

The majority of the data points used to calculate $\alpha_{\mathrm{IR}}$ are in the IRAC regime. For those YSO candidates without an IRAC detection, $\alpha_{\mathrm{IR}}$ is only calculated from 2MASS $K_{s}$ and MIPS $24 \mu \mathrm{m}$-effectively the $K_{s}$-[24] color. To test the robustness of using just the 2MASS/MIPS color, the top of Figure 8 shows $K_{s}$-[24] versus $\alpha_{\text {IR }}$ for the YSO candidates detected with IRAC. The tight correlation follows a linear relationship, thus proving that the equivalent color can be an acceptable proxy for a spectral index calculated using more intermediate points.

The solid line through the points in Figure 8 shows the theoretical relationship between $K_{s}{ }^{-}[24.0]$ and $\alpha_{\lambda}$. For two generic bands, $\mathrm{A}$ and $\mathrm{B}$, the color and the spectral index are linearly related such that $m_{\mathrm{A}}-m_{\mathrm{B}}=C_{1} \alpha_{\lambda}+C_{2}$, where $C_{1}=-2.5 X, C_{2}=-2.5+2.5 Y, X=\log \left(\lambda_{\mathrm{A}}\right)-\log \left(\lambda_{\mathrm{B}}\right)$, and $Y=\log \left(F_{0, \mathrm{~A}}\right)-\log \left(F_{0, \mathrm{~B}}\right)$. The wavelength of each band is $\lambda_{\mathrm{A}}$ and $\lambda_{\mathrm{B}}$, the flux zero points are $F_{0, \mathrm{~A}}$ and $F_{0, \mathrm{~B}}$, and the color is $\left(m_{\mathrm{A}}-m_{\mathrm{B}}\right)$. Using the data from Table 3 , the coefficients for $K_{s}$-[24.0] are $C_{1}=2.63$ and $C_{2}=7.55$. A linear regression to the data, shown by the dashed line, gives coefficients of $C_{1}=2.64 \pm 0.06$ and $C_{2}=7.73 \pm 0.06$.

The color- $\alpha_{\text {IR }}$ equation can be used to recompute the class boundaries in terms of a source's $K_{s}$-[24.0] color such that they become $K_{s}$-[24.0] $=8.32,6.74$, and 3.32 for the boundaries between Class I/"flat," "flat"/Class II, and Class II/III, respectively. These values agree with those used by Lee et al. (2006) and Rebull et al. (2007). These recomputed boundaries are shown in Figure 8 by the dotted lines.

The lower panel of Figure 8 shows a histogram of the YSO spectral indices. The black line histogram shows sources with IRAC coverage. The range of values of $\alpha_{\lambda}$ is -2.28 to 1.63 . The gray histogram shows all sources and includes 2MASS/MIPS YSOs. Both distributions peak around -1 and show an increase in the number of sources from Class I to Class II. The peak of the Cepheus spectral index histograms matches the peak of the distributions seen in Serpens (Harvey et al. 2007), Lupus (Merín et al. 2008), and IC 5146 (Harvey et al. 2008). The range of extreme spectral indices is less than that in IC 5146 (Harvey et al. 2008).

The number of sources classified in each class via $\alpha_{\lambda}$ and that number as a percentage of the Spitzer-identified YSOs is listed in Column 2 of Table 8. The row for Class 0 sources is left blank for $\alpha_{\mathrm{IR}}$, as they can only be distinguished by their bolometeric temperature. We can compare the relative fraction 
No. 1, 2009

Table 7

YSO Properties

\begin{tabular}{|c|c|c|c|c|c|c|c|}
\hline Index & Region & $\begin{array}{l}\alpha_{\mathrm{IR}} \\
\text { (a) } \\
\end{array}$ & $\begin{array}{c}\alpha_{\mathrm{IR}} \\
\text { Class }\end{array}$ & $\begin{array}{l}T_{\text {bol }} \\
(\mathrm{K}) \\
(\mathrm{b}) \\
\end{array}$ & $\begin{array}{c}T_{\text {bol }} \\
\text { Class } \\
\text { (c) }\end{array}$ & $\begin{array}{c}L_{\text {bol }} \\
\left(L_{\odot}\right) \\
(\mathrm{d})\end{array}$ & $\begin{array}{c}L_{\mathrm{IR}} \\
\left(L_{\odot}\right) \\
(\mathrm{e}) \\
\end{array}$ \\
\hline 1 & L1152 & 1.0 & I & 33 & 0 & 1.6 & 0.060 \\
\hline 2 & L1152 & -0.31 & II & 160 & I & 0.50 & 0.34 \\
\hline 3 & L1152 & -0.50 & II & 270 & I & 2.1 & 1.6 \\
\hline 4 & L1147 & -0.089 & $\mathrm{~F}$ & 630 & $\mathrm{~F}$ & 0.017 & 0.016 \\
\hline 5 & L1148 & 0.81 & I & 81 & I & 0.44 & 0.017 \\
\hline 6 & L1228N & -1.2 & II & 1500 & II & 0.023 & 0.019 \\
\hline 7 & L1228N & 0.66 & I & 54 & 0 & 0.22 & 0.037 \\
\hline 8 & L1228N & 0.76 & I & 35 & 0 & 0.13 & 0.019 \\
\hline 9 & $\mathrm{~L} 1228 \mathrm{~N}$ & 0.096 & $\mathrm{~F}$ & 79 & I & 2.3 & 0.90 \\
\hline 10 & L1228N & -0.61 & II & 750 & F & 0.0052 & 0.0052 \\
\hline 11 & L1228N & -0.60 & II & 820 & F & 0.093 & 0.089 \\
\hline 12 & L1228N & -0.36 & II & 670 & $\mathrm{~F}$ & 0.039 & 0.038 \\
\hline 13 & L1228N & -1.2 & II & 1600 & II & 0.13 & 0.099 \\
\hline 14 & L1228N & -1.2 & II & 1200 & II & 0.055 & 0.051 \\
\hline 15 & NGC7023 & -0.49 & II & 530 & F & 2.4 & 1.8 \\
\hline 16 & NGC7023 & -0.55 & II & 380 & $\mathrm{~F}$ & 0.36 & 0.26 \\
\hline 17 & NGC7023 B & 1.6 & I & 59 & 0 & 0.72 & 0.12 \\
\hline 18 & NGC7023 B & 0.95 & I & 49 & 0 & 0.34 & 0.12 \\
\hline 19 & NGC7023 B & -0.87 & II & 840 & $\mathrm{~F}$ & 0.095 & 0.095 \\
\hline 20 & NGC7023 B & -1.3 & II & 970 & II & 0.84 & 0.72 \\
\hline 21 & NGC7023 & -2.3 & III & 2500 & II & 0.34 & 0.19 \\
\hline 22 & L1228 S & -1.2 & II & 1600 & II & 0.014 & 0.011 \\
\hline 23 & NGC7023 A & -1.1 & II & 1600 & II & 0.099 & 0.074 \\
\hline 24 & NGC7023 A & -1.0 & II & 1500 & II & 0.17 & 0.14 \\
\hline 25 & NGC7023 A & -0.83 & II & 1400 & II & 0.24 & 0.18 \\
\hline 26 & NGC7023 A & -0.91 & II & 1200 & II & 0.027 & 0.024 \\
\hline 27 & NGC7023 A & -0.54 & II & 1000 & II & 0.73 & 0.64 \\
\hline 28 & L1228 S & -0.24 & $\mathrm{~F}$ & 590 & F & 0.011 & 0.011 \\
\hline 29 & NGC7023 A & -0.62 & II & 780 & $\mathrm{~F}$ & 0.082 & 0.082 \\
\hline 30 & L1228 S & -1.1 & II & 1700 & II & 0.92 & 0.59 \\
\hline 31 & NGC7023 A & -0.61 & II & 870 & $\mathrm{~F}$ & 0.11 & 0.10 \\
\hline 32 & L1228 S & -0.50 & II & 730 & $\mathrm{~F}$ & 0.21 & 0.13 \\
\hline 33 & L1228 S & -0.77 & II & 1000 & II & 0.20 & 0.15 \\
\hline 34 & NGC7023 A & 1.0 & I & 14 & 0 & 0.74 & 0.060 \\
\hline 35 & L1228 S & -0.44 & II & 53 & 0 & 0.15 & 0.066 \\
\hline 36 & NGC7023 A & -0.91 & II & 1100 & II & 0.35 & 0.32 \\
\hline 37 & L1228 S & -0.31 & II & 340 & I & 0.018 & 0.013 \\
\hline 38 & NGC7023 A & -0.91 & II & 1400 & II & 0.32 & 0.24 \\
\hline 39 & L1172 & -1.1 & II & 1200 & II & 0.13 & 0.12 \\
\hline 40 & NGC7023 A & 0.37 & I & 310 & I & 0.27 & 0.16 \\
\hline 41 & NGC7023 & -1.2 & II & 1300 & II & 0.11 & 0.10 \\
\hline 42 & NGC7023 A & -1.2 & II & 1500 & II & 0.34 & 0.27 \\
\hline 43 & L1228 S & -0.29 & $\mathrm{~F}$ & 560 & $\mathrm{~F}$ & 0.15 & 0.083 \\
\hline 44 & NGC7023 A & -0.50 & II & 830 & $\mathrm{~F}$ & 0.069 & 0.069 \\
\hline 45 & NGC7023 A & 0.023 & F & 1000 & II & 0.044 & 0.027 \\
\hline 46 & L1228 S & -0.46 & II & 760 & $\mathrm{~F}$ & 0.32 & 0.19 \\
\hline 47 & NGC7023 & -1.0 & II & 1200 & II & 0.077 & 0.071 \\
\hline 48 & NGC7023 & 0.53 & I & 390 & $\mathrm{~F}$ & 0.067 & 0.065 \\
\hline 49 & L1172 & 1.1 & I & 42 & 0 & 0.58 & 0.13 \\
\hline 50 & L1172 & -0.29 & $\mathrm{~F}$ & 24 & 0 & 0.24 & 0.0039 \\
\hline 51 & NGC7023 & -0.36 & II & 730 & $\mathrm{~F}$ & 0.032 & 0.031 \\
\hline 52 & NGC7023 & -0.62 & II & 920 & F & 0.76 & 0.13 \\
\hline 53 & L1172 & -0.55 & II & 210 & I & 0.53 & 0.43 \\
\hline 54 & L1228 S & -1.5 & II & 1600 & II & 0.015 & 0.011 \\
\hline 55 & NGC7023 & -1.5 & II & 2000 & II & 0.44 & 0.27 \\
\hline 56 & NGC7023 & -0.53 & II & 730 & $\mathrm{~F}$ & 0.057 & 0.057 \\
\hline 57 & NGC7023 & 0.51 & I & 500 & $\mathrm{~F}$ & 0.29 & 0.17 \\
\hline 58 & NGC7023 & -2.1 & III & 2000 & II & 0.16 & 0.11 \\
\hline 59 & NGC7023 & -1.00 & II & 1300 & II & 0.21 & 0.17 \\
\hline 60 & L1172 & -0.81 & II & 1200 & II & 3.3 & 2.1 \\
\hline 61 & Off-cloud & -0.97 & II & 1500 & II & 0.13 & 0.100 \\
\hline 62 & L1172 & -2.0 & III & 1800 & II & 45.0 & 37. \\
\hline 63 & L1221 & 0.84 & I & 66 & 0 & 3.0 & 0.73 \\
\hline 64 & L1221 & $\begin{array}{l}0.04 \\
1.0\end{array}$ & I & 21 & 0 & 1.4 & 0.0097 \\
\hline
\end{tabular}


Table 7

(Continued)

\begin{tabular}{|c|c|c|c|c|c|c|c|}
\hline Index & Region & $\begin{array}{l}\alpha_{\text {IR }} \\
\text { (a) } \\
\end{array}$ & $\begin{array}{c}\alpha_{\mathrm{IR}} \\
\text { Class }\end{array}$ & $\begin{array}{l}T_{\text {bol }} \\
(\mathrm{K}) \\
(\mathrm{b}) \\
\end{array}$ & $\begin{array}{c}T_{\text {bol }} \\
\text { Class } \\
\text { (c) }\end{array}$ & $\begin{array}{c}L_{\text {bol }} \\
\left(L_{\odot}\right) \\
(\mathrm{d})\end{array}$ & $\begin{array}{c}L_{\mathrm{IR}} \\
\left(L_{\odot}\right) \\
(\mathrm{e})\end{array}$ \\
\hline 65 & L1251W & 0.12 & F & 85 & I & 0.21 & 0.042 \\
\hline 66 & L1251W & 0.49 & I & 110 & I & 1.0 & 0.11 \\
\hline 67 & L1251W & 0.71 & I & 41 & 0 & 1.4 & 0.0012 \\
\hline 68 & L1251W & -0.51 & II & 38 & 0 & 0.68 & 0.0016 \\
\hline 69 & L1251A & -0.29 & $\mathrm{~F}$ & 400 & $\mathrm{~F}$ & 0.25 & 0.18 \\
\hline 70 & L1251A & -0.81 & II & 890 & $\mathrm{~F}$ & 0.11 & 0.081 \\
\hline 71 & L1251A & -0.23 & $\mathrm{~F}$ & 400 & $\mathrm{~F}$ & 0.17 & 0.13 \\
\hline 72 & L1251A & -0.54 & II & 720 & $\mathrm{~F}$ & 0.18 & 0.18 \\
\hline 73 & L1251A & -0.48 & II & 570 & $\mathrm{~F}$ & 0.22 & 0.18 \\
\hline 74 & L1251A & -0.85 & II & 930 & $\mathrm{~F}$ & 0.041 & 0.041 \\
\hline 75 & L1251A & 0.39 & I & 98 & I & 0.022 & 0.0087 \\
\hline 76 & L1251A & -0.83 & II & 1100 & II & 0.64 & 0.49 \\
\hline 77 & L1251A & -1.1 & II & 1100 & II & 0.77 & 0.71 \\
\hline 78 & L1251A & -0.28 & F & 610 & $\mathrm{~F}$ & 0.17 & 0.17 \\
\hline 79 & L1251A & -0.91 & II & 1200 & II & 0.30 & 0.25 \\
\hline 80 & L1251A & 0.033 & F & 410 & $\mathrm{~F}$ & 0.97 & 0.66 \\
\hline 81 & L1251A & -0.46 & II & 910 & $\mathrm{~F}$ & 0.48 & 0.43 \\
\hline 82 & L1251A & -1.1 & II & 1200 & II & 0.016 & 0.016 \\
\hline 83 & L1251B & -0.56 & II & 820 & $\mathrm{~F}$ & 0.40 & 0.20 \\
\hline 84 & L1251B & -0.44 & II & 510 & $\mathrm{~F}$ & 0.067 & 0.052 \\
\hline 85 & L1251B & -0.90 & II & 1000 & II & 0.099 & 0.077 \\
\hline 86 & L1251B & -0.69 & II & 790 & $\mathrm{~F}$ & 1.2 & 1.0 \\
\hline 87 & L1251B & -0.99 & II & 1400 & II & 0.088 & 0.071 \\
\hline 88 & L1251B & -0.96 & II & 980 & II & 0.076 & 0.056 \\
\hline 89 & L1251B & 1.2 & I & 48 & 0 & 3.3 & 0.075 \\
\hline 90 & L1251B & 1.6 & I & 50 & 0 & 15.0 & 0.99 \\
\hline 91 & L1251B & -1.1 & II & 1300 & II & 0.030 & 0.026 \\
\hline 92 & L1251B & 0.97 & I & 55 & 0 & 2.8 & 0.099 \\
\hline 93 & L1251B & -0.68 & II & 900 & $\mathrm{~F}$ & 0.010 & 0.010 \\
\hline 94 & L1251B & 0.94 & I & 310 & I & 0.64 & 0.50 \\
\hline 95 & L1251B & -2.1 & III & 2100 & II & 0.098 & 0.063 \\
\hline 96 & L1251B & -0.82 & II & 1100 & II & 0.42 & 0.29 \\
\hline 97 & L1251B & -0.72 & II & 770 & $\mathrm{~F}$ & 1.5 & 1.2 \\
\hline 98 & L1251B & -1.1 & II & 920 & $\mathrm{~F}$ & 0.087 & 0.087 \\
\hline 99 & Off-cloud & -1.3 & II & 2100 & II & 0.071 & 0.043 \\
\hline 100 & NGC7023 A & 0.14 & F & 27 & 0 & 0.16 & 0.0053 \\
\hline 101 & NGC7023 A & -0.95 & II & 1700 & II & 0.086 & 0.050 \\
\hline 102 & NGC7023 A & -1.4 & II & 1800 & II & 0.016 & 0.011 \\
\hline 103 & NGC7023 A & -1.3 & II & 1700 & II & 0.29 & 0.22 \\
\hline 104 & NGC7023 A & -0.052 & $\mathrm{~F}$ & 690 & $\mathrm{~F}$ & 0.039 & 0.039 \\
\hline 105 & L1221 & 1.0 & I & 640 & $\mathrm{~F}$ & 0.20 & 0.20 \\
\hline 106 & L1251C & -1.3 & II & 1500 & II & 0.014 & 0.012 \\
\hline 107 & L1251B & -1.7 & III & 2200 & II & 0.24 & 0.14 \\
\hline 108 & L1251B & -1.7 & III & 1600 & II & 0.068 & 0.056 \\
\hline 109 & L1251B & -0.69 & II & 1000 & II & 0.023 & 0.023 \\
\hline 110 & Off-cloud & -1.3 & II & 3300 & III & 0.091 & 0.069 \\
\hline 111 & Off-cloud & -0.016 & $\mathrm{~F}$ & 890 & $\mathrm{~F}$ & 0.070 & 0.061 \\
\hline 112 & Off-cloud & -1.9 & III & 4200 & III & 0.32 & 0.19 \\
\hline 113 & Off-cloud & -0.75 & II & 2100 & II & 0.054 & 0.047 \\
\hline 114 & L1147 & -1.2 & II & 1000 & II & 0.22 & 0.17 \\
\hline 115 & L1147 & -1.2 & II & 1600 & II & 0.012 & 0.0100 \\
\hline 116 & Off-cloud & -1.7 & III & 3500 & III & 0.17 & 0.068 \\
\hline 117 & Off-cloud & -2.1 & III & 2000 & II & 12.0 & 11. \\
\hline 118 & Off-cloud & -0.74 & II & 1400 & II & 0.049 & 0.049 \\
\hline 119 & Off-cloud & -1.8 & III & 2500 & II & 0.70 & 0.61 \\
\hline 120 & Off-cloud & -1.1 & II & 1800 & II & 0.88 & 0.75 \\
\hline 121 & Off-cloud & -1.0 & II & 2100 & II & 0.62 & 0.55 \\
\hline 122 & Off-cloud & -1.0 & II & 360 & $\mathrm{~F}$ & 0.070 & 0.067 \\
\hline 123 & L1228N & -0.89 & II & 1600 & II & 0.89 & 0.85 \\
\hline 124 & L1228 S & -0.93 & II & 1100 & II & 0.31 & 0.22 \\
\hline 125 & NGC7023 & -0.82 & II & 1100 & II & 0.68 & 0.51 \\
\hline 126 & Off-cloud & -1.2 & II & 1800 & II & 0.047 & 0.036 \\
\hline 127 & Off-cloud & -0.94 & II & 1500 & II & 0.34 & 0.30 \\
\hline 128 & Off-cloud & -1.0 & II & 1800 & II & 0.0070 & 0.0053 \\
\hline
\end{tabular}


Table 7

(Continued)

\begin{tabular}{|c|c|c|c|c|c|c|c|}
\hline Index & Region & $\begin{array}{l}\alpha_{\mathrm{IR}} \\
\text { (a) }\end{array}$ & $\begin{array}{c}\alpha_{\mathrm{IR}} \\
\text { Class }\end{array}$ & $\begin{array}{l}T_{\text {bol }} \\
(\mathrm{K}) \\
(\mathrm{b})\end{array}$ & $\begin{array}{c}T_{\text {bol }} \\
\text { Class } \\
\text { (c) }\end{array}$ & $\begin{array}{c}L_{\text {bol }} \\
\left(L_{\odot}\right) \\
(\mathrm{d})\end{array}$ & $\begin{array}{c}L_{\mathrm{IR}} \\
\left(L_{\odot}\right) \\
(\mathrm{e})\end{array}$ \\
\hline 129 & L1241 & -2.1 & III & 3000 & III & 2.1 & 1.6 \\
\hline 130 & Off-cloud & -1.4 & II & 2100 & II & 0.048 & 0.048 \\
\hline 131 & Off-cloud & -1.6 & II & 3300 & III & 0.11 & 0.082 \\
\hline 132 & Off-cloud & -1.0 & II & 1800 & II & 0.039 & 0.039 \\
\hline 133 & L1251C & -0.91 & II & 1600 & II & 0.14 & 0.13 \\
\hline 134 & L1157 & 0.94 & I & 37 & 0 & 8.9 & 0.021 \\
\hline 135 & Off-cloud & 0.66 & I & 100 & I & 46.0 & 23.0 \\
\hline 136 & L1228 S & -1.7 & III & 1200 & II & 0.0056 & 0.0056 \\
\hline 137 & NGC7023 A & -0.80 & II & 1400 & II & 0.23 & 0.20 \\
\hline 138 & NGC7023 A & -1.1 & II & 2400 & II & 100.0 & 43.0 \\
\hline 139 & NGC7023 A & -2.6 & III & 1900 & II & 0.27 & 0.27 \\
\hline 140 & L1228 S & $\ldots$ & $\ldots$ & $\ldots$ & $\ldots$ & & \\
\hline 141 & NGC7023 & $\ldots$ & $\ldots$ & $\ldots$ & $\ldots$ & & \\
\hline 142 & L1251A & -0.29 & $\mathrm{~F}$ & 430 & $\mathrm{~F}$ & 4.2 & 3.1 \\
\hline 143 & L1251A & 2.4 & I & 79 & I & 33.0 & 8.3 \\
\hline
\end{tabular}

Notes.

a The infrared spectral index, $\alpha_{\mathrm{IR}}$, calculated by a least-squares fit between 2 MASS $K_{s}$ and $24 \mu \mathrm{m}$. Where IRAC data were not available, $\alpha_{\mathrm{IR}}$ was calculated from the $K_{s}$-[24] color as described in Section 4.2.

b The bolometric temperature, $T_{\text {bol }}$, calculated using all available data from $B$ band to $1200 \mu \mathrm{m}$.

c The equivalent class calculated from $T_{\text {bol }}$ (see Section 5.2).

d The bolometric luminosity, $L_{\mathrm{bol}}$, calculated using all available data from $B$ band to $1200 \mu \mathrm{m}$.

e The infrared luminosity, $L_{\mathrm{IR}}$, calculated using all available data from 1 to $30 \mu \mathrm{m}$.

(This table is available in its entirety in a machine-readable form in the online journal. A portion is shown here for guidance regarding its form and content.)

Table 8

Class Statistics of Spitzer-identified YSOs

\begin{tabular}{lccc}
\hline \hline Class & & No. of YSOs & \\
& $\alpha_{\mathrm{IR}}$ & $T_{\text {bol }}$ & $\mathrm{c}^{\mathrm{a}}$ \\
\hline 0 & $\ldots$ & $17(13 \%)$ & $\ldots$ \\
I & $21(16 \%)$ & $11(8 \%)$ & $16 \%$ \\
"Flat" & $14(11 \%)$ & $38(29 \%)$ & $12 \%$ \\
II & $87(65 \%)$ & $62(47 \%)$ & $60 \%$ \\
III & $11(8 \%)$ & $5(4 \%)$ & $12 \%$ \\
\hline
\end{tabular}

Note.

a Total YSO statistics for all c2d regions (Evans et al. 2009).

of $\alpha_{\mathrm{IR}}$ classes for the Cepheus Flare with the values for other regions from the c $2 \mathrm{~d}$ survey (Figure 5 of Evans et al. 2009). The percentage of sources in each class closely matches the numbers for the entire c2d survey (shown in Column 4 of Table 8). Star formation in the Cepheus Flare is comprised of a series of small YSO groups, isolated cores, and a single (relatively) large YSO group (see Section 6). That this mixture gives the Cepheus Flare a relative number of sources equal to the c $2 \mathrm{~d}$ survey may just be a coincidence or it could be that the mixture of star-formation modes in Cepheus mirrors the balance of modes across the wider c2d sample.

Of the individual $\mathrm{c} 2 \mathrm{~d}$ regions, Cepheus most closely matches the $\alpha_{\mathrm{IR}}$ class profile of Serpens (Harvey et al. 2007), which is also very similar to that for the full c2d survey (Evans et al. 2009). Cepheus also has the same relative number of Class I sources as Serpens (Harvey et al. 2007). The relative number of Class I sources in Cepheus is $\sim 10$ percentage points higher than that seen in the Chamaeleon II and Lupus clouds although it is $\sim 5$ percentage points lower than that seen in the IC 5146 and Perseus clouds (Harvey et al. 2008; Evans et al. 2009).
Kun et al. (2008) include in their review of the Cepheus star-formation region a list of Classical and Weak-Lined $\mathrm{T}$ Tauri stars whose pre-main-sequence nature has been confirmed by spectroscopic observations. There are 31 Spitzer-identified YSOs from our sample that appear in that list (these have a reference to the Kun review in the last column of Table 6). Twenty-five of these sources (80\%) are classified as Class II by their $\alpha_{\mathrm{IR}}$ value. This match agrees with the usual interpretation of a YSO with a Class II infrared spectrum as most probably being a T Tauri protostar.

There are four sources on the Kun et al. list that are among the previously known YSOs listed in Table 16 that were coincident with our mapped area, but which were not identified as YSO candidates by the Spitzer schemes. Despite not being identified as YSOs, three of these four sources were coincident with entries in the Spitzer Cepheus catalog. Therefore, 34 out of 35 of the Kun et al. stars had infrared emission that was detectable by Spitzer. It should be noted, however, that the Kun et al. T Tauri list is from a project that is still underway and is not necessarily complete.

Our Class III to Class II ratio is $1 / 8$, whereas WTTS dominates CTTS in spatially complete surveys. For example, the WTTS/CTTS ratio in Taurus is 8 (Neuhaeuser et al. 1995) and $3 / 2$ in IC 348 (Luhman et al. 2003). Infrared selection schemes will naturally be less sensitive toward objects with smaller infrared excesses, i.e., Class III YSOs. The estimates of completeness can only be made if there is a previous census of WTTS to compare against. In a comparable study of the Lupus III region, Merín et al. (2008) estimated their completeness for Class III sources with infrared excesses against objects with no infrared excess as $\sim 50 \%$. If the low rate of $\mathrm{T}$ Tauri sources in the Cepheus cores without detectable infrared excesses is real and similar to Lupus III, it would further reinforce the idea that the YSO population is comparatively young. 

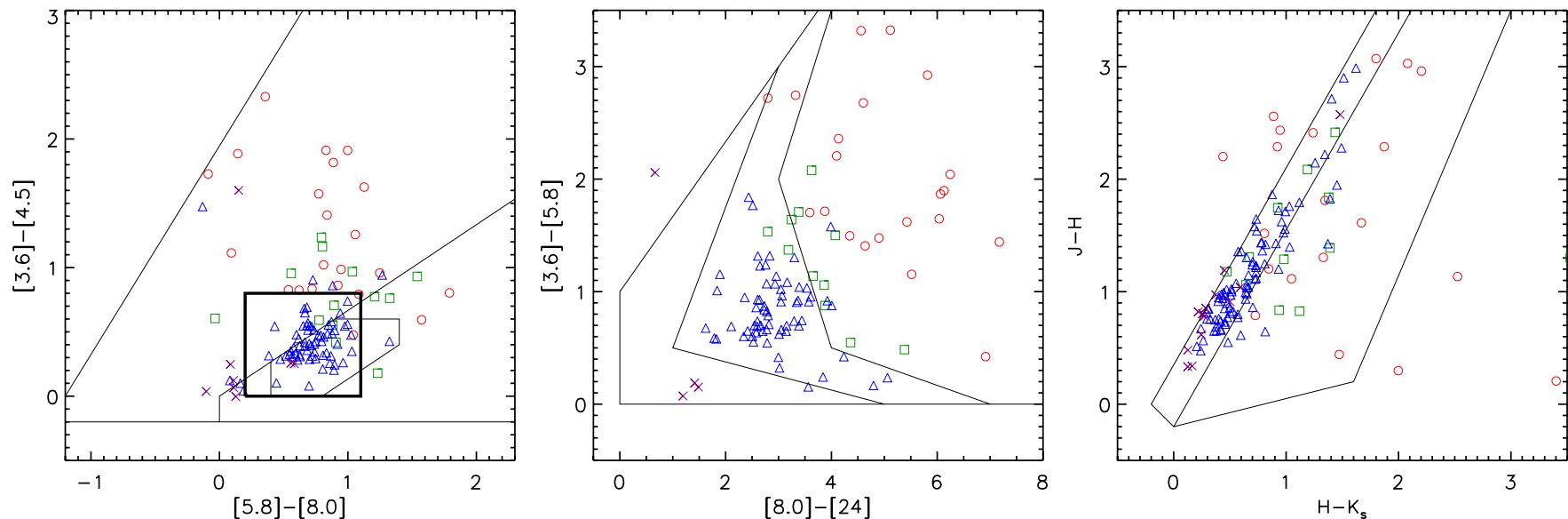

Figure 9. Color-color diagrams showing the correspondence between the colors of the YSO candidates and various color regions predicted by models of YSO spectra. The points are color coded to the YSOs $\alpha_{I R}$ classification—red are Class I sources, green are flat spectrum sources, blue are Class II sources, and purple are Class III sources. Approximate regions that contain the Robitaille models are shown on each panel by thin lines (Robitaille et al. 2006). The box with the thick outline plotted on the [5.8]-[8.0] vs. [3.6]-[4.5] plot is the region identified by Allen et al. (2004) as being the approximate domain of Class II sources. The three Robitaille regions on this plot show the region where any stage model can be found (bottom right), where the majority of Stage II models are found (small six-sided region), and where the majority of Stage I models can be found (top). The regions plotted on the [8.0]-[24] vs [3.6]-[5.8] plot approximate regions occupied by (from the left) Stage III, II, and I models. The box enclosing the majority of the sources in the $H-K_{s}$ vs. $J-H$ plot shows the region occupied by reddened stellar photospheres. The region to the right of this denotes the area where any evolution stage can occur.

(A color version of this figure is available in the online journal.)

Figure 7 shows that no 2MASS/MIPS YSO candidates are detected with a $K_{s}$-[24] color greater than $\sim 5$ (or 6). From Figure 8, we see that this means that the 2MASS/MIPS method is significantly biased toward the detection of Class II and Class III YSOs. Deeper $K_{s}$ data are needed to make a more complete survey of YSOs in the MIPS-only regions.

\subsection{Color-Color Diagrams}

Figure 9 shows color-color diagrams of the 143 Cepheus YSOs listed in Table 6. A source is included in a given plot if it has been detected at each of the wavelengths shown in that plot. Red markers show Class I sources, green markers show flat spectrum sources, blue markers show Class II sources, and purple markers show Class III sources. Robitaille et al. (2006) generated a database of 20,000 YSO models and used them to delineate a series of regions in the color-color space that correspond to three different phases of YSO evolution. They termed these evolutionary phases "Stages" to differentiate them from the equivalent "Classes" (a purely observational parameter that describes a source's infrared signature and may be influenced by reddening, e.g., as a result of the varying disk orientation). The approximate boundaries of the regions containing the different evolutionary states are shown in each of the three panels in Figure 9 (see Figure 23 of Robitaille et al. 2006).

Allen et al. (2004) compared the theoretical Spitzer colors of YSOs with data from the four embedded clusters. The region they identified as corresponding to the approximate domain of Class II sources contains almost all of our Class II candidates as shown by the thick lined box in the first panel of Figure 9 . The small six-sided region coincident with the Allen box shows the region where most of the Stage II Robitaille models lie. Our Class II sources cluster around this region, but there are a number of sources that lie just above it. These could be Class II sources that still retain some degree of reddening. The Class III sources are clustered around the zero point on each axis. Two large regions in the first panel are divided by a line that runs through the Stage II region and the Class II box. The region above this line contains the majority of the Robitaille Stage I models, whereas the region below it is where any Stage may be present. Our Class I sources agree quite well with the Stage I area.

The middle panel of Figure 9 shows IRAC and MIPS colors with the expected color space limits of the three Robitaille Stages (Stage III, II, and I running left to right). The points show the Cepheus YSO candidates with the same color code as the first panel. In this color space, there is also excellent agreement between the stage spaces and the classes. The green points show flat spectrum sources. These do not correspond to a specific stage, but are found along the Stage I/II boundary, as expected. Evans et al. (2009) found a similar result for the entire c2d survey and showed that the extinction correction of the individual fluxes only marginally improved the agreement between the classes and the stages. The stage regions take account of different angles of inclination, and this is partially why the Class I sources scatter over such a large area (Robitaille et al. 2006). The angle of inclination of a disk system to the observer is important as it can have a strong effect on the source's infrared signature and lead to the source being misclassified (Crapsi et al. 2008).

The last panel of Figure 9 shows the $H-K_{s}$ versus $J-H$ 2MASS colors of the Cepheus YSO candidates. The YSOs are not as separated as in the other two color spaces, but there is a trend for the Class I and Flat sources to scatter away from the main locus. The box around this locus shows the expected domain of reddened stellar photospheres from the Robitaille models, while the enclosed region to the right shows the region where any Stage evolutionary model can be present (Robitaille et al. 2006).

\section{CEPHEUS YSO PROPERTIES}

\subsection{Additional Photometry}

\subsubsection{Guide Star Catalog}

A search was made of the HST Guide Star Catalog-II (GSC-II) for visual companions within 5 arcsec of the Spitzer/ 2MASS sources. The GSC-II is an all-sky compilation of 
astrometric and photometric data from a series of different catalogs including the Palomar Sky Survey-II (POSS-II), the Palomar Quick-V survey, and Tycho Catalogs (McLean et al. 2000; Lasker et al. 2008). Of the 133 YSOs identified by Spitzer, 93 were also identified in the GSC-II. All of these had either "star" or "non-star" as their GSC-II spectral classification; none were classified as "galaxy."

Table 9 lists, where available, the GSC-II derived JohnsonCousins BVRI photometry for sources listed in Table 6. Column 1 lists the YSO candidates index from Table 6, Column 2 lists the GSC-II identifier of the associated source, and Columns 3-6 list the equivalent flux photometry in the $B V R I$ bands. The majority of the GSC-II identifications were from the POSS-II survey and had $B_{\mathrm{J}}, R_{\mathrm{F}}$, and $I_{\mathrm{N}}$ photometry. $R_{\mathrm{F}}$ and $I_{\mathrm{N}}$ photometry is from the Johnson-Cousins photometric system (Reid et al. 1991), $B_{\mathrm{J}}$ was converted to the same system using the Blair \& Gilmore (1982) color transforms. The data $V$ from the Palomar Quick-V survey were assumed to be in the standard Johnson system. The bright $\sim 7 \mathrm{mag} B_{\mathrm{T}}$ and $V_{\mathrm{T}}$ magnitudes for HD 200775 (YSO \#136) were from the Tycho survey and were converted to the Cousins-Johnson System (ESA 1997). All BVRI magnitudes were converted to fluxes using the Cousins photometric zero points (Bessell 1979). These zero points are listed in Column 4 of Table 3 and the transforms are summarized in Table 10. The errors on the optical photometry were typically $30 \%$ and could be as high as $50 \%$ in some cases.

\subsubsection{IRAS}

A search was also made for coincident sources from the IRAS Faint Source Catalog within 15 arcsec of the Spitzer positions (Moshir et al. 1990). This catalog was used as it gave more matches than the Point Source Catalog and may be more reliable in regions of strong nebulosity (Rebull et al. 2007). The lower resolution of the IRAS survey meant that it was possible to associate multiple Spitzer sources with a single IRAS source. In these cases, the IRAS source was assigned to the Spitzer source whose MIPS $24 \mu \mathrm{m}$ flux was nearest to the IRAS source's $25 \mu \mathrm{m}$ flux. No color corrections were applied to the IRAS fluxes. Column 7 of Table 9 lists the associated IRAS identifier and Columns 8-11 list the $12.5,25,60$, and $100 \mu \mathrm{m}$ fluxes and upper limits.

Twenty cross-identifications $(15 \%)$ were made between the 133 YSO candidates and the IRAS faint source catalog. Of these, nine are coincident with sources in the Kun (1998) list of IRASbased YSO candidates. Two sources are coincident with the Kun (1998) list, but are not cross-identified with IRAS in Table 9 because we restricted ourselves to just the Faint Source Catalog and did not include the Point Source Catalog. The Kun IRAS Sources are listed with literature names beginning with K98b (Kun 1998, Table 2) in column 3 of Table 6.

\subsubsection{Submillimeter}

While more evolved protostars may be discerned by a negative spectral slope or a high bolometric temperature, it is more difficult to determine the evolutionary state of young YSOs without examining emission from their envelopes at longer wavelengths. Table 11 lists the available far-infrared and submillimeter photometry for the Spitzer YSO candidates. The first column lists the YSO index and the second column lists the dark cloud associated with the YSO. Columns 3-6 list the actual fluxes quoted to a common precision. The last column lists, where relevant, a literature reference for the quoted flux. The $160 \mu \mathrm{m}$ fluxes were measured using a $40^{\prime \prime}$ radius aperture and the 450, 850, and $1200 \mu \mathrm{m}$ fluxes were measured in a $20^{\prime \prime}$ or $25^{\prime \prime}$ radius aperture depending on the quoted reference.

Column 3 of Table 11 lists the MIPS $160 \mu \mathrm{m}$ fluxes. The fluxes were measured from the unfiltered BCD maps. Backgrounds were subtracted using a sky annuli of 1.0-1.87 times the radius of the aperture. Several of the apertures contained more than one YSO candidate. For these sources, the measured flux was divided evenly between the candidates within the aperture. Several bright sources, including PV Cep and L1157, were found to be completely saturated in the $160 \mu \mathrm{m}$ BCD images. These sources and those within regions of large-scale extended emission where flux cannot be reasonably assigned to a compact YSO candidate have been excluded. The MIPS $160 \mu \mathrm{m}$ maps shown later are constructed from BCD images and have been filtered by a five-pixel diameter median filter to remove artifacts and to replace pixels excluded due to saturation.

Columns 4 and 5 of Table 11 list the SCUBA 450 and $850 \mu \mathrm{m}$ fluxes. The SCUBA on the JCMT was a submillimeter camera that could map the sky simultaneously at 850 and $450 \mu \mathrm{m}$. Fully sampled maps were produced by either scanning the hexagonal bolometer layout or offsetting the pointing center in a 64 point jiggle pattern (Holland et al. 1999).

Several of the $\mathrm{c} 2 \mathrm{~d}$ studies of the individual regions have existing SCUBA data for their sources. Young et al. (2006) undertook a targeted SCUBA campaign to observe cores in the c2d program. The cores they observed included L1157, L1221, L1228, L1251, and L1155C. The YSOs in L1251B are tightly clustered, making assignment of the lower resolution SCUBA flux difficult. In their analysis of L1251B, Lee et al. (2006) deconvolved the submillimeter emission of the embedded YSO candidates L1251B IRS 1, 2, and 4 by using the brightness ratio of the resolved $24 \mu \mathrm{m}$ sources. Their estimates are listed in Table 11. Data from Young et al. (2006) were used by the Young et al. (2009) study of L1221.

An additional search was made of the SCUBA archive for unpublished data coincident with other regions in this survey. Scan maps of NGC 7023 taken on 1999 October 16-17 and jiggle maps of PV Cep taken on 1997 October 16 were downloaded. The data were reduced in the normal manner using the SCUBA User Reduction Facility (Jenness \& Lightfoot 1998). The scan maps were restored using the Emerson 2 technique (Emerson 1995). The submillimeter zenith opacity at 850 and $450 \mu \mathrm{m}$ was determined using the "skydip" method and by comparison with the $1.3 \mathrm{~mm}$ sky opacity. Calibration was performed using observations of the planet Uranus taken during each shift. We estimate that the absolute calibration uncertainty is $\pm 10 \%$ at $850 \mu \mathrm{m}$ and $\pm 25 \%$ at $450 \mu \mathrm{m}$, based on the consistency and reproducibility of the calibration. Secondary beam-corrected photometry was performed in a $40^{\prime \prime}$ diameter aperture at the location of each YSO candidate.

Kauffmann et al. (2008) surveyed a selection of the c2d small cores with the $1.2 \mathrm{~mm}$ MAMBO bolometer array on the IRAM 30 m telescope (Kreysa et al. 1999). Seven of the regions they surveyed are coincident with the Cepheus Flare cores that contain YSO candidates. Photometry of these YSOs is listed in Column 6 of Table 11.

\subsection{Spectral Energy Distributions}

Figures 10, 11, 12 and 13 show plots of the SEDs of sources classified as Class I, Flat, Class II, and Class III, respectively. The open circles show, where available, the photometry from GSC-II, 2MASS, IRAC, MIPS, IRAS, and SCUBA. The arrows 
Table 9

Additional Fluxes

\begin{tabular}{|c|c|c|c|c|c|c|c|c|c|c|}
\hline Id. & GSC-II Name & $\begin{array}{c}B \\
(\mathrm{mJy})\end{array}$ & $\begin{array}{c}V \\
(\mathrm{mJy})\end{array}$ & $\begin{array}{c}R \\
(\mathrm{mJy})\end{array}$ & $\begin{array}{c}I \\
(\mathrm{mJy})\end{array}$ & IRAS Name & $\begin{array}{c}12.5(\mu \mathrm{m}) \\
(\mathrm{Jy})\end{array}$ & $\begin{array}{c}25(\mu \mathrm{m}) \\
(\mathrm{Jy})\end{array}$ & $\begin{array}{c}60(\mu \mathrm{m}) \\
(\mathrm{Jy})\end{array}$ & $\begin{array}{c}100(\mu \mathrm{m}) \\
(\mathrm{Jy})\end{array}$ \\
\hline 1 & & $\ldots$ & $\ldots$ & $\ldots$ & $\ldots$ & F20353+6742 & $<0.0522$ & 0.302 & 4.00 & 7.00 \\
\hline 2 & N1KR010841 & $\ldots$ & $\ldots$ & 0.109 & 0.641 & & $\ldots$ & $\ldots$ & $\ldots$ & $\ldots$ \\
\hline 3 & & $\ldots$ & $\ldots$ & $\ldots$ & $\ldots$ & F20358+6746 & 0.447 & 1.000 & 1.68 & $<5.71$ \\
\hline 4 & N1KP003099 & $\ldots$ & $\ldots$ & 0.0302 & 0.185 & & $\ldots$ & $\ldots$ & $\ldots$ & $\ldots$ \\
\hline 5 & & $\ldots$ & $\ldots$ & $\ldots$ & $\ldots$ & F20404+6712 & $<0.0636$ & 0.126 & 0.320 & $<4.39$ \\
\hline 6 & N0Y2007286 & $\ldots$ & $\ldots$ & 0.0872 & 0.715 & & $\ldots$ & $\ldots$ & $\ldots$ & $\ldots$ \\
\hline 7 & N0Y4008344 & $\ldots$ & $\ldots$ & 1.62 & 6.17 & & $\ldots$ & $\ldots$ & $\ldots$ & $\ldots$ \\
\hline 8 & N0Y4008341 & $\ldots$ & $\ldots$ & 0.0215 & $\ldots$ & & $\ldots$ & $\ldots$ & $\ldots$ & $\ldots$ \\
\hline 9 & N0Y4008297 & $\ldots$ & $\ldots$ & 0.0848 & 0.652 & F20582+7724 & 1.19 & 3.23 & 11.2 & 17.1 \\
\hline 11 & N0Y4008340 & $\ldots$ & $\ldots$ & 0.0283 & 0.324 & & $\ldots$ & $\ldots$ & $\ldots$ & $\ldots$ \\
\hline 12 & N0Y4012361 & $\ldots$ & $\ldots$ & 0.0497 & 0.190 & & $\ldots$ & $\ldots$ & $\ldots$ & $\ldots$ \\
\hline 13 & N0Y4008233 & $\ldots$ & $\ldots$ & 1.65 & 7.15 & & $\ldots$ & $\ldots$ & $\ldots$ & $\ldots$ \\
\hline 14 & N0Y4008023 & $\ldots$ & $\ldots$ & 0.0644 & 0.477 & & $\ldots$ & $\ldots$ & $\ldots$ & $\ldots$ \\
\hline 15 & N1KS012196 & 0.278 & 7.13 & 5.76 & 51.3 & $\mathrm{~F} 20587+6803$ & 0.558 & 0.952 & 0.822 & $<13.4$ \\
\hline 16 & N1KS010799 & $\ldots$ & 0.426 & 2.45 & 2.10 & & $\ldots$ & $\ldots$ & $\ldots$ & $\ldots$ \\
\hline 17 & & $\ldots$ & $\ldots$ & $\ldots$ & $\ldots$ & $F 20597+6801$ & $<0.136$ & 0.712 & $<7.33$ & $<88.6$ \\
\hline 20 & N1KS011770 & 0.00234 & 0.188 & 2.27 & 7.92 & & $\ldots$ & $\cdots$ & $\cdots$ & $\ldots$ \\
\hline 21 & N1KS009199 & 0.408 & 2.84 & 14.9 & 20.1 & & $\ldots$ & $\ldots$ & $\ldots$ & $\ldots$ \\
\hline 22 & N0Y4005594 & $\ldots$ & $\ldots$ & 0.0693 & 0.652 & & $\ldots$ & $\ldots$ & $\ldots$ & $\ldots$ \\
\hline 23 & N1KS011401 & $\ldots$ & 0.217 & 0.308 & 2.72 & & $\ldots$ & $\ldots$ & $\ldots$ & $\ldots$ \\
\hline 24 & N1KS026202 & $\ldots$ & $\ldots$ & $\ldots$ & 4.95 & & $\ldots$ & $\ldots$ & $\ldots$ & $\ldots$ \\
\hline 25 & N1KS011801 & $\ldots$ & 0.361 & 1.10 & 8.06 & & $\ldots$ & $\ldots$ & $\ldots$ & $\ldots$ \\
\hline 26 & N1KS011726 & $\ldots$ & $\ldots$ & 0.0245 & 0.143 & & $\ldots$ & $\ldots$ & $\ldots$ & $\ldots$ \\
\hline 27 & N1KS030305 & $\ldots$ & $\ldots$ & 0.0384 & $\ldots$ & & $\ldots$ & $\ldots$ & $\ldots$ & $\ldots$ \\
\hline 30 & N0Y4000288 & $\ldots$ & $\ldots$ & 52.1 & 97.8 & & $\ldots$ & $\ldots$ & $\ldots$ & $\ldots$ \\
\hline 31 & N1KS026411 & $\ldots$ & $\ldots$ & $\ldots$ & 0.293 & & $\ldots$ & $\ldots$ & $\ldots$ & $\ldots$ \\
\hline 32 & N0Y4004488 & $\ldots$ & $\ldots$ & 5.16 & 14.7 & $\mathrm{~F} 21023+7650$ & 0.171 & 0.225 & $<1.09$ & $<25.9$ \\
\hline 33 & N0Y4004376 & $\ldots$ & $\ldots$ & 5.45 & 14.0 & & $\ldots$ & $\ldots$ & $\ldots$ & $\ldots$ \\
\hline 35 & N0Y4004980 & $\ldots$ & $\ldots$ & 0.325 & 1.39 & & $\ldots$ & $\ldots$ & $\ldots$ & $\ldots$ \\
\hline 36 & N1KS011820 & $\ldots$ & 0.198 & 1.08 & 3.18 & & $\ldots$ & $\ldots$ & $\ldots$ & $\ldots$ \\
\hline 37 & N0Y4013403 & $\ldots$ & $\ldots$ & $\ldots$ & 0.145 & & $\ldots$ & $\ldots$ & $\ldots$ & $\ldots$ \\
\hline 38 & N1KS026404 & $\ldots$ & 1.25 & $\ldots$ & 10.2 & & $\ldots$ & $\ldots$ & $\ldots$ & $\ldots$ \\
\hline 39 & N1KS004876 & $\ldots$ & $\ldots$ & 0.0760 & 0.763 & & $\ldots$ & $\ldots$ & $\ldots$ & $\ldots$ \\
\hline 40 & N1KS011945 & $\ldots$ & 0.198 & 1.79 & 1.09 & & $\ldots$ & $\ldots$ & $\ldots$ & $\ldots$ \\
\hline 41 & N1KS009227 & $\ldots$ & 0.130 & 0.344 & 0.901 & & $\ldots$ & $\ldots$ & $\ldots$ & $\ldots$ \\
\hline 42 & N1KS026141 & $\ldots$ & $\ldots$ & $\ldots$ & 14.9 & & $\ldots$ & $\ldots$ & $\ldots$ & $\ldots$ \\
\hline 43 & N0Y4005414 & $\ldots$ & $\ldots$ & 3.02 & 16.5 & & $\ldots$ & $\ldots$ & $\ldots$ & $\ldots$ \\
\hline 45 & N1KS026373 & $\ldots$ & $\ldots$ & $\ldots$ & 5.09 & & $\ldots$ & $\ldots$ & $\ldots$ & $\ldots$ \\
\hline 46 & N0Y4000358 & $\ldots$ & $\ldots$ & 15.0 & 28.7 & F21028+7645 & 0.0847 & 0.198 & $<0.283$ & $<4.63$ \\
\hline 47 & N1KS017685 & $\ldots$ & $\ldots$ & 0.0462 & 0.272 & & $\ldots$ & $\ldots$ & $\ldots$ & $\ldots$ \\
\hline 48 & N1KS026025 & $\ldots$ & $\ldots$ & $\ldots$ & 0.183 & & $\ldots$ & $\ldots$ & $\ldots$ & $\ldots$ \\
\hline 49 & & $\ldots$ & $\ldots$ & $\ldots$ & $\ldots$ & $\mathrm{F} 21017+6742$ & $<0.136$ & 0.296 & 1.31 & 4.76 \\
\hline 51 & N1KS036074 & $\ldots$ & $\ldots$ & 0.0350 & $\ldots$ & & $\ldots$ & $\ldots$ & $\ldots$ & $\ldots$ \\
\hline 52 & N1KS008923 & 0.295 & 1.81 & 12.4 & 28.5 & & $\ldots$ & $\ldots$ & $\ldots$ & $\ldots$ \\
\hline 54 & N0Y4002847 & $\ldots$ & $\ldots$ & 0.126 & 0.821 & & $\ldots$ & $\ldots$ & $\ldots$ & $\ldots$ \\
\hline 55 & N1KS000226 & 0.307 & 4.67 & 15.7 & 22.0 & & $\ldots$ & $\ldots$ & $\ldots$ & $\ldots$ \\
\hline 57 & N1KS009855 & 0.313 & 3.00 & 5.60 & 4.81 & & $\ldots$ & $\ldots$ & $\ldots$ & $\ldots$ \\
\hline 58 & N1KS010406 & 0.0124 & 0.259 & 1.63 & 4.81 & & $\ldots$ & $\ldots$ & $\ldots$ & $\ldots$ \\
\hline 59 & N1KS010491 & 0.0276 & 0.388 & 2.17 & 3.69 & & $\ldots$ & $\ldots$ & $\ldots$ & $\ldots$ \\
\hline 60 & N1KS000328 & 53.4 & 48.0 & 108 & 49.0 & $\mathrm{~F} 21027+6747$ & 0.562 & 0.690 & $<0.520$ & $<4.98$ \\
\hline 61 & N1KS004279 & 0.140 & 0.338 & 2.45 & 3.55 & & $\ldots$ & $\ldots$ & $\ldots$ & $\ldots$ \\
\hline 62 & N1KS000600 & 1.16 & 12.2 & 44.1 & 533 & $\mathrm{~F} 21035+6730$ & 1.35 & 0.562 & $<0.315$ & $<7.01$ \\
\hline 66 & & $\ldots$ & $\ldots$ & $\ldots$ & $\ldots$ & $\mathrm{F} 22290+7458$ & $<0.0972$ & 0.333 & 0.774 & $<10.6$ \\
\hline 69 & N0XD010892 & $\ldots$ & $\ldots$ & 0.0234 & 0.239 & & $\ldots$ & $\ldots$ & $\ldots$ & $\ldots$ \\
\hline 70 & N0XD010042 & 0.00314 & 0.131 & 0.147 & 1.70 & & $\ldots$ & $\ldots$ & $\ldots$ & $\ldots$ \\
\hline 73 & N0XD022773 & $\ldots$ & $\ldots$ & 0.0271 & 0.176 & & $\ldots$ & $\ldots$ & $\ldots$ & $\ldots$ \\
\hline 76 & N0XD011103 & 0.332 & 1.86 & 1.45 & 15.8 & & $\ldots$ & $\ldots$ & $\ldots$ & $\ldots$ \\
\hline 77 & N0XD010877 & 0.0737 & 0.300 & 0.291 & 1.92 & & $\ldots$ & $\ldots$ & $\ldots$ & $\ldots$ \\
\hline 79 & N0XD010876 & 0.0162 & 0.348 & 0.576 & 5.04 & & $\ldots$ & $\ldots$ & $\ldots$ & $\ldots$ \\
\hline 80 & N0XD010918 & 0.142 & 0.754 & 2.81 & 13.3 & $\mathrm{~F} 22350+7502$ & 0.317 & 0.598 & $<7.05$ & $<71.8$ \\
\hline 81 & N0XD020969 & 0.00808 & 0.182 & $\ldots$ & 3.65 & & $\ldots$ & $\ldots$ & $\ldots$ & $\ldots$ \\
\hline 83 & N0XD006517 & 0.818 & 5.77 & 7.88 & 18.1 & F22367+7448 & $<0.0783$ & 0.132 & $<0.289$ & $<2.65$ \\
\hline 84 & N0XD023548 & $\ldots$ & $\ldots$ & $\ldots$ & 0.220 & & $\ldots$ & $\ldots$ & $\ldots$ & $\ldots$ \\
\hline 85 & N0XD007643 & 0.0361 & 0.195 & 0.350 & 1.61 & & $\ldots$ & $\ldots$ & $\ldots$ & $\ldots$ \\
\hline 86 & N0XD009088 & 0.0490 & 0.726 & 2.03 & 8.52 & $\mathrm{~F} 22372+7456$ & 0.178 & $<0.247$ & $<21.5$ & $<89.7$ \\
\hline 87 & N0XD009792 & 0.0116 & 0.140 & 0.194 & 1.67 & & $\ldots$ & $\ldots$ & $\ldots$ & $\ldots$ \\
\hline
\end{tabular}


Table 9

(Continued)

\begin{tabular}{|c|c|c|c|c|c|c|c|c|c|c|}
\hline Id. & GSC-II Name & $\begin{array}{c}B \\
(\mathrm{mJy}) \\
\end{array}$ & $\begin{array}{c}V \\
(\mathrm{mJy})\end{array}$ & $\begin{array}{c}R \\
(\mathrm{mJy}) \\
\end{array}$ & $\begin{array}{c}I \\
(\mathrm{mJy}) \\
\end{array}$ & IRAS Name & $\begin{array}{c}12.5(\mu \mathrm{m}) \\
(\mathrm{Jy})\end{array}$ & $\begin{array}{c}25(\mu \mathrm{m}) \\
(\mathrm{Jy})\end{array}$ & $\begin{array}{c}60(\mu \mathrm{m}) \\
(\mathrm{Jy})\end{array}$ & $\begin{array}{c}100(\mu \mathrm{m}) \\
(\mathrm{Jy})\end{array}$ \\
\hline 88 & N0XD008057 & 0.0101 & 0.162 & 0.187 & 1.41 & & $\ldots$ & $\ldots$ & $\ldots$ & \\
\hline 90 & & $\ldots$ & $\ldots$ & & & $\mathrm{F} 22376+7455$ & 0.693 & 5.47 & 31.1 & 67.6 \\
\hline 91 & N0XD008607 & $\ldots$ & $\ldots$ & 0.0205 & 0.285 & & $\ldots$ & $\ldots$ & $\ldots$ & $\ldots$ \\
\hline 92 & N0XD023537 & $\ldots$ & $\ldots$ & $\ldots$ & 0.277 & & $\ldots$ & $\ldots$ & $\ldots$ & $\ldots$ \\
\hline 95 & N0XD007466 & 0.0476 & 0.381 & 0.712 & 4.68 & & $\ldots$ & $\ldots$ & $\ldots$ & $\ldots$ \\
\hline 96 & N0XD008510 & 0.380 & 2.36 & 3.54 & 11.2 & & $\ldots$ & $\ldots$ & $\ldots$ & $\ldots$ \\
\hline 97 & N0XD009347 & 0.0598 & 1.68 & 5.76 & 19.3 & F22385+7457 & 0.309 & 0.330 & $<1.03$ & $<29.3$ \\
\hline 99 & N0XD008377 & 0.0445 & 0.627 & 0.535 & 3.86 & & $\ldots$ & $\ldots$ & $\ldots$ & $\ldots$ \\
\hline 101 & N1KS011801 & $\ldots$ & 0.361 & 1.10 & 8.06 & & $\ldots$ & $\ldots$ & $\ldots$ & $\ldots$ \\
\hline 102 & N1KS024073 & $\ldots$ & $\ldots$ & 0.0484 & 0.742 & & $\ldots$ & $\ldots$ & $\ldots$ & $\ldots$ \\
\hline 103 & N1KS026819 & $\ldots$ & $\ldots$ & $\ldots$ & 13.8 & & $\ldots$ & $\ldots$ & $\ldots$ & $\ldots$ \\
\hline 106 & N0XD023601 & $\ldots$ & $\ldots$ & $\ldots$ & 0.0917 & & $\ldots$ & $\ldots$ & $\ldots$ & $\ldots$ \\
\hline 107 & N0XD009004 & 0.343 & 2.21 & 4.88 & 14.0 & & $\ldots$ & $\ldots$ & $\ldots$ & $\ldots$ \\
\hline 108 & N0XD008908 & $\ldots$ & $\ldots$ & 0.157 & 0.978 & & $\ldots$ & $\ldots$ & $\ldots$ & $\ldots$ \\
\hline 110 & N1JU019152 & 0.242 & 1.01 & 1.60 & 1.81 & & $\ldots$ & $\ldots$ & $\ldots$ & $\ldots$ \\
\hline 111 & N1KP005099 & 0.383 & 0.512 & 0.525 & 0.696 & & $\ldots$ & $\ldots$ & $\ldots$ & $\ldots$ \\
\hline 112 & N1KP000227 & 3.09 & 6.62 & 9.74 & 10.4 & & $\ldots$ & $\ldots$ & $\ldots$ & $\ldots$ \\
\hline 113 & N1KR012754 & $\ldots$ & $\ldots$ & 0.930 & 0.735 & & $\ldots$ & $\ldots$ & $\ldots$ & $\ldots$ \\
\hline 114 & N1KP016685 & 0.424 & 1.16 & 2.49 & 4.56 & & $\ldots$ & $\ldots$ & $\ldots$ & $\ldots$ \\
\hline 115 & N1KP030229 & $\ldots$ & $\ldots$ & 0.0252 & 0.139 & & $\ldots$ & $\ldots$ & $\ldots$ & $\ldots$ \\
\hline 116 & N1KP010819 & 1.61 & 3.88 & 6.80 & 10.2 & & $\ldots$ & $\ldots$ & $\ldots$ & $\ldots$ \\
\hline 117 & N1KP012225 & $\ldots$ & 0.300 & 7.25 & $\ldots$ & F20436+6709 & 0.212 & 0.0681 & $<0.221$ & $<4.05$ \\
\hline 119 & N1KQ003034 & $\ldots$ & $\ldots$ & 5.71 & $\ldots$ & & $\ldots$ & $\ldots$ & $\ldots$ & $\ldots$ \\
\hline 120 & N1KQ008280 & 0.187 & 0.706 & 9.83 & 17.6 & & $\ldots$ & $\ldots$ & $\ldots$ & $\ldots$ \\
\hline 121 & N1KQ009201 & 0.0680 & 0.842 & 2.91 & 7.92 & & $\ldots$ & $\ldots$ & $\ldots$ & $\ldots$ \\
\hline 123 & N0Y4008425 & $\ldots$ & $\ldots$ & 2.52 & 7.92 & & $\ldots$ & $\ldots$ & $\ldots$ & $\ldots$ \\
\hline 124 & N0Y4000284 & $\ldots$ & $\ldots$ & 8.56 & 19.9 & F21016+7651 & $<0.0966$ & 0.149 & $<0.669$ & $<19.2$ \\
\hline 125 & N1KS019292 & 0.682 & 2.23 & 19.8 & 19.9 & $F 21017+6813$ & 0.119 & 0.140 & $<0.357$ & $<7.68$ \\
\hline 126 & N1KS019057 & $\ldots$ & $\ldots$ & 0.598 & 1.29 & & $\ldots$ & $\ldots$ & $\ldots$ & $\ldots$ \\
\hline 127 & N0Y4004915 & $\ldots$ & $\ldots$ & 3.23 & 9.69 & & $\ldots$ & $\ldots$ & $\ldots$ & $\ldots$ \\
\hline 128 & N0Y7009266 & $\ldots$ & $\ldots$ & 0.0598 & 0.390 & & $\ldots$ & $\ldots$ & $\ldots$ & $\ldots$ \\
\hline 129 & N0XW000215 & $\ldots$ & $\ldots$ & 46.6 & 73.5 & & $\ldots$ & $\ldots$ & $\ldots$ & $\ldots$ \\
\hline 131 & N0X6013952 & 0.196 & 1.48 & 1.79 & 2.85 & & $\ldots$ & $\ldots$ & $\ldots$ & $\ldots$ \\
\hline 133 & N0XD012039 & $\ldots$ & $\ldots$ & 0.0425 & 0.419 & & $\ldots$ & $\ldots$ & $\ldots$ & $\ldots$ \\
\hline 134 & & $\ldots$ & $\ldots$ & $\ldots$ & $\ldots$ & F20386+6751 & $<0.0656$ & 0.226 & 9.97 & 42.0 \\
\hline 135 & N1KR002065 & 0.0871 & 0.150 & 10.7 & 4.51 & F20453+6746 & 12.9 & 30.6 & 46.4 & 50.0 \\
\hline 137 & N1KS030305 & $\ldots$ & $\ldots$ & 0.0384 & $\ldots$ & & $\ldots$ & $\ldots$ & $\ldots$ & $\ldots$ \\
\hline 138 & N1KS000207 & 3430 & 3850 & $\ldots$ & $\ldots$ & & $\ldots$ & $\ldots$ & $\ldots$ & $\ldots$ \\
\hline 142 & N0XD000257 & 2.55 & 10.8 & 47.5 & 37.9 & F22331+7502 & 1.16 & 1.74 & 1.84 & $<21.9$ \\
\hline 143 & & $\ldots$ & $\ldots$ & $\ldots$ & $\ldots$ & F22343+7501 & 5.74 & 27.4 & 61.1 & 77.9 \\
\hline
\end{tabular}

(This table is available in its entirety in a machine-readable form in the online journal. A portion is shown here for guidance regarding its form and content.)

Table 10

Color-color Transforms to the Cousins-Johnson System

\begin{tabular}{lcc}
\hline \hline System & Transforms & Reference \\
\hline Quick & $V=V_{\text {Quick }}$ & \\
\hline Tycho & $V=V_{\mathrm{T}}-0.090\left(B_{\mathrm{T}}-V_{\mathrm{T}}\right)$ & The Hipparcos and Tycho \\
& $B=0.850\left(B_{\mathrm{T}}-V_{\mathrm{T}}\right)-V$ & Catalogs (ESA 1997) \\
\hline POSS-II & $B=\left(B_{\mathrm{J}}-0.28 V\right) / 0.72$ & Blair \& Gilmore 1982 \\
& $R=R_{\mathrm{F}}$ & \\
& $I=I_{\mathrm{N}}$ & \\
\hline
\end{tabular}

show the position of flux upper limits. The top left-hand corner of each plot is labeled with the index of the YSO. If the source index is followed by "x 0.01 ," it indicates that the SED has been scaled downward by two dex in order to place it on the same grid as the other SEDs. In Figures 12 and 13, the gray lines show two comparison SEDs that have been normalized near the peak of the dereddened SED (usually the 2MASS $J$ band). The solid gray line is a NEXTGEN profile for a K7 star (Hauschildt et al.
1999) and the dashed gray line with error bars is the median SED for a T Tauri star in Taurus (Hartmann et al. 2005). These SEDs are discussed in more detail in Section 5.4.

For the Class I YSOs in Figure 10 with three or more detections longward of $65 \mu \mathrm{m}$, we fit simple graybody SEDs assuming $\beta=2$ (see Kirk et al. 2007 for details) to characterize their submillimeter luminosities. Table 12 lists the derived parameters from these fits. Column 1 lists the YSO index, Column 2 lists the fitted dust temperature, and Column 3 lists the submillimeter luminosity integrated under the fitted graybody between $350 \mu \mathrm{m}$ and $2000 \mu \mathrm{m}$.

An alternative method to the spectral index for deriving the evolutionary classification of a YSO is to estimate the source's bolometric temperature, $T_{\text {bol }}$, which is the temperature of a blackbody that has the same mean frequency as a source's observed SED (Myers \& Ladd 1993). $T_{\text {bol }}$ can classify embedded protostars more effectively than the spectral index as it uses the entire spectrum and not just the infrared portion used for the spectral index (Enoch et al. 2009). Column 5 of Table 7 lists 
Table 11

MIPS $160 \mu \mathrm{m}$, SCUBA 450 and $850 \mu \mathrm{m}$, and MAMBO $1200 \mu \mathrm{m}$ Photometry of Cepheus YSO Candidates

\begin{tabular}{|c|c|c|c|c|c|c|}
\hline YSO \# & Region & $\begin{array}{c}160(\mu \mathrm{m}) \\
(\mathrm{Jy})\end{array}$ & $\begin{array}{c}450(\mu \mathrm{m}) \\
(\mathrm{Jy})\end{array}$ & $\begin{array}{c}850(\mu \mathrm{m}) \\
(\mathrm{Jy})\end{array}$ & $\begin{array}{c}1200(\mu \mathrm{m}) \\
(\mathrm{Jy})\end{array}$ & Ref. $^{\mathrm{a}}$ \\
\hline 1 & L1152 & $8.8 \pm 0.3$ & $8.3 \pm 4.4$ & $1.2 \pm 0.2$ & $\cdots$ & 1 \\
\hline 2 & L1152 & $1.6 \pm 0.1$ & $\cdots$ & $\cdots$ & $\cdots$ & \\
\hline 3 & L1152 & $1.6 \pm 0.1$ & $\ldots$ & $\ldots$ & $\ldots$ & \\
\hline 5 & L1148 & $\ldots$ & $\ldots$ & $\ldots$ & $0.059 \pm 0.007$ & 4 \\
\hline 7 & L1228N & $3.9 \pm 0.1$ & $\ldots$ & $\ldots$ & $\ldots$ & \\
\hline 8 & L1228N & $3.9 \pm 0.1$ & $\ldots$ & $\ldots$ & $\ldots$ & \\
\hline 9 & L1228N & $15 \pm 1$ & $1.1 \pm 1.4$ & $2.0 \pm 0.3$ & $0.89 \pm 0.01$ & 1,4 \\
\hline 17 & L1174 & $4.2 \pm 0.1$ & $\ldots$ & $\ldots$ & $\ldots$ & \\
\hline 18 & L1174 & $4.2 \pm 0.1$ & $\ldots$ & $\ldots$ & $\ldots$ & \\
\hline 34 & L1174 & $\ldots$ & $9.4 \pm 0.4$ & $1.4 \pm 0.1$ & $\ldots$ & \\
\hline 35 & L1228S & $6.2 \pm 0.3$ & $\ldots$ & $\ldots$ & $\ldots$ & \\
\hline 49 & L1172 & $1.7 \pm 0.1$ & $3.1 \pm 1.5$ & $0.35 \pm 0.07$ & $\ldots$ & 3 \\
\hline 50 & L1172 & $1.7 \pm 0.1$ & $\ldots$ & $0.32 \pm 0.06$ & $0.24 \pm 0.01$ & 3,4 \\
\hline 53 & L1172 & $1.7 \pm 0.1$ & $\ldots$ & $\ldots$ & $\ldots$ & \\
\hline 63 & L1221 & $\ldots$ & $10 \pm 6$ & $2.0 \pm 0.2$ & $\ldots$ & 1 \\
\hline 64 & L1221 & $\ldots$ & $11 \pm 6$ & $2.0 \pm 0.3$ & $\ldots$ & 1 \\
\hline 65 & L1251W & $\ldots$ & $\ldots$ & $\ldots$ & $0.35 \pm 0.01$ & 4 \\
\hline 66 & L1251W & $\ldots$ & $\ldots$ & $\ldots$ & $0.21 \pm 0.01$ & 4 \\
\hline 67 & L1251W & $\ldots$ & $\ldots$ & $\ldots$ & $0.46 \pm 0.01$ & 4 \\
\hline 68 & L1251W & $\ldots$ & $\ldots$ & $\ldots$ & $0.36 \pm 0.01$ & 4 \\
\hline $89^{\mathrm{b}}$ & L1251B & $\ldots$ & $2.1 \pm 1.1$ & $0.58 \pm 0.07$ & $\begin{array}{c}0.00+0.01 \\
\ldots\end{array}$ & 1,2 \\
\hline $90^{\mathrm{b}}$ & L1251B & $\ldots$ & $21 \pm 11$ & $5.8 \pm 0.7$ & $\ldots$ & 1,2 \\
\hline $92^{\mathrm{b}}$ & L1251B & $\ldots$ & $1.3 \pm 0.7$ & $0.34 \pm 0.04$ & $\ldots$ & 1,2 \\
\hline 100 & L1152 & $4.2 \pm 0.1$ & $\ldots$ & $\ldots$ & $\ldots$ & \\
\hline 134 & L1157 & $\ldots$ & $13 \pm 7$ & $2.5 \pm 0.5$ & $\ldots$ & 1 \\
\hline 135 & PV Cep & $\ldots$ & $12 \pm 1.0$ & $1.5 \pm 0.1$ & $\ldots$ & \\
\hline 139 & L1251A & $\ldots$ & $16 \pm 9$ & $2.3 \pm 0.3$ & $\ldots$ & 1 \\
\hline
\end{tabular}

\section{Notes.}

a The 850 and $450 \mu \mathrm{m}$ aperture radius was $20^{\prime \prime}$ for Young et al. (2006) and 25" for Visser et al. (2002). The Young et al. (2006) flux for L1251B was portioned between the sources by Lee et al. (2006) based on the relative brightness of the sources at $24 \mu \mathrm{m}$. The Kauffmann et al. (2008) $1200 \mu \mathrm{m}$ fluxes were measured in a $20^{\prime \prime}$ radius aperture. The $160 \mu \mathrm{m}$ fluxes were measured in a $40^{\prime \prime}$ radius aperture.

$\mathrm{b}$ The errors for these sources are taken as the same as the relative errors for the combined source, L1251E-1, from Young et al. (2006).

References. 1. Young et al. 2006; 2. Lee et al. 2006; 3. Visser et al. 2002; 4. Kauffmann et al. 2008.

Table 12

Class 0/I Derived Submillimeter Parameters

\begin{tabular}{lclc}
\hline \hline Id. & $\begin{array}{c}T_{\text {dust }} \\
(\mathrm{K})\end{array}$ & $\begin{array}{c}L_{\text {submm }} \\
\left(L_{\odot}\right)\end{array}$ & $\begin{array}{c}L_{\text {submm }} / L_{\text {bol }} \\
{[\%]}\end{array}$ \\
\hline 1 & 17 & 0.080 & 5.0 \\
49 & 15 & 0.054 & 9.4 \\
63 & 18 & 0.13 & 4.3 \\
64 & 17 & 0.13 & 9.1 \\
89 & 21 & 0.058 & 1.8 \\
90 & 17 & 0.48 & 3.1 \\
92 & 22 & 0.034 & 1.2 \\
134 & 18 & 0.25 & 2.8 \\
135 & 19 & 0.17 & 0.37 \\
143 & 19 & 0.23 & 0.69 \\
\hline
\end{tabular}

$T_{\text {bol }}$ for each protostar calculated using all available data points. A Class 0 source has $T_{\text {bol }}<70 \mathrm{~K}$, Class I has $T_{\text {bol }}=70-650 \mathrm{~K}$, Class II has $T_{\text {bol }}=650-2880 \mathrm{~K}$, and Class III has $T_{\text {bol }}>2880 \mathrm{~K}$ (Chen et al. 1995). The $T_{\text {bol }}$ boundaries for the equivalent of the intermediate flat spectrum class were not set by Chen et al. (1995), but Evans et al. (2009) recently suggested boundaries of $350 \mathrm{~K}$ and $950 \mathrm{~K}$. Column 6 of Table 7 lists the class for each YSO as derived from the Chen et al. (1995) boundaries with the Evans et al. (2009) modification for flat spectrum sources.

The number of YSOs in each class as classified by $T_{\text {bol }}$ is listed in column 3 of Table 8 . The spectral index cannot classify a source as a Class 0 source, so no total is listed the $\alpha_{\mathrm{IR}}$ column for such objects. While there is general agreement between the relative number of each class of YSOs between both schemes (i.e., a few Class III sources compared with many Class II sources), the bolometric method tends to skew a source's classification toward an earlier class (if the class changes at all). Out of the 87 sources classified as Class II by their value of $\alpha_{\mathrm{IR}}$, six (7\%) were classified as Class 0 or I by their value of $T_{\text {bol }}$. For example, YSO \#68 has a spectral index of -0.51 that classifies it as a Class II source, whereas it has a bolometric temperature of $38 \mathrm{~K}$ that places it firmly in the Class 0 regime. The spectral index is only calculated on flux data up to $24 \mu \mathrm{m}$, so flux data longward of $24 \mu \mathrm{m}$, as would be expected to dominate the SED of an embedded protostar, will not factor into a source's classification.

Conversely, out of the 62 sources classified as Class II by their value of $T_{\text {bol }}$, only YSO \#45 had a $T_{\text {bol }}$ Class earlier than Class II. As mentioned in Sections 4.2 and 4.3, the classification of a source by its $\alpha_{\mathrm{IR}}$ is sensitive to the angle of inclination of the circumstellar disk to the observer. Radiative transfer models have shown that Class II sources viewed at high angles of inclination can have SEDs that appear Class I like (Robitaille et al. 2006; Crapsi et al. 2008). The low frequency of this effect between the $\alpha_{\mathrm{IR}}$ and $T_{\text {bol }}$ classifications for the Class II and Class I sources would suggest either that the inclination angle effect is not significant in this sample or that the $T_{\text {bol }}$ classification is 


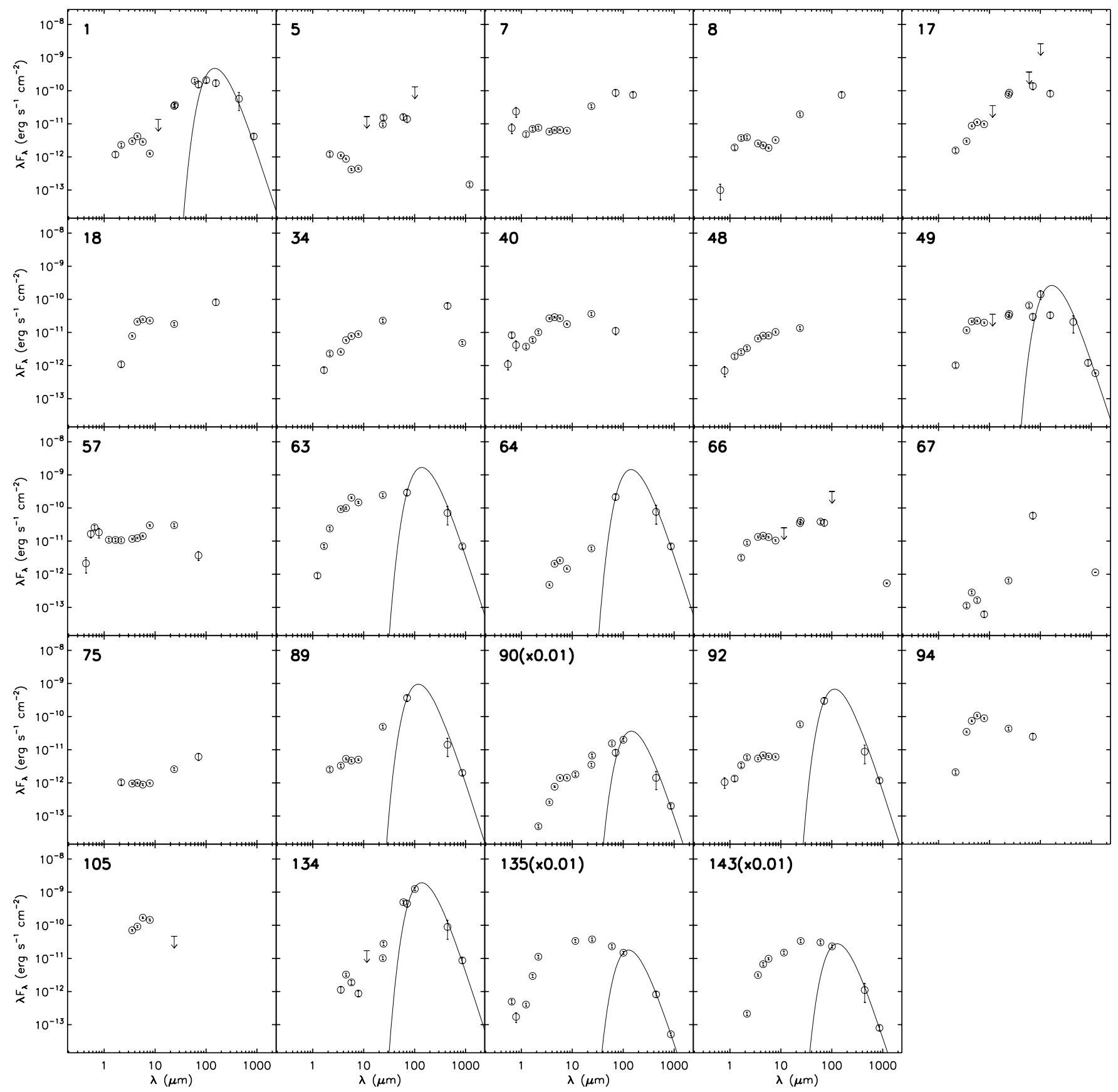

Figure 10. SEDs for Class I YSO candidates toward the Cepheus Flare. The open circles with error bars show the data points from GSC-II, 2MASS, IRAC, MIPS, $I R A S$, and SCUBA where available. Upper limits are also shown where available. The solid curve shows a simple graybody fit to the long wavelength data (see text for details). The YSO Id is shown in the top left-hand corner of each box. If this is followed by "x0.01," it indicates that the SED has been scaled downward by 2 dex in order to place it on the same grid as the other SEDs.

also effected by the inclination angle effect. In the latter case, the magnitude of the inclination angle effect could only be assessed by confirming the individual sources's evolutionary status with a spectrographic follow-up campaign.

Enoch et al. (2009) examined the effect on $T_{\text {bol }}$ of excluding a $160 \mu \mathrm{m}$ data point. They found that $T_{\mathrm{bol}}$ will be overestimated, and the ratio of Class 0 to Class I sources will be skewed toward Class I sources. For the Cepheus data, we found that the exclusion of all data longward of $150 \mu \mathrm{m}$ (i.e., the $160 \mu \mathrm{m}$ and submillimeter data listed in Table 11) did not significantly affect the numbers of sources classified as Flat or Class II and III. It did, however, affect the relative number of Class 0 and I sources. The number of Class 0 and I sources classified by $T_{\text {bol }}$ calculated using the submillimeter data is 17 and 11 , respectively (as shown in Table 8), whereas the number of Class 0 and I sources classified by $T_{\text {bol }}$ calculated without the submillimeter data is 4 and 16, respectively, in agreement with the Enoch et al. (2009) finding.

We retain the submillimeter data points when calculating the value of $T_{\text {bol }}$ quoted in Table 7 and used to calculate the statistics in Table 8 . The bolometric method undoubtedly gives a better assessment on the evolutionary status of an embedded protostar, and we discuss the individual values for potential Class 0 protostars in the Appendix. The number of sources 


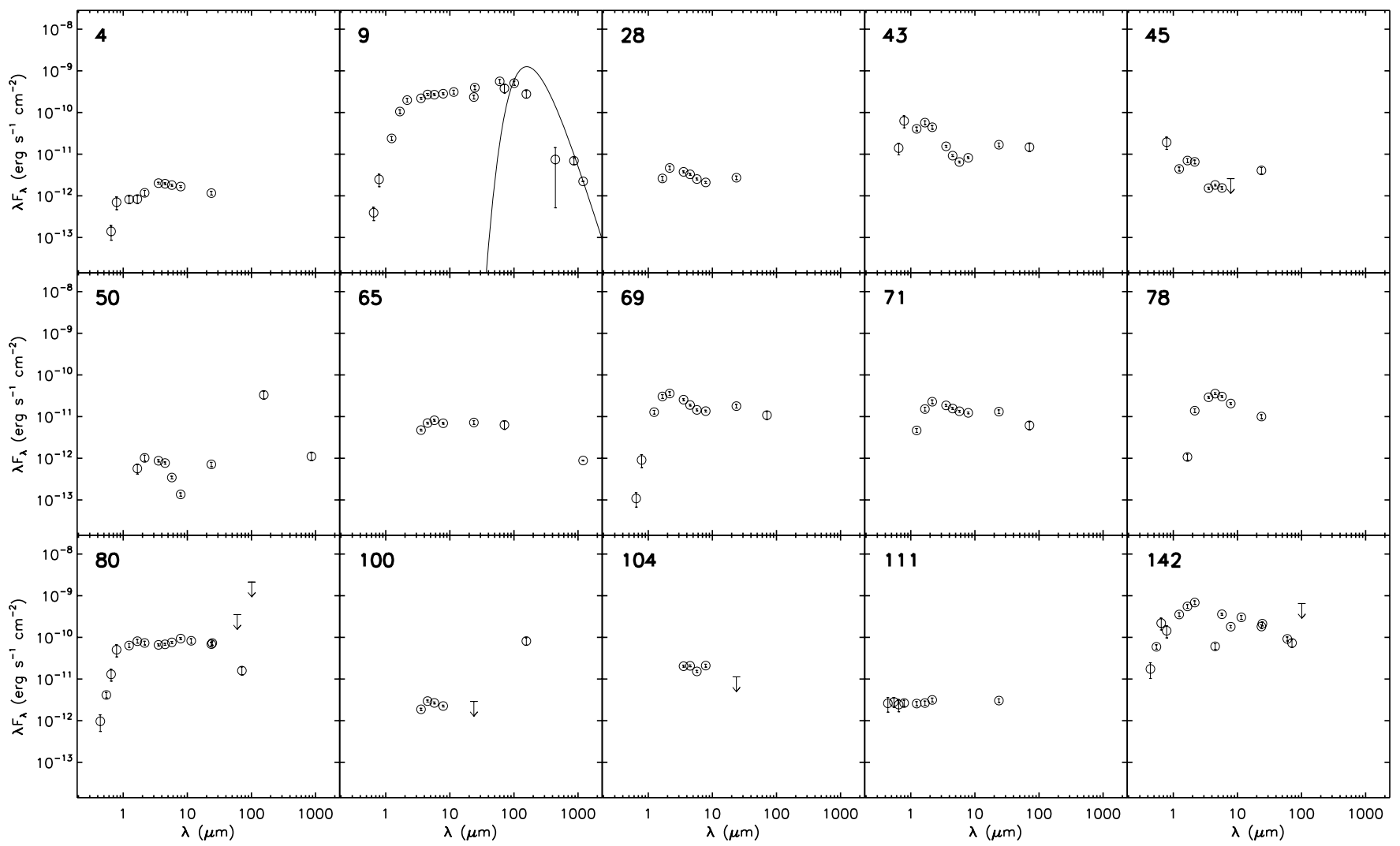

Figure 11. SEDs for flat spectral YSO candidates toward the Cepheus Flare. Details are the same as described in Figure 10.

with high-quality submillimeter data, however, is smaller than our total infrared sample size. Since this could bias our source classifications, we retain the $\alpha_{\mathrm{IR}}$ classifications as our main scheme. This is also done for consistency with earlier studies.

\subsection{Luminosity Function}

The bolometric luminosities, $L_{\mathrm{bol}}$, of the YSOs are estimated by integrating under all available data points in each YSO's SED using a simple trapezoidal method. The distance to each YSO was taken as the distance toward its respective associated dark cloud, as discussed in Section 2. The resulting luminosities are listed in Column 7 of Table 7. Yonekura et al. (1997) estimated the IRAS luminosity function of YSO sources associated with the molecular cores in their $\mathrm{CO}$ survey of Cepheus. They parameterized the number of sources $d N$ in the luminosity interval $L_{*}+d L_{*}$ as

$$
\frac{d N}{d L_{*}}=N_{0}\left(\frac{L_{*}}{L_{\odot}}\right)^{-p},
$$

where $N_{0}$ is a normalization factor and $p$ is the power-law index of the function. Yonekura et al. (1997) fit $N_{0}=8.9$ and $p=1.40 \pm 0.32$ above their completeness limit of $1 L_{\odot}$ for their close group of IRAS sources. Figure 14 shows in its upper panel the function for the 133 Spitzer-identified YSOs. The slopes were fitted by a least-squares fit above the estimated break of $\log \left(L_{*} / L_{\odot}\right)=-1.5$. The slope of the luminosity function fitted to all sources is $p=1.58 \pm 0.10$. This value agrees with the upper range of the Yonekura et al. (1997) error bars. The individual regions show a range of luminosities plus a single source that is approximately 1 dex more luminous than the rest of the YSO population.
The lower panel of Figure 14 shows the same histogram data as in the upper panel but plotted with $d N$ rather than $\log \left(d N / d\left(L / L_{\odot}\right)\right)$ on the abscissa. The luminosity distribution peaks at the point where the upper plot diverges from the single power law, i.e., at a luminosity of $0.06 L_{\odot}$. This Spitzer completeness limit is similar to that found for other $\mathrm{c} 2 \mathrm{~d}$ and Gould Belt regions (e.g., Harvey et al. 2007) and is 1.5 dex lower than the IRAS completeness limits (Yonekura et al. 1997).

A lot of sources will have a differing photometric coverage depending on the DSS, SCUBA, and IRAS detections. Therefore, we also calculate the more homogeneous $L_{\mathrm{IR}}$, the luminosity integrated between 1-30 $\mu \mathrm{m}$ for all sources with a $3.6 \mu \mathrm{m}$ detection. The distribution of $L_{\mathrm{IR}}$ is shown in Figure 14 as the dashed histogram, and the individual values are listed in Column 8 of Table 7 . There is good agreement in the peak position and width of the two histograms, but it is noticeable that the $L_{\mathrm{IR}}$ lacks $L_{\mathrm{bol}}$ 's higher luminosity tail. When compared with the equivalent $L_{\mathrm{IR}}$ distribution for Serpens and Lupus, the Cepheus $L_{\mathrm{IR}}$ distribution peaks in the same place, but the breadth of the peak is wider. All show the same peak just before $0.1 L_{\odot}$ followed by a sharp drop to zero around $0.01 L_{\odot}$ (Harvey et al. 2007; Merín et al. 2008).

\subsection{SED Modeling}

We conduct basic SED modeling in order to estimate the degree of circumstellar material around the Cepheus YSOs. The SED of a YSO that has been dereddened to remove attenuation from line-of-sight extinction will include contributions from several physical components. The dominant component at short wavelengths is a stellar component from the YSO itself. A second component from a lower temperature dusty circumstellar disk and envelope will appear as an excess to the stellar 


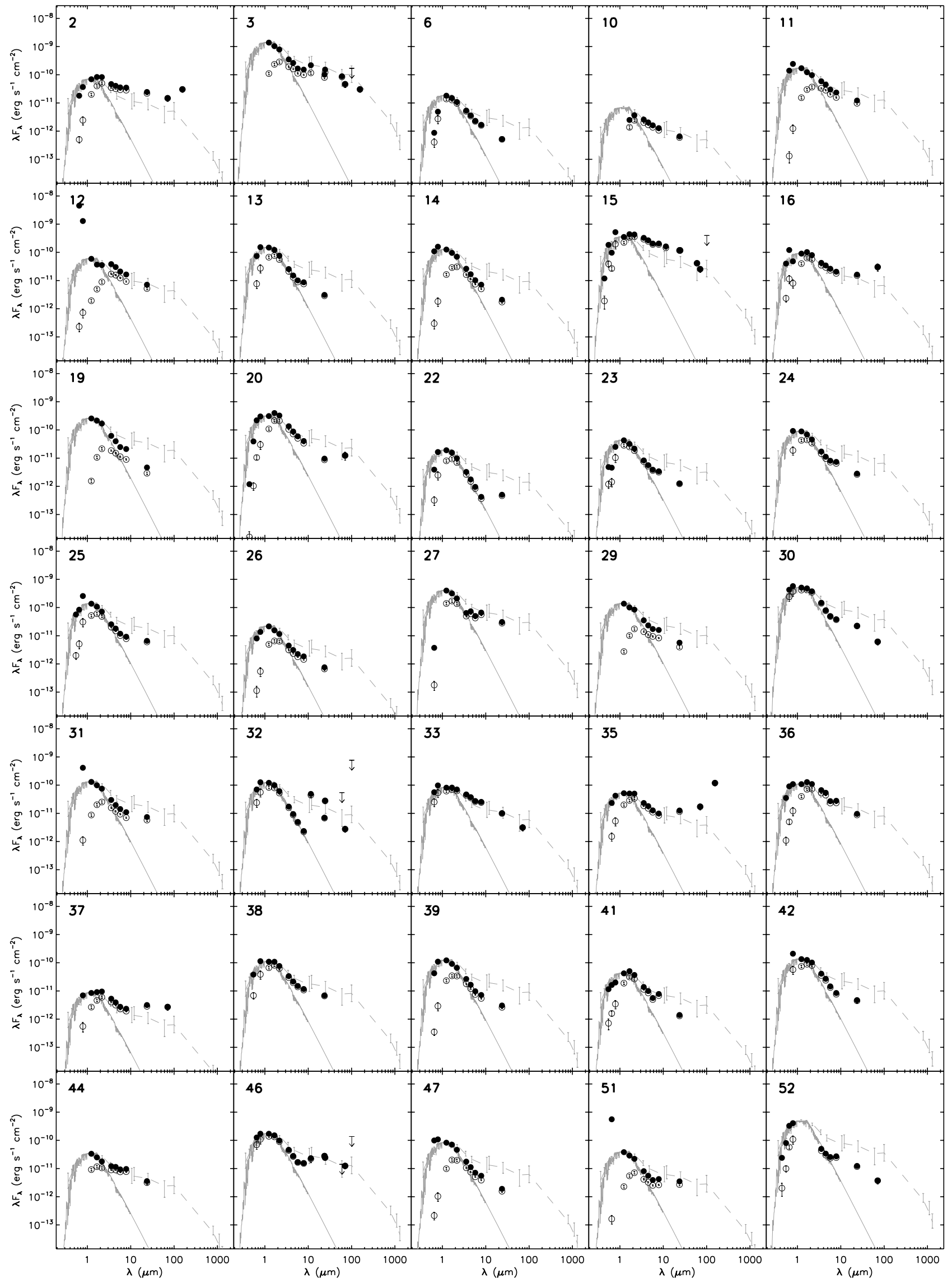

Figure 12. Class II candidate SEDs. Details are the same as described in Figure 10. Additionally, the filled circles show the dereddened data. The gray lines show two comparison SEDs that have been normalized near the peak of the dereddened SED (usually the 2MASS $J$ band). The solid gray line is a NEXTGEN profile for a K7 star (Hauschildt et al. 1999) and the dashed gray line with error bars is the median SED for a T Tauri star in Taurus (Hartmann et al. 2005). 


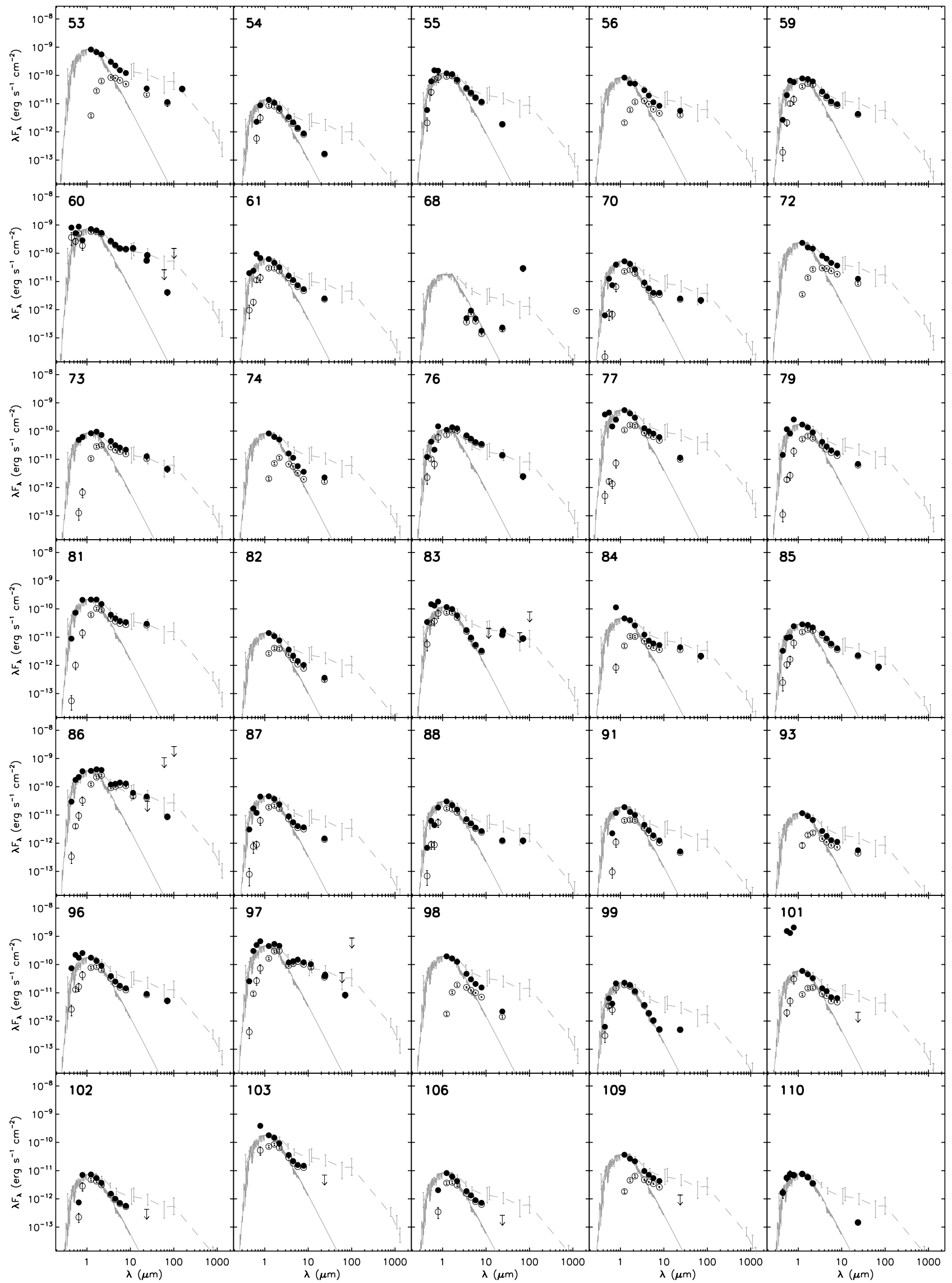

Figure 12. (Continued) 


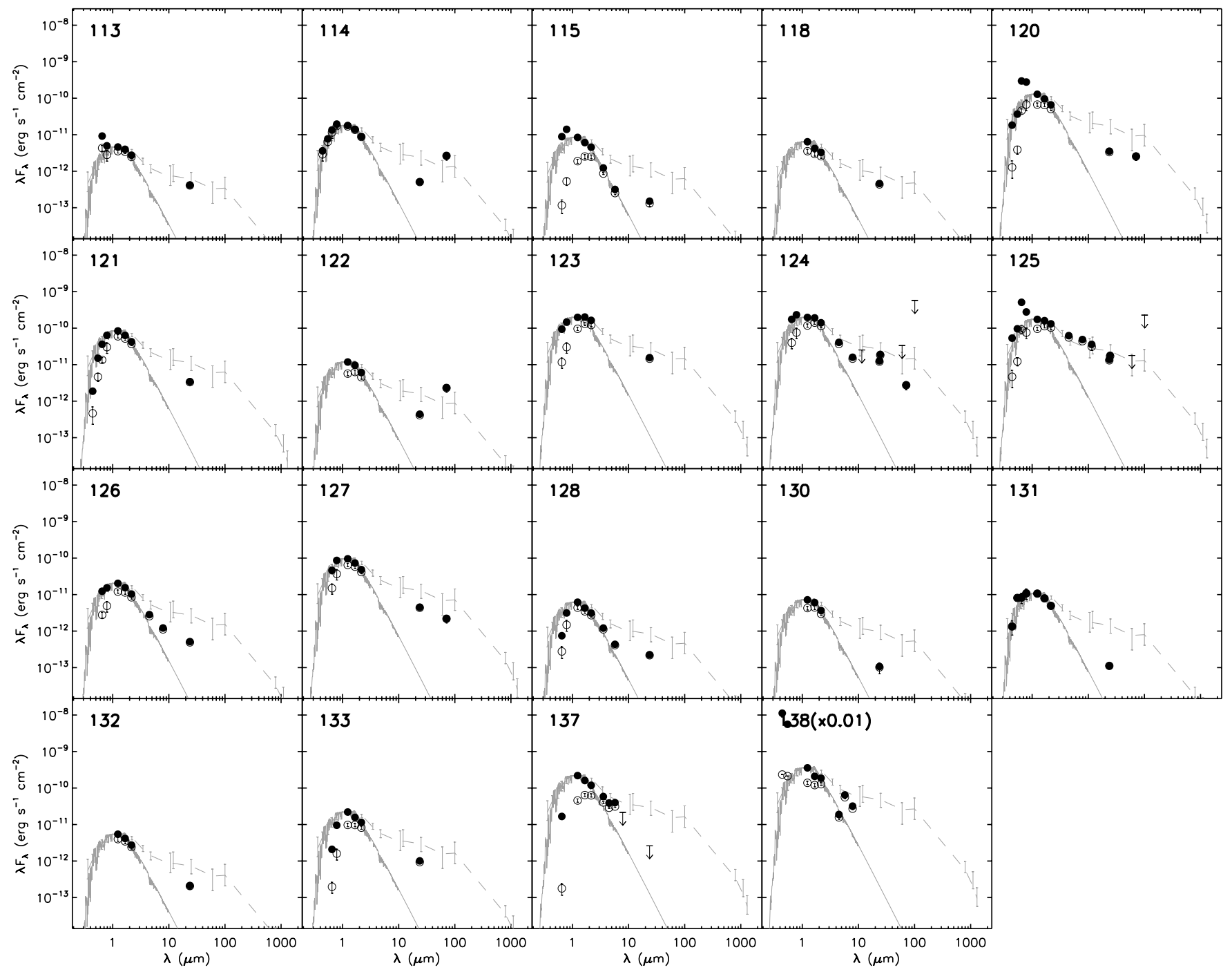

Figure 12. (Continued)

component at infrared and millimeter wavelengths. This infrared excess can be estimated if we first estimate the magnitude of the stellar component and subtract it from the dereddened SED. In order to do this, we need to know the amount of interstellar extinction between the YSO and the observer, and we need to know the underlying spectral type of the YSO.

The spectral type of the YSO needs to be known so that a model can be used to estimate the YSO's stellar SED component. In general this information is not available for the Spitzer-discovered YSOs since this requires a targeted campaign of spectroscopic observations, not available for Cepheus. We follow the approach taken by Harvey et al. $(2007,2008)$, who made the simplifying assumption that the underlying spectral type of most YSOs is a low-mass K7 star, or an intermediatemass A0 star in the case of the more luminous objects. This produced results statistically compatible with those obtained in Serpens, after an optical spectroscopic campaign had taken place (Oliveira et al. 2009).

For the Class II and Class III sources, the effect of reddening by line-of-sight extinction was removed from the SEDs by using the visual extinction toward each source calculated from its 2MASS $J-K_{S}$ color under the assumptions of the aforementioned K7 underlying spectral type and a $R=5.5$ interstellar extinction law (Weingartner \& Draine 2001). The dereddened data are shown in Figures 12 and 13 as solid markers. A NEXTGEN K7 profile (Hauschildt et al. 1999) normalized against the dereddened $J$ Band flux is plotted for comparison (the solid gray line). For a subset of sources, the dereddened optical photometry was significantly higher than the $\mathrm{K} 7$ profile that had been normalized to the dereddened $J$ band flux. These sources include the known variable stars FT Cep, FU Cep, EH Cep, and FV Cep. For these sources, it was necessary to modulate the estimated $A_{\mathrm{V}}$ down by a factor of 2-4 to make the $\mathrm{K} 7$ profile and the dereddened fluxes coincident.

The dashed gray line in Figures 12 and 13 is the median SED for a T Tauri star in Taurus (Hartmann et al. 2005) that has been normalized to the dereddened 2MASS $J$ flux. The profile represents a prototypical optically thick accretion disk surrounding a T Tauri star. The SEDs of some YSO candidates (e.g., YSO \#3) follow this profile quite closely, but others are closer to the $\mathrm{K} 7$ profile and only show a small amount of infrared excess at $24 \mu \mathrm{m}$ (e.g., YSO \#22). Approximately 30 YSOs (25\% of the disk population) have infrared excesses well below the median SED of the T Tauri stars in Taurus, implying that these sources have evolved or settled disks. This relatively low fraction also implies that the remaining disks in Cepheus show characteristics of being actively accreting and optically thick. 


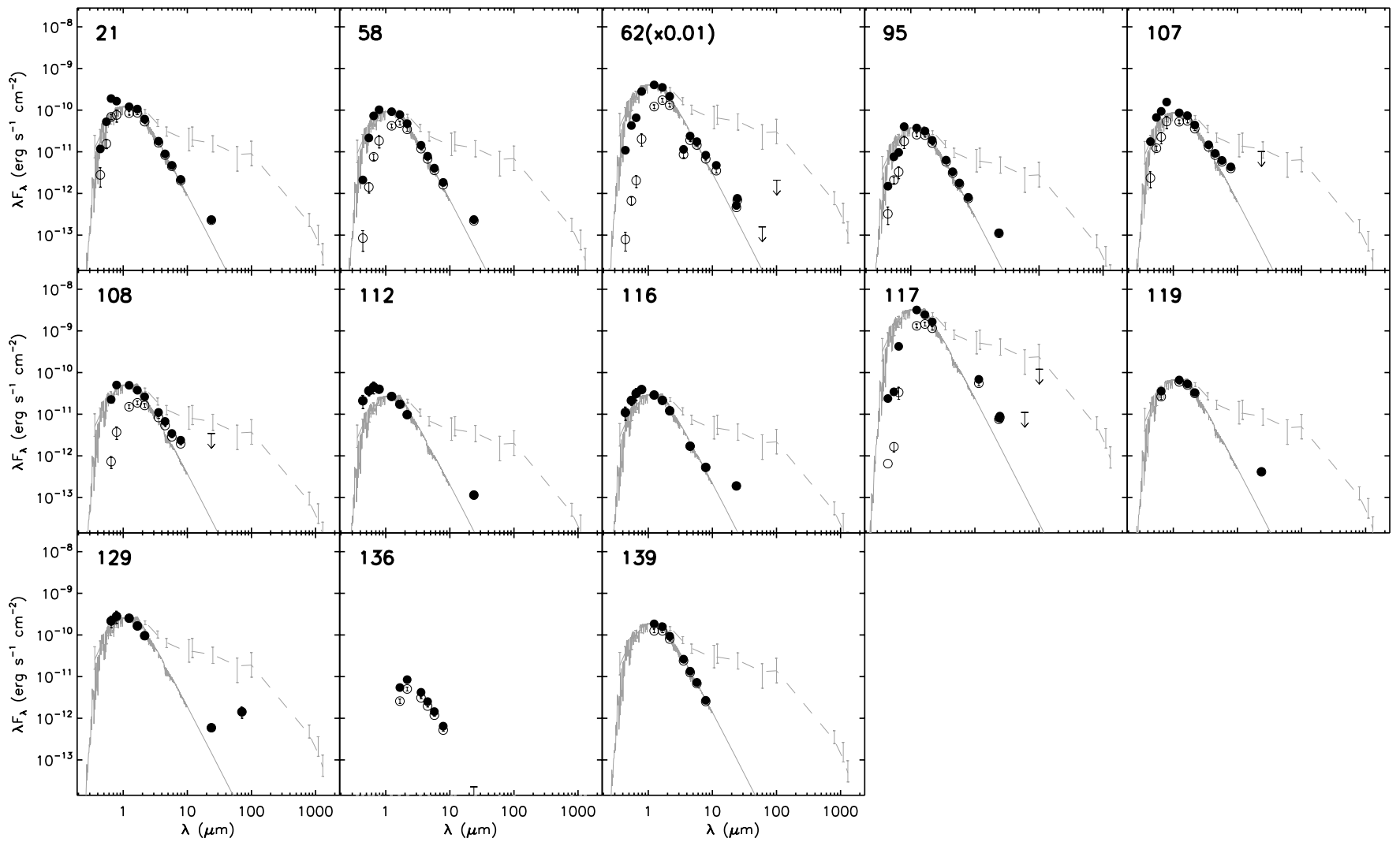

Figure 13. Class III candidate SEDs. Details are the same as described in Figure 12.

The amount of infrared excess can now be estimated for each YSO by subtracting the normalized K7 template (the solid gray line in Figures 12 and 13) from the dereddened SED data points (the filled circles in Figures 12 and 13). The luminosity of the YSO, $L_{\text {star }}$, was estimated by integrating the normalized stellar template after it had been interpolated to the observed wavelengths. The luminosity of the circumstellar disk, $L_{\text {disk }}$, was estimated by integrating the infrared excess at each of the observed wavelengths. Tables 13 and 14 list the SED modeling parameters and results for the Class II and Class III YSO candidates. Column 1 lists the YSO index. Column 2 lists the $A_{\mathrm{V}}$ that was used to deredden the SED and Column 3 lists the waveband that was used to normalize the stellar template. Columns 4 and 5 list $L_{\text {star }}$ and $\log \left(L_{\text {disk }} / L_{\text {star }}\right)$.

A histogram of $\log \left(L_{\text {disk }} / L_{\text {star }}\right)$ is shown in Figure 15. This ratio can be used to characterize a disk as either accretion, passive reprocessing, or debris-like (Kenyon \& Hartmann 1987; Hillenbrand et al. 2008). The dividing line between debris and passive disks suggested by Currie (2009) and used by earlier c2d papers was $\log \left(L_{\text {disk }} / L_{\text {star }}\right)=-1.7$; however, the Hillenbrand et al. (2008) study of young debris disks showed that their luminosities were well below the -1.7 value. We use a division of -3 based on the Hillenbrand et al. (2008) result. These divisions are marked in Figure 15.

The dashed histogram shown in Figure 15 only includes sources with complete IRAC photometry (i.e., it excludes the 2MASS/MIPS-identified sources). The difference between the two histograms shows that the majority of the sources identified as passive disks have been identified using the 2MASS/MIPS scheme. This pattern could be explained if $L_{\text {disk }}$ was underestimated due to the lack of IRAC photometry.
Assuming that the estimate of $L_{\text {star }}$ did not change, the addition of IRAC photometry could cause $L_{\text {disk }}$ to increase and would preferentially shift a source toward the accreting disk region. Thus, the observed frequency of passive disks could be lower than actually shown.

It is clear that the majority of the circumstellar disks modeled by this method are accretion disks with a peak in the distribution of $\log \left(L_{\text {disk }} / L_{\text {star }}\right)=0.4$. This is in agreement with the disk fractional luminosity for Lupus (Merín et al. 2008) and Chamaeleon (Alcalá et al. 2008). We do not, however, find as many passive and debris disks as was found in Serpens (Harvey et al. 2007). The differences in stellar ages and masses between clouds could account for different distributions, but a detailed analysis of this possibility is beyond the scope of this paper. The large percentage of accreting disks is consistent with our finding that a relatively small number of sources have infrared excesses below the median T Tauri SED in Taurus. Together, these points suggest that the disk average evolutionary status in Cepheus is very close to nascent and that there is little evidence for disk evolution.

Cieza et al. (2007) introduced the second-order parameters $\lambda_{\text {turnoff }}$ and $\alpha_{\text {excess }}$ to characterize the disk infrared excess. The first parameter, $\lambda_{\text {turnoff }}$, is the longest wavelength without a significant infrared excess. We compute this as the band shortward of the last band where the ratio of the disk flux to observed flux is greater than $80 \%$. When no band has a ratio greater than $80 \%$, we set $\lambda_{\text {turnoff }}$ equal to the longest wavelength band. The second parameter, $\alpha_{\text {excess }}$, is the spectral index of data points longward and inclusive of $\lambda_{\text {turnoff }}$ computed in the same manner as $\alpha_{\text {IR }}$. The calculated values of $\lambda_{\text {turnoff }}$ and $\alpha_{\text {excess }}$ for the Cepheus YSOs are listed in Columns 6 and 7 of Tables 13 and 14. Figure 16 shows a plot of $\lambda_{\text {turnoff }}$ versus $\alpha_{\text {excess }}$. The 
Table 13

SED Modeling Parameters for Class II YSOs

\begin{tabular}{|c|c|c|c|c|c|c|}
\hline Index & $A_{\mathrm{V}}$ & $\begin{array}{l}\lambda_{\text {norm }} \\
(\mu \mathrm{m})\end{array}$ & $\begin{array}{c}L_{\text {star }} \\
\left(L_{\odot}\right) \\
\end{array}$ & $\log \left(L_{\text {disc }} / L_{\text {star }}\right)$ & $\begin{array}{c}\lambda_{\text {turnoff }} \\
(\mu \mathrm{m})\end{array}$ & $\alpha_{\text {excess }}$ \\
\hline 2 & 2.3 & $J$ & 0.31 & 0.20 & 1.7 & -0.28 \\
\hline 3 & 9.5 & $J$ & 3.5 & -0.29 & 3.6 & -0.55 \\
\hline 6 & 0.50 & $J$ & 0.030 & -0.76 & 5.7 & -1.0 \\
\hline 10 & 3.8 & $K_{s}$ & 0.0040 & -0.045 & 3.6 & -0.70 \\
\hline 11 & 9.0 & $J$ & 0.29 & -0.38 & 2.2 & -0.87 \\
\hline 12 & 13.0 & $J$ & 0.099 & 1.1 & 2.2 & -0.74 \\
\hline 13 & 3.0 & $J$ & 0.25 & -0.93 & 5.7 & -0.86 \\
\hline 14 & 7.7 & $J$ & 0.21 & -0.67 & 3.6 & -1.3 \\
\hline 15 & 0.42 & $J$ & 1.3 & 0.16 & 2.2 & -0.72 \\
\hline 16 & 1.5 & $J$ & 0.33 & -0.084 & 2.2 & -0.26 \\
\hline 19 & 19.0 & $J$ & 0.52 & -0.58 & 1.7 & -1.5 \\
\hline 20 & 2.0 & $J$ & 1.2 & -0.31 & 2.2 & -0.97 \\
\hline 22 & 3.2 & $J$ & 0.032 & -1.5 & 7.9 & 0.16 \\
\hline 23 & 1.5 & $J$ & 0.16 & -1.0 & 5.7 & -0.81 \\
\hline 24 & 2.7 & $J$ & 0.28 & -0.80 & 5.7 & -0.77 \\
\hline 25 & 3.6 & $J$ & 0.50 & -0.44 & 5.7 & -0.38 \\
\hline 26 & 5.5 & $J$ & 0.075 & -0.93 & 3.6 & -0.89 \\
\hline 27 & 3.9 & $J$ & 1.3 & -0.70 & 3.6 & -0.41 \\
\hline 29 & 15.0 & $J$ & 0.28 & -0.49 & 2.2 & -1.1 \\
\hline 30 & 0.19 & $J$ & 0.85 & -0.49 & 5.7 & -0.80 \\
\hline 31 & 10.0 & $J$ & 0.41 & -0.15 & 3.6 & -0.67 \\
\hline 32 & 1.4 & $J$ & 0.20 & -0.34 & 7.9 & -0.35 \\
\hline 33 & 0.26 & $J$ & 0.14 & -0.078 & 3.6 & -0.87 \\
\hline 35 & 1.8 & $J$ & 0.087 & 0.15 & 2.2 & 0.18 \\
\hline 36 & 1.9 & $J$ & 0.40 & -0.17 & 2.2 & -1.0 \\
\hline 37 & 2.2 & $J$ & 0.013 & 0.096 & 1.7 & -0.32 \\
\hline 38 & 0.93 & $J$ & 0.40 & -0.55 & 3.6 & -0.76 \\
\hline 39 & 6.2 & $J$ & 0.42 & -1.00 & 3.6 & -1.1 \\
\hline 41 & 1.5 & $J$ & 0.16 & -0.43 & 3.6 & -1.1 \\
\hline 42 & 1.1 & $J$ & 0.42 & -0.41 & 3.6 & -1.1 \\
\hline 44 & 4.8 & $J$ & 0.068 & -0.14 & 2.2 & -0.64 \\
\hline 46 & 0.39 & $J$ & 0.29 & -0.43 & 5.7 & -0.043 \\
\hline 47 & 8.0 & $J$ & 0.29 & -0.57 & 3.6 & -1.1 \\
\hline 51 & 11.0 & $J$ & 0.13 & 0.71 & 3.6 & -0.35 \\
\hline 52 & 2.3 & I & 2.3 & -1.2 & 5.7 & -0.79 \\
\hline 53 & 20.0 & $J$ & 1.7 & -0.24 & 1.7 & -0.82 \\
\hline 54 & 1.8 & $J$ & 0.023 & -0.99 & 5.7 & -1.5 \\
\hline 55 & 0.48 & $J$ & 0.46 & -0.44 & 5.7 & -1.6 \\
\hline 56 & 14.0 & $J$ & 0.17 & -0.43 & 2.2 & -0.95 \\
\hline 59 & 1.2 & $J$ & 0.30 & -0.54 & 3.6 & -0.90 \\
\hline 60 & 0.18 & $J$ & 2.8 & -0.22 & 3.6 & -1.2 \\
\hline 61 & 2.8 & $J$ & 0.24 & -0.50 & 3.6 & -0.94 \\
\hline 68 & 5.1 & IRAC2 & 0.0029 & $\ldots$ & 7.9 & $\ldots$ \\
\hline 70 & 3.1 & $J$ & 0.22 & -0.91 & 5.7 & -0.26 \\
\hline 72 & 16.0 & $J$ & 0.51 & -0.31 & 2.2 & -1.0 \\
\hline 73 & 5.8 & $J$ & 0.32 & -0.15 & 1.7 & -0.76 \\
\hline 74 & 14.0 & $J$ & 0.18 & -0.83 & 3.6 & -0.99 \\
\hline 76 & 0.38 & $J$ & 0.46 & -0.030 & 2.2 & -1.1 \\
\hline 77 & 6.1 & $J$ & 2.3 & -0.63 & 3.6 & -1.3 \\
\hline 79 & 4.4 & $J$ & 0.71 & -0.47 & 3.6 & -0.90 \\
\hline 81 & 3.5 & $J$ & 0.89 & -0.51 & 3.6 & -0.33 \\
\hline 82 & 6.2 & $J$ & 0.031 & -0.70 & 3.6 & -1.1 \\
\hline 83 & 1.7 & $J$ & 0.49 & -0.29 & 7.9 & 0.47 \\
\hline 84 & 8.4 & $J$ & 0.16 & -0.17 & 3.6 & -0.47 \\
\hline 85 & 1.2 & $J$ & 0.12 & -0.41 & 2.2 & -0.89 \\
\hline 86 & 2.0 & $J$ & 1.5 & -0.19 & 3.6 & -0.90 \\
\hline 87 & 3.3 & $J$ & 0.19 & -0.90 & 5.7 & -0.74 \\
\hline 88 & 2.1 & $J$ & 0.13 & -0.80 & 5.7 & -0.43 \\
\hline 91 & 4.1 & $J$ & 0.072 & -0.98 & 3.6 & -1.1 \\
\hline 93 & 9.9 & $J$ & 0.025 & -0.60 & 3.6 & -0.77 \\
\hline 96 & 3.1 & $J$ & 0.73 & -0.32 & 5.7 & -0.47 \\
\hline 97 & 1.9 & $J$ & 1.9 & -0.10 & 3.6 & -0.90 \\
\hline 98 & 18.0 & $J$ & 0.43 & -0.60 & 1.7 & -1.6 \\
\hline 99 & 0.66 & $J$ & 0.095 & -1.3 & 7.9 & -0.027 \\
\hline 101 & 7.2 & $J$ & 0.22 & 1.1 & 3.6 & -1.1 \\
\hline
\end{tabular}


Table 13

(Continued)

\begin{tabular}{|c|c|c|c|c|c|c|}
\hline Index & $A_{\mathrm{V}}$ & $\begin{array}{l}\lambda_{\text {norm }} \\
(\mu \mathrm{m}) \\
\end{array}$ & $\begin{array}{l}L_{\text {star }} \\
\left(L_{\odot}\right) \\
\end{array}$ & $\log \left(L_{\text {disc }} / L_{\text {star }}\right)$ & $\begin{array}{c}\lambda_{\text {turnoff }} \\
(\mu \mathrm{m})\end{array}$ & $\alpha_{\text {excess }}$ \\
\hline 102 & 1.6 & $J$ & 0.025 & -1.1 & 5.7 & -0.72 \\
\hline 103 & 3.4 & $J$ & 0.55 & -0.44 & 5.7 & -0.20 \\
\hline 106 & 3.0 & $J$ & 0.027 & -1.1 & 5.7 & -0.67 \\
\hline 109 & 11.0 & $J$ & 0.079 & -0.75 & 2.2 & -1.3 \\
\hline 110 & 0.00 & $J$ & 0.099 & -1.4 & 2.2 & -1.3 \\
\hline 113 & 0.49 & $J$ & 0.058 & -0.86 & 2.2 & -0.79 \\
\hline 114 & 0.20 & $J$ & 0.23 & -1.2 & 2.2 & -0.48 \\
\hline 115 & 5.6 & $J$ & 0.039 & -0.59 & 5.7 & -0.52 \\
\hline 118 & 2.2 & $J$ & 0.068 & -2.0 & 2.2 & -0.82 \\
\hline 120 & 2.4 & $J$ & 1.3 & -0.66 & 2.2 & -0.98 \\
\hline 121 & 1.3 & $J$ & 0.86 & -2.3 & 2.2 & -1.1 \\
\hline 122 & 2.7 & $J$ & 0.099 & -1.4 & 2.2 & -0.41 \\
\hline 123 & 1.4 & $J$ & 0.94 & -0.42 & 2.2 & -0.98 \\
\hline 124 & 0.96 & $J$ & 0.35 & -0.41 & 2.2 & -0.95 \\
\hline 125 & 1.1 & $J$ & 0.70 & -0.059 & 2.2 & -0.86 \\
\hline 126 & 1.9 & $J$ & 0.075 & -1.1 & 4.5 & -0.99 \\
\hline 127 & 1.5 & $J$ & 0.45 & -1.6 & 2.2 & -0.90 \\
\hline 128 & 1.3 & $J$ & 0.011 & -1.2 & 5.7 & -0.46 \\
\hline 130 & 2.0 & $J$ & 0.065 & -2.7 & 2.2 & -1.5 \\
\hline 131 & 0.00 & $J$ & 0.12 & -1.3 & 2.2 & -1.6 \\
\hline 132 & 1.2 & $J$ & 0.049 & -2.3 & 2.2 & -1.1 \\
\hline 133 & 3.1 & $J$ & 0.23 & -2.3 & 2.2 & -1.0 \\
\hline 137 & 5.9 & $K_{S}$ & 0.72 & -1.1 & 3.6 & -0.80 \\
\hline 138 & 3.5 & $J$ & 130 & 1.0 & 4.5 & 0.78 \\
\hline
\end{tabular}

Table 14

SED Modeling Parameters for Class III YSOs

\begin{tabular}{|c|c|c|c|c|c|c|}
\hline Index & $A_{\mathrm{V}}$ & $\begin{array}{l}\lambda_{\text {norm }} \\
(\mu \mathrm{m})\end{array}$ & $\begin{array}{l}L_{\text {star }} \\
\left(L_{\odot}\right)\end{array}$ & $\log \left(L_{\text {disc }} / L_{\text {star }}\right)$ & $\begin{array}{c}\lambda_{\text {turnoff }} \\
(\mu \mathrm{m})\end{array}$ & $\alpha_{\text {excess }}$ \\
\hline 21 & 1.3 & $J$ & 0.46 & -0.57 & 24.0 & $\cdots$ \\
\hline 58 & 2.9 & $J$ & 0.35 & -0.99 & 7.9 & $\cdots$ \\
\hline 62 & 4.5 & $J$ & 150.0 & -2.1 & 25.0 & $\ldots$ \\
\hline 95 & 1.4 & $J$ & 0.16 & -1.1 & 7.9 & $\cdots$ \\
\hline 107 & 1.8 & $J$ & 0.36 & -0.41 & 7.9 & $\ldots$ \\
\hline 108 & 4.5 & $J$ & 0.19 & -1.0 & 5.7 & $\ldots$ \\
\hline 112 & 0.00 & $J$ & 0.35 & -0.75 & 2.2 & $\cdots$ \\
\hline 116 & 0.00 & $J$ & 0.15 & -0.62 & 7.9 & $\cdots$ \\
\hline 117 & 3.3 & $J$ & 25.0 & -2.2 & 2.2 & $\ldots$ \\
\hline 119 & 0.39 & $J$ & 0.82 & -3.2 & 2.2 & $\ldots$ \\
\hline 129 & 0.00 & $J$ & 2.7 & -1.5 & 24.0 & 0.80 \\
\hline 136 & 4.8 & $J$ & $\ldots$ & $\ldots$ & 1.7 & $\ldots$ \\
\hline 139 & 1.4 & $J$ & 0.37 & -2.1 & 7.9 & $\ldots$ \\
\hline
\end{tabular}

Class II YSOs are shown by open circles and Class III YSOs are shown by asterisks.

Cieza et al. (2007) found an evolutionary sequence along $\lambda_{\text {excess }}$ with Classical T Tauri stars having a $\lambda_{\text {excess }}$ shortward of 2MASS $K_{s}$. They also showed that the majority of $\alpha_{\text {excess }}$ values cluster around -1 irrespective of $\lambda_{\text {turnoff }}$, but that the spread in $\alpha_{\text {excess }}$ increased with $\lambda_{\text {turnoff }}$. Figure 16 shows the same trend for clustering around $\alpha_{\text {excess }} \sim-1$, but as most stars have had their stellar profiles normalized to the 2MASS $J$ we would not expect to see values of $\lambda_{\text {turnoff }}$ equal to it. Surprisingly, there are no objects with $\lambda_{\text {turnoff }}=4.5 \mu \mathrm{m}$. The calculation of $\alpha_{\text {excess }}$ and $\lambda_{\text {turnoff }}$ is affected by the normalization of the stellar profile and the assumed spectral type. Thus, the empty $4.5 \mu \mathrm{m}$ band and the scatter of excesses at shorter wavelengths could possibly be due to imperfect knowledge of each source's spectral type.

Harvey et al. (2007) plotted a similar diagram for Serpens and showed that the Serpens Class III sources predominately had a $\lambda_{\text {turnoff }} \geqslant 5.8 \mu \mathrm{m}$. We find a similar result for the Class III sources in Cepheus as shown by the values of $\lambda_{\text {turnoff }}$ in column 6 of Table 14 and the few sources that appear at IRAC 3 and 4 in Figure 16. We are unable to calculate $\alpha_{\text {excess }}$ for the three sources with $\lambda_{\text {turnoff }}=24 \mu \mathrm{m}$ as none of these sources have longer wavelength data points against which to calculate an index. All three were Class III sources, however, and would have followed the trend for those sources appearing to the right in Figure 16.

Merín et al. (2008) showed that an object in the top right of the diagram was likely to have a transitional disk - an optically thick disk with a central cavity larger than several AU. These objects appear as a photospheric SED with an infrared excess only at the longest wavelengths. The object in the top right of Figure 16 is YSO \#83, a Class II YSO in L1251B associated with IRAS F22367+7448. It has been detected in X-rays and was classified as a Classical T Tauri based on its large $\mathrm{H} \alpha$ equivalent 


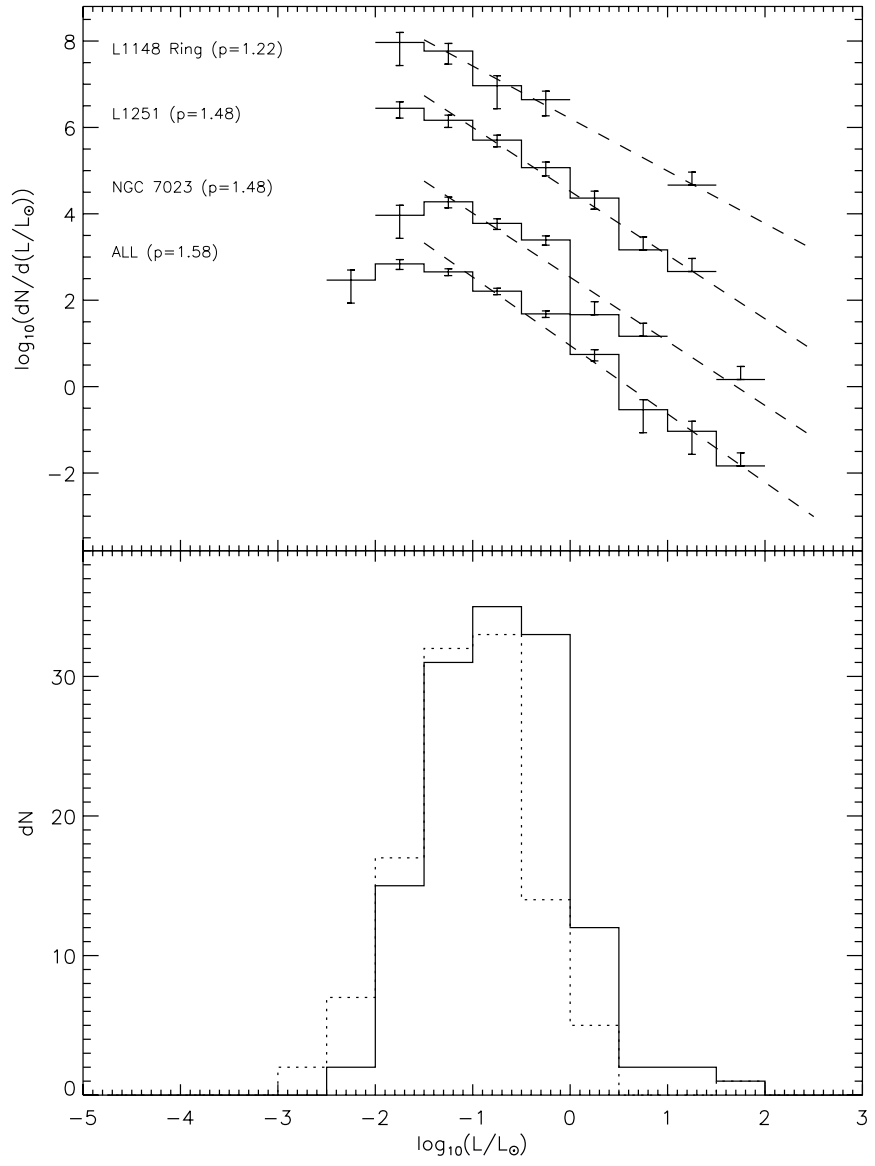

Figure 14. Top: bolometric luminosity functions for the 133 Spitzer-identified YSOs. Each luminosity function is offset by 2.0 dex from the preceding one. The index of the luminosity function is shown for each region. No lower limit is shown for bins with only one source. Lower: histogram of bolometric luminosity $\left(L_{\mathrm{bol}}\right)$ for all sources (solid line) and infrared luminosity $\left(L_{\mathrm{IR}}\right)$ for sources detected with IRAC (dashed line). Both plots show a break at our limiting luminosity of $L \sim 0.06 L_{\odot}$.

width and spectroscopic follow-up (Simon 2006; Kun \& Prusti 1993; Kun et al. 2008). The low number of transitional disk sources may also be indicative that most of the disks in this region are at an early evolutionary stage. By comparison, the Spitzer study of three clusters around Cep OB2 found that $10 \%$ of the detected disks were transitional in nature (Sicilia-Aguilar et al. 2006). Several sources have strong $70 \mu \mathrm{m}$ excesses, e.g., YSO \#16, 35, and 122. Similar objects are discussed in the Spitzer studies of Lupus (Merín et al. 2008) and Chamaeleon (Alcalá et al. 2008) and may represent a very young population of debris disks (Hillenbrand et al. 2008).

In Cepheus, there is a population of isolated $\mathrm{T}$ Tauri stars $\left(l \sim 117^{\circ}-122^{\circ}\right)$ that are unassociated with molecular material and are outside of the regions surveyed in this paper $\left(l<115^{\circ}\right)$. The isolation of these objects compared with their evolutionary status suggests that they formed in situ and that their natal clouds have been removed by an interaction with the Cepheus Flare Shell (Tachihara et al. 2005). If cloud disruption is as effective as argued by Tachihara et al. (2005) then it could explain why we are preferentially seeing young disks in our YSO sample. Our observations were specifically targeted toward dense clouds and would therefore be missing older YSOs whose clouds have already been dispersed. In the Appendix, a comparison of the YSO distribution to the pattern of extinction and $160 \mu \mathrm{m}$ emission shows that the L1228 South YSO group is on the very

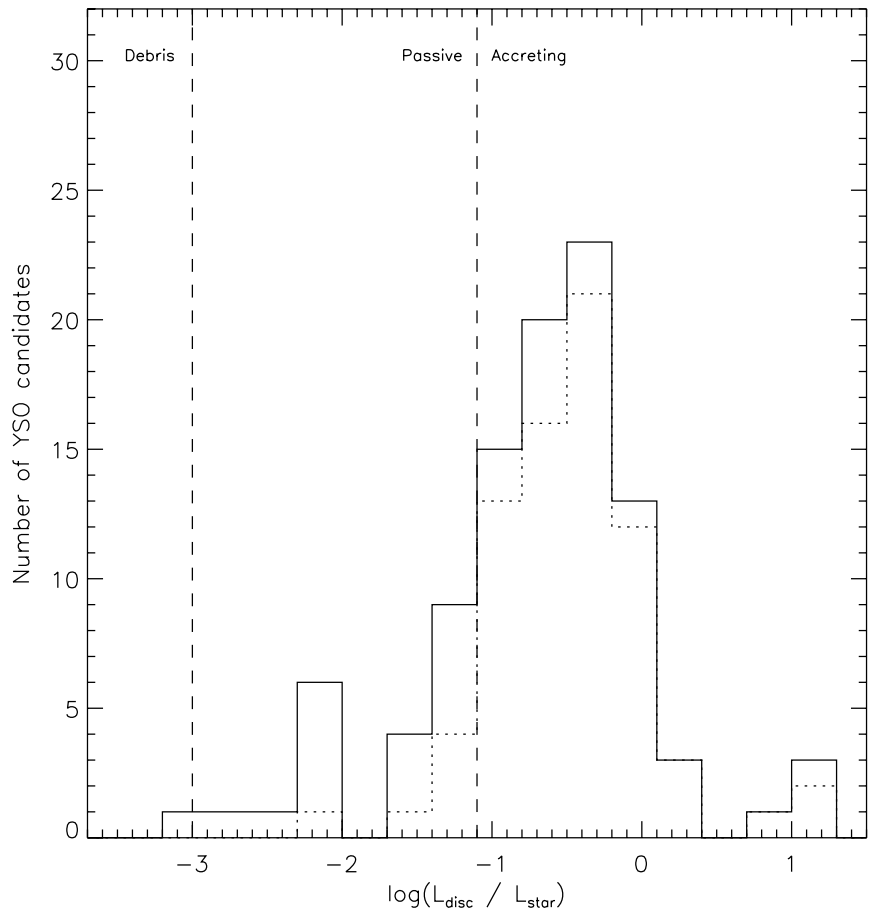

Figure 15. Histogram of the ratio of disk to star luminosity $\left(L_{\text {disk }} / L_{\text {star }}\right)$ as derived from the SED modeling for Class II and Class III candidate sources. The solid line is for all sources and the dashed line excludes sources identified with the 2MASS/MIPS scheme. The vertical dashed lines show the expected regions for accreting, passive, and debris disks. The histogram shows that the majority of the disks are of the accreting type.

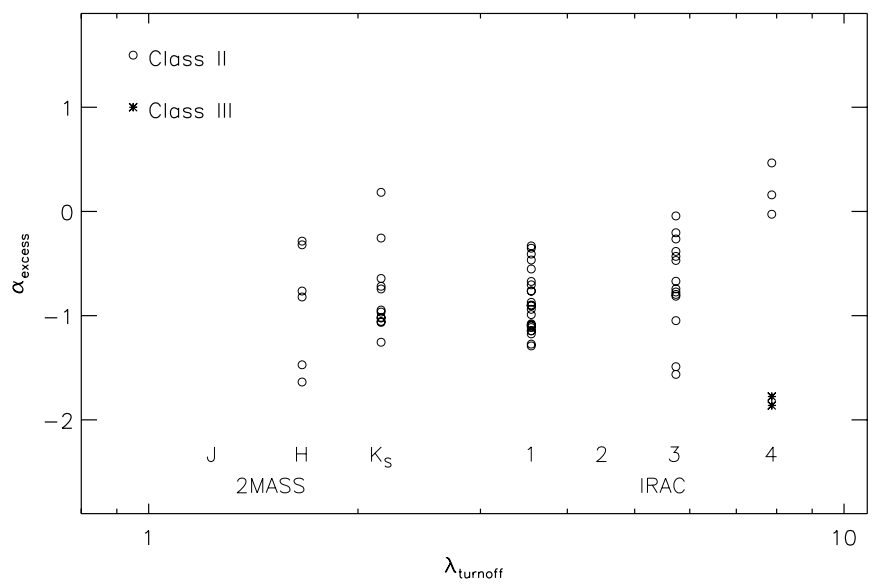

Figure 16. Plot of $\lambda_{\text {turnoff }}$, the wavelength where the dereddened SED diverges from the normalized stellar photosphere, against $\alpha_{\text {excess }}$ the spectral index of SED points longward of $\lambda_{\text {turnoff }}$. Each marker represents a single Class II or Class III candidate as shown by the key. The wavelength bands are labeled with their instrument band. The Class III candidates only show an excess at the longest values of $\lambda_{\text {turnoff }}$.

edge of the L1228 cloud. It is perhaps reasonable to suggest that the L1228 cloud is currently being disrupted and that we are witnessing the unveiling of a cluster of $\mathrm{T}$ Tauri stars similar to the isolated group observed by Tachihara.

\section{EXTENDED STRUCTURE AND YSO DISTRIBUTION}

Figure 17 shows the distribution of YSOs and visual extinction toward the regions in the Cepheus survey. Figure 18 shows the pattern of MIPS $160 \mu \mathrm{m}$ emission toward exactly the same regions. All regions are shown at the same angular scale. 

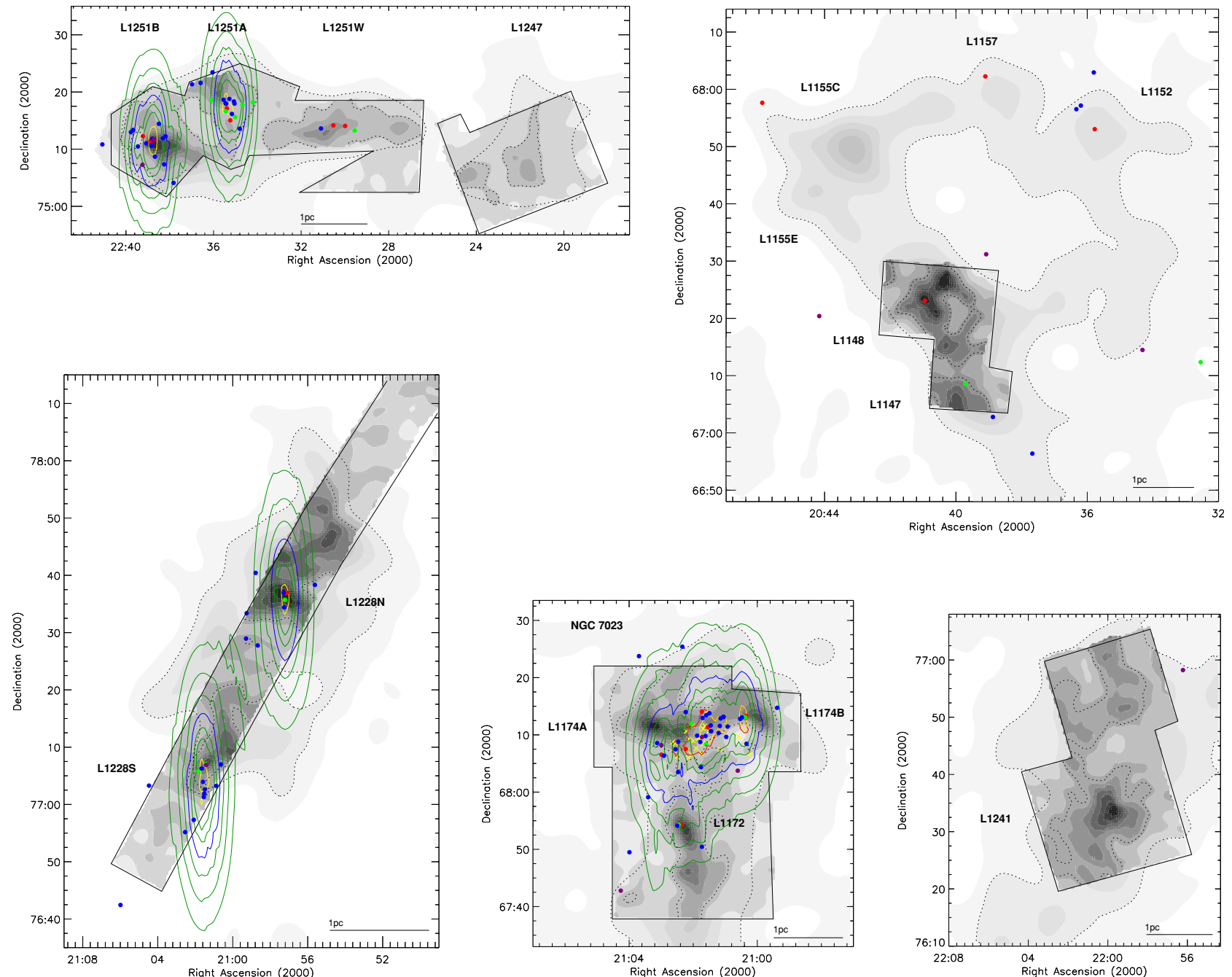

Figure 17. Distribution of YSO candidates, clustering results, and extended structure toward the Cepheus dark clouds associations. The gray scale shows the distribution of visual extinction derived via two different data sets. The extinction as derived from the Digitized Sky Survey (Dobashi et al. 2005) is shown across the entire map. The higher resolution Spitzer extinction maps are superimposed over this in the regions where there is IRAC/MIPS overlap (as shown by the boxes). Two dashed contours, Dobashi $A_{V}=1$ and Spitzer $A_{V}=5$, are shown. The colored markers show the location and spectral type of the YSO candidates. The colors are the same as used in Figure 9 (red/green/blue/purple for Class I/Flat/II/III). The colored contours, green unless noted, show $\rho_{*}$ from the clustering analysis in units of $0.125,0.25$, $0.5,1.0$ (blue), 2.0, 4.0, 25.0 (yellow) $\times M_{\odot} \mathrm{pc}^{-3}$.

(A color version of this figure is available in the online journal.)

\subsection{Comparison of $A_{V}$ and $160 \mu \mathrm{m}$ Maps}

Figure 17 shows $A_{\mathrm{V}}$ calculated from the Spitzer catalog. The line-of-sight extinction toward sources classified as reddened stellar photospheres between wavelengths of 2 and $24 \mu \mathrm{m}$ was calculated assuming an extinction law of $R=5.5$. These points were then filtered based on the equivalent 2MASS extinction to correct for contamination from isolated clumps of extinction. These irregularly spaced, filtered data were then averaged onto a regularly spaced grid using a Gaussian kernel (Evans et al. 2007; Huard et al. 2009). The resolution of the maps depends on the surface density of available sources. This density varies between regions in Cepheus and the highest resolution consistent between all regions was 150 arcsec.

The approximate areas where we have IRAC and MIPS overlap are shown by the irregular boxes in Figure 17. These are the regions where there is enough photometric coverage to calculate the Spitzer $A_{\mathrm{V}}$. The gray scale within the overlap regions shows the local range of the 2.5 arcmin resolution Spitzer $A_{\mathrm{V}}$ on a linear stretch. Outside of the overlap area, the gray scale shows the 6 arcmin resolution DSS extinction on a linear stretch between 0.5 and 3 mag (Dobashi et al. 2005). Dashed black contours outline the region where the DSS extinction is greater than $A_{\mathrm{V}}=1$ and the Spitzer extinction is greater than $A_{\mathrm{V}}=5$. The Spitzer extinction maps show higher values of $A_{\mathrm{V}}$ than the Dobashi maps because the infrared stars are visible at higher column densities.

Figure 18 shows the $160 \mu \mathrm{m}$ emission observed by MIPS toward the five regions in Figure 17. The gray scale is shown on a $\log$ stretch between the local minimum (approximately $20 \mathrm{MJy} \mathrm{sr}^{-1}$ ) and $316 \mathrm{MJy} \mathrm{sr}^{-1}$ for NGC 7023 or $100 \mathrm{MJy} \mathrm{sr}^{-1}$ for the rest. The $A_{\mathrm{V}}$ contours and the overlap box from Figure 17 are repeated for reference. There is a strong correlation between the distribution of the visual extinction and the $160 \mu \mathrm{m}$ emission. This pattern of the $160 \mu \mathrm{m}$ emission following the $A_{\mathrm{V}}$ map has also been seen in many other regions (e.g., Rebull et al. 2007; Chapman et al. 2007).

In general, the $160 \mu \mathrm{m}$ emission above $\sim 40 \mathrm{MJy} \mathrm{sr}^{-1}$ is confined within the $A_{\mathrm{V}}=1$ DSS contour. Some regions, particularly L1241 and L1247, also show a correlation at smaller 

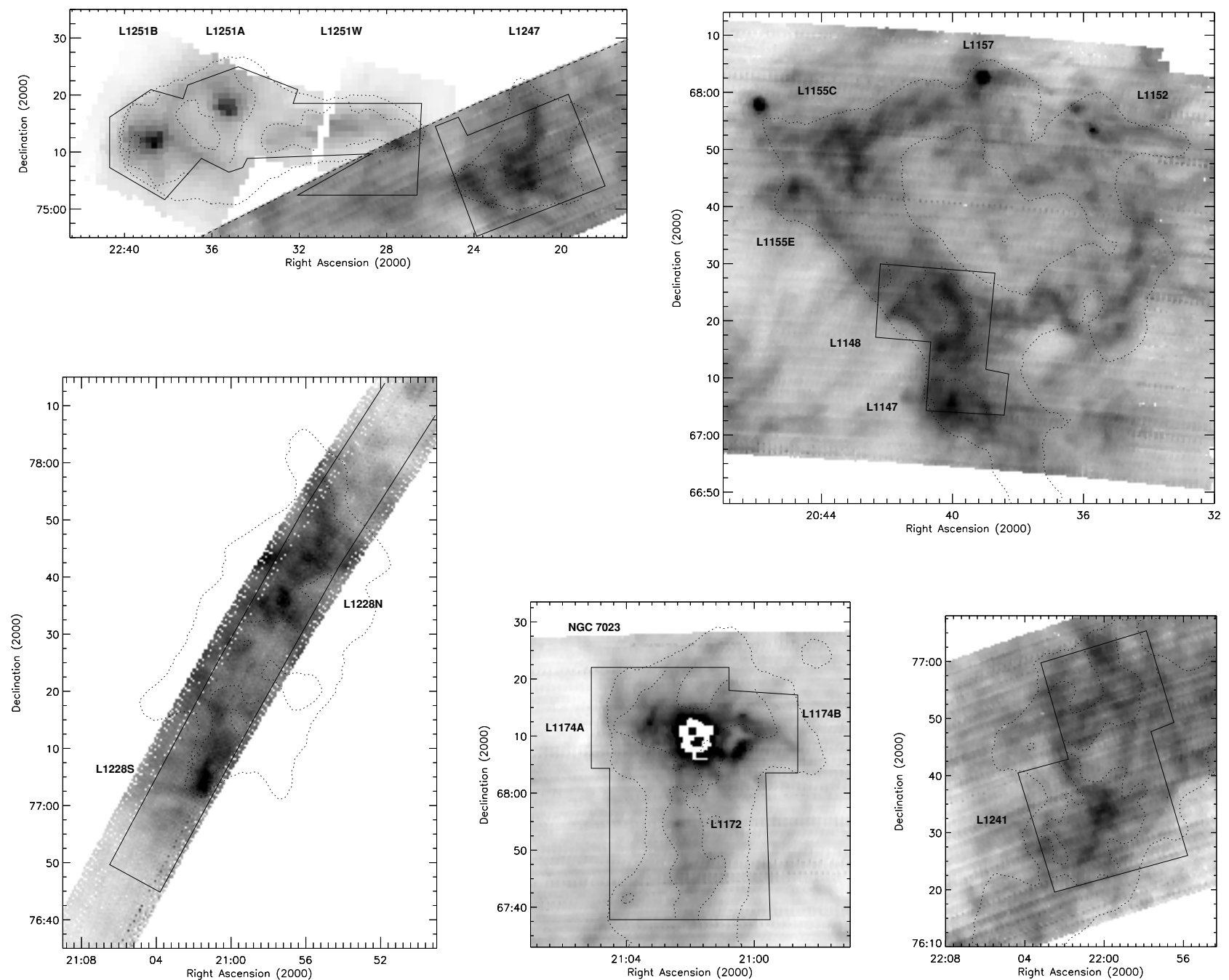

Figure 18. Distribution of MIPS $160 \mu \mathrm{m}$ emission toward the Cepheus dark cloud associations. The gray scale shows a log stretch between the local minimum ( $\sim 20 \mathrm{MJy} \mathrm{sr}^{-1}$ ) and $316 \mathrm{MJy} \mathrm{sr}^{-1}$ (NGC 7023) or $100 \mathrm{MJy} \mathrm{sr}^{-1}$ (all other regions). The Dobashi $A_{V}=1$ and Spitzer $A_{V}=5$ contours and the overlap boxes from Figure 17 are shown for reference. No MIPS $160 \mu \mathrm{m}$ data exist for L1251, so an ISOPHOT $200 \mu \mathrm{m}$ map scaled to the $160 \mu \mathrm{m}$ intensity in L1247 is shown instead (Lemke et al. 1996). The line separating the $200 \mu \mathrm{m}$ and $160 \mu \mathrm{m}$ maps is shown by a dashed line.

angular scales. There are also subtle differences, however. For example, in L1228S, the extinction peaks approximately 4 arcmin farther north than the $160 \mu \mathrm{m}$ emission. The center of NGC 7023 saturates the MIPS detectors and is left blank in the map.

Table 15 lists parameters for the clouds and YSO clusters/ groups shown in Figure 17. We identify dense cores based on a comparison of the extinction and $160 \mu \mathrm{m}$ maps. The $A_{\mathrm{V}}=5$ contour was chosen to define the cores as it was the level where most of them separated from each other. These are labeled in Figures 17 and 18. Due to the partial mapping of L1147 and the small size of L1155E, these regions are merged with L1148 and $\mathrm{L} 1155 \mathrm{C}$ when calculating derived properties. L1174A and L1174B, with the unlabeled L1174C, are listed together as NGC 7023. For each of the remaining 14 cores, we measured the peak and mean $A_{\mathrm{V}}$ within the $A_{\mathrm{V}}=5$ contour, the area contained within that contour, and the equivalent mass calculated from the $A_{\mathrm{V}} / N_{H}$ relationship of Bohlin et al. (1978). The c2d and Gould Belt $A_{\mathrm{V}}$ values are calculated assuming an extinction law of $R=5.5$. To use the Bohlin et al. (1978) relation of

$$
\frac{A_{\mathrm{V}}}{N_{\mathrm{H}_{2}}}=1.1 \times 10^{-21} \mathrm{~cm}^{-2} \mathrm{mag},
$$

which was calculated for a value of $R=3.1$, we include a correction of 0.716 . These values are listed in Columns 11-14 of Table 15. The equivalent values for the YSO clusters/groups are calculated using a YSO density contour that defines the edge of the group (see Section 6.3).

\subsection{YSO Distribution and Completeness}

The distribution of YSO candidates is overplotted in the five regions in Figure 17. The markers are color coded to the class of the candidate-red for Class 0/I, green for Flat spectrum, blue for Class II, and purple for Class III. Columns 2-6 of Table 15 list the number and type of YSO candidates that were detected toward each core within the $A_{\mathrm{V}}=1$ contour or the YSO group/ cluster boundary. Column 7 of Table 15 lists the ratio of Class I YSO (Column 2) to the total number of YSOs (Column 6). In general, the protostellar cores had such ratios in excess of $\sim 20 \%$, while the cores containing clusters had values less than $\sim 20 \%$.

A YSO was considered to be "off-cloud" if it was outside of the $A_{\mathrm{V}}=1$ contour and was not a member of a formal YSO cluster or group (see Section 6.3). The results for these YSO candidates are listed as a separate group at the bottom of 
Table 15

Statistics of Star-forming Dark Clouds in Cepheus

\begin{tabular}{|c|c|c|c|c|c|c|c|c|c|c|c|c|c|c|}
\hline \multirow[t]{2}{*}{ Dark Cloud } & \multicolumn{6}{|c|}{ Spitzer-identified YSOs ${ }^{\mathrm{a}}$} & \multicolumn{2}{|c|}{$\begin{array}{l}\text { non-S. } \\
\text { YSOs }^{b}\end{array}$} & \multirow{2}{*}{$\begin{array}{c}N_{\mathrm{YSO}} \\
A_{V}>5\end{array}$} & \multicolumn{2}{|c|}{$A_{V}$} & \multirow{2}{*}{$\begin{array}{l}\text { Area }^{\mathrm{c}} \\
\left(\mathrm{pc}^{-2}\right)\end{array}$} & \multirow{2}{*}{$\begin{array}{c}M_{\text {cloud }} \\
\left(M_{\odot}\right)\end{array}$} & \multirow{2}{*}{$\begin{array}{l}\text { SFE } \\
(\%) \\
\end{array}$} \\
\hline & I & $\mathrm{F}$ & II & III & Tot. & $\begin{array}{c}\text { I/Tot. } \\
(\%)\end{array}$ & Inc. & Tot. & & $\begin{array}{c}\text { Max } \\
(\operatorname{mag})\end{array}$ & $\begin{array}{l}\text { Mean } \\
(\mathrm{mag})\end{array}$ & & & \\
\hline \multicolumn{15}{|c|}{ Prestellar Cores } \\
\hline L1155 & 0 & 0 & 0 & 0 & 0 & 0 & 0 & 0 & 0 & $\ldots$ & $\ldots$ & $\ldots$ & $\ldots$ & $\ldots$ \\
\hline L1241 & 0 & 0 & 0 & 1 & 1 & 0 & 0 & 0 & 0 & 11.2 & 6.2 & 0.058 & 150 & 0 \\
\hline L1247 & 0 & 0 & 0 & 0 & 0 & 0 & 0 & 0 & 0 & 8.9 & 6.2 & 0.015 & 38 & 0 \\
\hline Sub Total & 0 & 0 & 0 & 1 & 1 & & 0 & 0 & 0 & & & 0.073 & 188 & \\
\hline \multicolumn{15}{|c|}{ Protostellar Cores (peak $\rho_{*}<$ disruption density) } \\
\hline L1148+L1147 & 1 & 1 & 2 & 0 & 4 & 25 & 0 & 0 & 2 & 8.8 & 6.0 & 0.051 & 150 & 1 \\
\hline L1152 & 1 & 0 & 2 & 0 & 3 & 33 & 0 & 0 & 0 & $\ldots$ & $\ldots$ & $\ldots$ & $\ldots$ & $\ldots$ \\
\hline L1157 & 0 & 0 & 0 & 0 & 0 & 0 & 1 & 1 & 0 & $\ldots$ & $\ldots$ & $\ldots$ & $\ldots$ & $\ldots$ \\
\hline L1172 & 1 & 1 & 3 & 1 & 6 & 17 & 0 & 0 & 4 & 13.1 & 7.0 & 0.040 & 107 & 2 \\
\hline L1221 & 3 & 0 & 0 & 0 & 3 & 100 & 0 & 0 & 0 & $\ldots$ & $\ldots$ & $\ldots$ & $\ldots$ & $\ldots$ \\
\hline L1251W & 2 & 1 & 1 & 0 & 4 & 50 & 0 & 0 & 4 & 11.7 & 6.7 & 0.040 & 110 & 2 \\
\hline Sub Total & 8 & 3 & 8 & 1 & 20 & & 1 & 1 & 10 & & & 0.131 & 367 & 1 \\
\hline \multicolumn{15}{|c|}{ Cores with YSO Groups/Clusters (peak $\rho_{*}>$ disruption density) } \\
\hline NGC 7023 Core & 6 & 3 & 26 & 2 & 37 & 16 & 4 & 6 & 34 & 14.9 & 7.0 & 0.040 & 107 & 14 \\
\hline ... Loose & 5 & 3 & 23 & 1 & 32 & 16 & 4 & 6 & & & 5.7 & 0.078 & 169 & 9 \\
\hline ... Tight A & 1 & 2 & 7 & 0 & 10 & 10 & 3 & 3 & & 8.5 & 6.0 & 0.004 & 8 & 38 \\
\hline ... Tight B & 2 & 1 & 2 & 0 & 5 & 40 & 0 & 0 & & 10.7 & 6.3 & 0.001 & 2 & 57 \\
\hline L1228N Core & 2 & 1 & 7 & 0 & 10 & 20 & 0 & 0 & 6 & 12.9 & 7.7 & 0.059 & 84 & 3 \\
\hline ... Loose & 2 & 1 & 2 & 0 & 5 & 40 & 0 & 0 & & & 7.0 & 0.021 & 27 & 9 \\
\hline . . . Tight & 2 & 2 & 2 & 0 & 6 & 33 & 0 & 0 & & & 9.1 & 0.001 & 2 & 65 \\
\hline L1228S Core & 0 & 2 & 9 & 0 & 11 & 0 & 2 & 3 & 8 & 10.0 & 6.5 & 0.028 & 34 & 10 \\
\hline ... Loose & 4 & 2 & 7 & 0 & 13 & 31 & 2 & 3 & & & 4.7 & 0.016 & 14 & 31 \\
\hline ... Tight & 4 & 0 & 2 & 0 & 6 & 67 & 0 & 1 & & & 6.2 & 0.001 & 1 & 69 \\
\hline L1251A Core & 1 & 4 & 11 & 0 & 16 & 6 & 2 & 2 & 17 & 14.6 & 7.3 & 0.026 & 79 & 10 \\
\hline ... Loose & 1 & 3 & 8 & 0 & 12 & 8 & 1 & 1 & & & 6.4 & 0.016 & 42 & 12 \\
\hline ... Tight & 0 & 1 & 2 & 0 & 3 & 0 & 1 & 1 & & & 6.9 & 0.001 & 3 & 32 \\
\hline L1251B Core & 4 & 0 & 12 & 3 & 19 & 21 & 0 & 1 & 17 & 22.1 & 8.3 & 0.042 & 144 & 6 \\
\hline$\ldots$ Loose & 4 & 0 & 9 & 3 & 16 & 25 & 0 & 0 & & & 7.6 & 0.022 & 68 & 11 \\
\hline$\ldots$ Tight & 3 & 0 & 2 & 2 & 7 & 43 & 0 & 0 & & & 14.3 & 0.002 & 13 & 22 \\
\hline Sub Total Core & 13 & 10 & 65 & 5 & 93 & 14 & 8 & 12 & 82 & $\ldots$ & $\ldots$ & 0.195 & 447 & 8 \\
\hline$\ldots$ Loose & 16 & 9 & 49 & 4 & 78 & 21 & 7 & 10 & & $\cdots$ & $\ldots$ & 0.153 & 320 & 11 \\
\hline $\begin{array}{l}\ldots \text { Tight } \\
\text { Off Cloud }\end{array}$ & 12 & 6 & 17 & 2 & 37 & 32 & 4 & 5 & & $\ldots$ & $\ldots$ & 0.010 & 29 & 39 \\
\hline Sub Total & 0 & 1 & 14 & 4 & 19 & 0 & 1 & 2 & $\ldots$ & $\ldots$ & $\ldots$ & $\ldots$ & $\ldots$ & $\ldots$ \\
\hline Grand Total & 21 & 14 & 87 & 11 & 133 & 16 & 10 & 15 & 92 & $\ldots$ & $\ldots$ & 0.400 & 1003 & 4 \\
\hline
\end{tabular}

Notes.

a The number of Spitzer-identified YSO candidates by Class (as determined by the $\alpha_{\mathrm{IR}}$ spectral index) associated with the named core (within the projected $A_{V}=1$ contour) or cluster (within the relevant tight or loose contour).

b The number of non-Spitzer-identified YSOs as listed in Table 16. The column labeled "Inc." lists the number of non-Spitzer-identified YSOs that were coincident with entries in the Cepheus catalog. The column labeled "Tot." lists the total number of YSOs missed by the Spitzer YSO schemes in that region.

c The area of the region used to calculate the mean $A_{V}$, the cloud mass $M_{\text {cloud }}$, and the star-formation efficiency (SFE). For a core, this is the area that is enclosed by the $A_{V}=5$ contour. For a loose and tight cluster, this is the area that is enclosed by the $\rho_{*}=1.0$ and $25 M_{\odot}$ pc ${ }^{-3}$ contours, respectively.

Table 15. The isolated population of Weak-Line T Tauri YSOs in Cepheus have higher YSO-to-cloud separation $(\sim 10 \mathrm{pc})$ than that found in other star-formation regions (Tachihara et al. 2005). While our observations do not get that far "off-cloud," we can use the number of off-cloud YSOs, most of which are the Class II and Class III sources, to estimate the density of off-cloud YSOs that are serendipitously located close to dense regions. The area at an extinction of $A_{\mathrm{V}}>1$ is $2.14 \mathrm{deg}^{2}$, and the total mapped area is $8.33 \mathrm{deg}^{2}$. We detect 19 Spitzeridentified YSOs at an extinction of $A_{\mathrm{V}}<1$. Assuming that these are not background galaxies, we estimate an off-cloud YSO density of $19 /(8.33-2.14)=3.1 \mathrm{YSOs} \mathrm{deg}^{-2}$. Conversely, we detect 133-19 = 114 Spitzer -identified YSOs at an extinction of $A_{\mathrm{V}}>1$. This gives an on-cloud YSO surface density of $114 / 2.14=53$ YSOs $\operatorname{~eg}^{-2}$.

The SIMBAD object types (the "otype" field) of the sources listed in the first three sections of Table 6 include YSOs and YSO candidates $\left(\mathrm{Y}^{*} 0\right.$ and $\mathrm{Y}^{*}$ ?), emission-line stars $\left(\mathrm{EM}^{*}\right)$, variable star of various classes $\left(\mathrm{V}^{*}, \mathrm{Pu}^{*}, \mathrm{Mi}^{*}, \mathrm{Or} *\right)$, reflection nebulae (RNe), and T Tauri stars (TT*). A number of sources have multiple object types. A search was made for additional sources with similar object types in a region coincident with our mapped area. The result included 14 variable stars located around NGC 7023 and toward the L1148+L1155 ring. Only 
Table 16

Nonidentified SIMBAD YSOs

\begin{tabular}{|c|c|c|c|c|c|c|}
\hline Source Name ${ }^{a}$ & $\begin{array}{c}\text { R.A. } \\
(2000)\end{array}$ & $\begin{array}{c}\text { Decl. } \\
(2000)\end{array}$ & Origin $^{b}$ & Region & $\begin{array}{c}\text { Spitzer } \\
\text { Catalog. }^{\mathrm{c}}\end{array}$ & $\begin{array}{c}\text { Spitzer } \\
\text { YSO Id }^{\mathrm{d}}\end{array}$ \\
\hline L1157 & 20:39:06.2 & $+68: 02: 15$ & $\mathrm{~S}$ & L1157 & $\mathrm{Y}$ & 134 \\
\hline PV Cep & $20: 45: 53.9$ & $+67: 57: 39$ & $\mathrm{~S}$ & Off-cloud & $\mathrm{Y}$ & 135 \\
\hline IRAS F20597+6800 & $21: 00: 18.4$ & $+68: 12: 02$ & $\mathrm{~S}$ & NGC 7023 & $\mathrm{~N}$ & $\ldots$ \\
\hline L1228 S1(b) & 21:01:25.1 & $+77: 06: 54$ & $\mathrm{~K}$ & L1228 & $\mathrm{Y}$ & 136 \\
\hline NGC 7023 RS 2 & 21:01:27.1 & $+68: 10: 38$ & $\mathrm{~K}$ & NGC 7023 & $\mathrm{Y}$ & 137 \\
\hline IRAS F21022+7651 & $21: 01: 29.4$ & $+77: 03: 45$ & $\mathrm{~S}$ & L1228 & $\mathrm{N}$ & $\ldots$ \\
\hline HD 200775 & $21: 01: 36.9$ & $+68: 09: 48$ & $\mathrm{~S}$ & NGC 7023 & $\mathrm{Y}$ & 138 \\
\hline NGC 7023 RS 3 & $21: 01: 43.6$ & $+68: 09: 36$ & $\mathrm{~K}$ & NGC 7023 & $\mathrm{Y}$ & 139 \\
\hline IRAS F21025+7645 & 21:01:49.2 & $+76: 57: 51$ & $\mathrm{~S}$ & L1228 & $\mathrm{Y}$ & 140 \\
\hline NGC $7023 \mathrm{~S}$ T & 21:02:07.9 & $+68: 09: 16$ & $\mathrm{~S}$ & NGC 7023 & $\mathrm{~N}$ & $\ldots$ \\
\hline IRAS F21025+6801 & $21: 03: 12.0$ & $+68: 13: 02$ & $\mathrm{~S}$ & NGC 7023 & $\mathrm{Y}$ & 141 \\
\hline GSC 04600-02450 & $22: 34: 11.9$ & $+75: 10: 10$ & $\mathrm{~S}, \mathrm{~K}$ & L1251A & $\mathrm{Y}$ & 142 \\
\hline TW96 N1a & $22: 35: 23.3$ & $+75: 17: 09$ & $\mathrm{~S}$ & L1251A & $\mathrm{Y}$ & 143 \\
\hline XMMU J223727.7+751525 & $22: 37: 27.5$ & $+75: 15: 25$ & $\mathrm{~S}, \mathrm{~K}$ & $\mathrm{~L} 1251 \mathrm{~B}$ & $\mathrm{~N}$ & $\ldots$ \\
\hline KP93 3-19 & $22: 40: 58.5$ & $+75: 03: 56$ & $\mathrm{~S}$ & L1251 (Off-cloud) & $\mathrm{N}$ & $\ldots$ \\
\hline
\end{tabular}

Notes.

a Name of the source in either the SIMBAD database or Kun et al. (2008).

$\mathrm{b}$ The origin of the YSO candidate. S, a YSO or pre-main-sequence listing in the SIMBAD database; K, a T Tauri star from Kun et al. (2008).

${ }^{c}$ Does the source have an entry in the Cepheus Spitzer catalog? In this instance, the only condition for inclusion is to have a line entry in the Spitzer catalog irrespective of whether that source was formally detected in any particular band.

d The YSO identification number from Table 6 for sources that are found in the Spitzer Cepheus catalog.

HD 200775, however, had a match against a source in the Spitzer catalog. Kun et al. (2008) reviewed surveys of $\mathrm{H} \alpha$ emission line stars toward the Cepheus Flare and produced a list of emission line stars that had been confirmed spectroscopically as T Tauri stars. There are 19 emission-line stars in the list of additional SIMBAD sources. The emission-line stars were preferentially found toward L1228 as this area was the subject of an $\mathrm{H} \alpha$ survey by Ogura \& Sato (1990). Thirty-one of the Kun et al. (2008) T Tauri stars are coincident with Spitzer YSO candidates, but none of the additional SIMBAD emission line stars are confirmed as a T Tauri star.

The SIMBAD search was narrowed to only include objects previously identified as YSOs and pre-main-sequence stars or candidates for either. A total of 47 YSOs were found in the SIMBAD database of which 36 were cross-identified with the Spitzer YSO candidates. Half of these cross-identified YSOs are in the L1251B region and are from the Lee et al. (2006) c2d paper. Excluding these, 18 out of the 30 SIMBAD YSOs were cross-identified with the Spitzer YSO candidates. The 12 SIMBAD YSOs that were not identified by the Spitzer schemes plus three T Tauri stars from Kun et al. (2008) that were not identified by the Spitzer schemes are listed in Table 16. Column 4 lists the catalog that the object was taken from (S for SIMBAD, $\mathrm{K}$ for Kun et al. 2008). Column 5 lists the core or the region coincident with the object's position. Column 6 lists whether the object position was coincident with an entry in the Cepheus Spitzer catalog even if that object was not classified as a YSO candidate or formally detected. Ten of the additional YSOs were coincident with entries in the SGBS Cepheus catalog (PV Cep had only 2MASS data). Each of these non-Spitzer-identified YSO candidates has been assigned a YSO Id (as listed in Column 7) and their photometry is listed in the fourth section of Table 6.

The embedded Class 0 protostar L1157 was outside of our IRAC area and has no 2MASS detection. L1157 was studied with Spitzer by Looney et al. (2007). They presented striking $8 \mu \mathrm{m}$ images of L1157 that showed its CO outflow in emission and a flattened circumstellar envelope in absorption against the bright $8 \mu \mathrm{m}$ background. Its IRAC fluxes have been added to our catalog based on 4.5 arcsec radius aperture photometry conducted on post-BCD data downloaded from the Spitzer data archive. Spitzer data for PV Cep exist in the data archive, ${ }^{15}$ but these are saturated at all four IRAC wavelengths. The 2MASS/ MIPS YSO \#118 is also in the PV Cep IRAC field so we add its IRAC fluxes to the catalog in the same manner as for L1157.

HD 200775 would have been picked up by the IRAC YSO scheme if we had reduced the signal-to-noise ratio $(\mathrm{S} / \mathrm{N})$ cut to 2. It was detected in the filters used for the 5-Band scheme, but it was excluded because the $3.6 \mu \mathrm{m}$ flux was below $2 \sigma$. The source is very luminous $\left(L_{\mathrm{bol}}=100 L_{\odot}\right)$ and is saturated in all MIPS bands. It is illuminating the surrounding gas, giving it a larger flux uncertainty than an isolated star would have. The position of NGC $7023 \mathrm{~S}$ T (Sellgren 1983) was identified with IRAS F21023+6754 by Kun (1998). Kun also identified the sources IRAS F21025+6801 and F20597+6800 as IRAS YSO candidates within the NGC 7023 region. Of these latter three sources, only IRAS F21025+6801 is coincident with an entry in the Cepheus catalog and then it only has a $24 \mu \mathrm{m}$ upper limit. None of the Tachihara et al. (2005) $\mathrm{x}$-ray $\mathrm{T}$ Tauri candidates were coincident with the area we surveyed.

A total of 143 YSOs were identified from the Spitzer catalog and the SIMBAD database. Of these, 133 (93\%) were identified by the color-color schemes discussed in Section 4.1, and 10 (7\%) were coincident with entries in the catalog. The number of previously known YSOs that were not identified by Spitzer, but had entries in the Cepheus catalog, is listed in Column 8 of Table 15. The total number of known YSOs that were not identified by Spitzer is listed in Column 9 of Table 15. The number of Spitzer-identified YSO candidates within $A_{\mathrm{V}}=$ 5 contours discussed in the previous section is listed in Column 10 of Table 15.

\footnotetext{
15 AOR No. 18955008
} 


\subsection{Prestellar Cores, Protostellar Cores, and Cores with YSO Groups/Clusters}

The dense cores seen in extinction in Cepheus can be split into three groups. The first division is prestellar cores. These are cores that are believed to be taking part in the star-formation process but have not yet formed an embedded YSO (i.e., $N_{\text {YSO }}=0$ ). We then define the cores that have $N_{\text {YSO }}>0$ within the $A_{\mathrm{V}}=5$ contour as protostellar cores. Concentrations of YSOs in protostellar cores with at least 5 and 35 members are termed groups and clusters respectively (Lada \& Lada 2003).

For prestellar cores, the YSO count within the $A_{\mathrm{V}}=5$ contour is used because the area subtended by the $A_{\mathrm{V}}=1$ contour can be large enough to give a significant chance of including a field YSO. For example, the area of L1241 at $A_{\mathrm{V}}>1$ is approximately $0.6 \mathrm{deg}^{2}$. Based on the average off-cloud YSO surface density, we would expect 1-2 YSOs to be coincident with this region. There is one Class III YSO just inside the $A_{\mathrm{V}}=1$ border of L1241. Given its class and the likelihood of finding a coincident YSO within that border, we conclude that L1241 is starless. The parameters for the Cepheus prestellar cores are listed in the first section of Table 15 . The respective masses and areas are calculated from the $A_{\mathrm{V}}=5$ contour.

The cores that have $N_{\text {YSO }}>0$ are termed protostellar cores. The distribution of YSOs in Figure 17 shows a number of protostellar cores. There have been multiple metrics devised for classifying the composition and concentration of YSOs in protostellar cores. For example, Cartwright \& Whitworth (2004) used minimal spanning trees to derive a quantity $Q$ that could differentiate between a small, large-scale density gradient and multiscale subclustering. Alternatively, the spatial density of sources can be estimated by calculating the angular distance $r_{\mathrm{N}}$ to the $N$ th nearest neighbor for each point on a regularly spaced grid (Christopher et al. 1998; Gladwin et al. 1999; Gutermuth et al. 2005).

For consistency with previous c2d/SGBS papers, we follow the nearest-neighbor clustering scheme described by Gutermuth et al. (2005). When calculating $r_{\mathrm{N}}$, the appropriate correction for spherical coordinates was used (this is vital for Cepheus due to its high declination). This angular distance is then used to calculate a volume number density assuming a spherically symmetric distribution of cluster members (Gutermuth et al. 2005). Following Gutermuth et al. (2005), the volume density of stars is given by

$$
\rho_{*}=M_{*} \frac{N-1}{\frac{4}{3} \pi r_{\mathrm{N}}^{3}},
$$

where $M_{*}$ is the average stellar mass and $N$ is the chosen index for the neighbor. We use $N=5$ and assume an IMF with an average stellar mass of $0.5 M_{\odot}$ for consistency with other c2d and SGBS papers (e.g., Jørgensen et al. 2008; Harvey et al. 2008). The value of $\rho_{*}$ was calculated across the regions in our survey. A limitation to this technique is that it can only calculate $\rho_{*}$ for groups with five or more members.

The value of $\rho_{*}$ above which a cluster is stable against disruption from passing interstellar clouds is $\rho_{*} \geqslant 1.0 M_{\odot} \mathrm{pc}^{-3}$ (Spitzer 1958; Lada \& Lada 2003). Cores with peak values of $\rho_{*}$ less than the disruption density or with fewer than $N$ members are listed as purely protostellar and are listed in the middle section of Table 15.

The cores with peak values of $\rho_{*}$ greater than the disruption density are listed in the third section of Table 15 . Five regions had stellar mass volume densities in excess of the $1.0 M_{\odot} \mathrm{pc}^{-3}$ disruption density. These are NGC 7023/L1172, L1251A, L1251B, L1228S, and L1228N. For this analysis, we have only included the YSO candidates toward NGC 7023 and not the main-sequence members listed in the literature. The pattern of $\rho_{*}$ toward each of these groups is shown by the colored contours in the upper panels of Figure 17. The blue contour traces the disruption density, while green contours trace values of 0.125 , $0.25,0.5,2.0$, and 4.0 times the disruption density.

Figure 17 shows that the disruption contours are approximately elliptical, likely because these relatively small and isolated YSO groups are themselves elongated. Jørgensen et al. (2008) defined an empirical limit of 25 times the disruption density at a level that appears to more adequately trace the local YSO distribution. This level is shown as a yellow contour in Figure 17. The red contour over NGC 7023 shows the equivalent of the yellow contour after known non-YSO cluster members are included. The position of the 21 non-YSO cluster members is shown by yellow crosses.

Each group can be classified by the number of members within a particular contour. YSOs within a minimum contour equal to the disruption contour (blue contour) are described as a "loose" association and YSOs within a minimum contour equal to 25 times the disruption density (yellow contour) are described as a "tight" association (Jørgensen et al. 2008). A cluster is defined as an association with at least 35 members (Lada \& Lada 2003). Based on these definitions, no association in Cepheus can be considered a true cluster although NGC 7023 is close. It is a loose association of 32 members that contains two tight groups, A and B, with 20 and 4 members each. The other four cores contain individual YSO groups that have tight and loose members, although the tight regions do not fragment as in NGC 7023.

We have defined four different contours that can be used to define the extent of the cores. These are the $A_{\mathrm{V}}=1$ and $A_{\mathrm{V}}=5$ contours used for the cores themselves and the $\rho_{*}=1$ and $25 M_{\odot} \mathrm{pc}^{-3}$ used for the YSO groups/clusters. The third section of Table 15 lists the YSO statistics and physical parameters for the cores with YSO groups. Each core is listed three times, first for the core itself as defined by the $A_{\mathrm{V}}=1$ contour, then for the loose and tight YSO groups as defined by the blue and green contours in Figure 17. The relevant contour is used for the calculation of the group/cluster statistics. The tight groups for NGC 7023 A and B are listed separately.

In general, the statistics for the entire core and the loose groups are approximately equal, but there are a few noticeable differences. The statistics calculated from the "loose" contour characterize the cores with groups better than the $A_{\mathrm{V}}=5$ contour. The NGC 7023 loose cluster subtends an area that is significantly larger than the area of the core as measured by its $A_{\mathrm{V}}=5$ contour. The L1228 YSO group straddles both $A_{\mathrm{V}}$ contours, so using those contours to count the YSOs gives an artificially low number.

The final column in Table 15 lists the star-formation efficiency (SFE) for each core as given by

$$
\mathrm{SFE}=\frac{M_{\text {stars }}}{M_{\text {cloud }}+M_{\text {stars }}},
$$

where $M_{\text {cloud }}$ is the mass of the gas and dust in the core (as listed in Column 14 of Table 15) calculated from the mean $A_{\mathrm{V}}$ within the core's boundary contour. The mass of the stars, $M_{\text {stars }}$, is given by $N_{\mathrm{YSO}} M_{*}$ where $M_{*}$ is the same average stellar mass as assumed for the calculation of $\rho_{*}$. 
There is no clear trend between the masses of the cores and the number of YSOs they contain. Thus, a larger stellar content automatically translates into a higher star-formation efficiency. The protostellar cores without YSO groups/clusters have SFEs $\sim 1 \%$ while the overall SFE for the cores with YSO groups/clusters is $\sim 8 \%$. The SFE for the tight component of the groups/clusters is $>3 \times$ higher than for the loose component. This can be interpreted as either an increase in the in situ star formation within the dense parts of the core or a drift of YSOs into the central part of the core from other parts of the cloud. In the former, the SFE is real and, in the latter, it is overestimated because the mass of gas that those stars formed from is underestimated.

The bottom line of Table 15 shows the aggregated statistics for the entire mapped region. Columns 2-5 reiterate the YSO class statistics from Column 2 of Table 8. Table 17 lists additional star-formation statistics in the same format as Table 3 of Evans et al. (2009). Columns 1-4 of Table 17 list the boundary used for the analysis, the number of YSOs within that boundary $N_{\text {YSO }}$, the solid angle of the region $\Omega$, and the integrated area of the region $A$ assuming an average distance of $300 \mathrm{pc}$. Columns 5 and 6 respectively list the number of YSOs per square degree and square parsec. Column 7 lists the star-formation rate (SFR $\left.=N_{\mathrm{YSO}} M_{*} / \tau_{\mathrm{sf}}\right)$, where we have used the aforementioned assumption that $M_{*}=0.5 M_{\odot}$ (the same used in the clustering analysis) and that the timescale for star formation is $\tau_{\mathrm{sf}}=$ 2 Myr (Evans et al. 2009). Column 8 lists the star-formation rate per square parsec (SFR/A). Columns 9 and 10 list the total mass of the cloud $\left(M_{\text {cloud }}\right)$ and the surface density of the cloud ( $\left.M_{\text {cloud }} / A\right)$.

\section{DISCUSSION: STAR FORMATION IN THE CEPHEUS FLARE}

Star formation in Cepheus is mostly concentrated in small, isolated groups of YSOs. A total of 78 out of the 133 Spitzeridentified YSOs, i.e., 59\%, were determined to be members of loose YSO groups, leaving $41 \%$ of the YSOs distributed elsewhere. By comparison, the average distributed YSO fraction in c 2 d clouds was only $\sim 10 \%$ (Evans et al. 2009). Figure 19 shows the distribution of YSO and YSO candidates toward the Cepheus Flare plotted over the distribution of $\mathrm{H} \alpha$ (log scaled gray scale; Finkbeiner 2003) and integrated CO emission (black contours; the same data as the white contours from Figure 1, Dame et al. 2001). The black footprints show the limit of our survey, and the red crosses show the positions of Spitzer YSO candidates from this paper. The positions of spectroscopically confirmed $\mathrm{T}$ Tauri stars are shown by open blue squares (Kun et al. 2008) and the position of X-ray WTTS is shown by filled blue squares (Tachihara et al. 2005). The green circles show an unvetted list of objects described in the SIMBAD database as YSOs irrespective of their survey of origin.

The masses of cores with YSO groups $\left(N_{\mathrm{YSO}} \geqslant 5\right)$ and cores with only a few YSOs $\left(N_{Y S O}<5\right)$ were found to be broadly similar, which suggests that a factor other than core mass determines how many stars form. If we examine the location of the YSO groups (L1251A, L1251B, L1228S, and L1228N) in Figure 19, we see that they are all located in the region nearest the Cepheus Flare Shell (CFS), whereas the majority of the cores with less than five YSOs (L1148, L1157, etc.) are located on the opposite site of the region. There appears to be two different modes of star formation occurring here. The first mode of groups of YSOs has an SFE of $\sim 8 \%$ and has produced a YSO population that is dominated by Class II sources. The second unperturbed, almost quiescent mode has an SFE of $\sim 1 \%$ and has produced YSOs with a fairly uniform spread of infrared classes.

There is mounting evidence that star formation in the Cepheus Flare is triggered/influenced by the passage of the CFS (Grenier et al. 1989; Tachihara et al. 2005; Kun et al. 2008). Kun (1998) noted that the surface distribution of IRAS-identified YSOs in the Cepheus Flare peaked toward the edges of clouds and inferred that their formation was triggered by external shocks. Likewise, Figure 19 shows that the locations of the small YSO groups in L1228 and L1251 are on the edge of the CO contours facing the Cepheus Void. The cometary shape of $\mathrm{C}^{18} \mathrm{O}$ emission in L1251 and an unusual velocity gradient across the cloud appear to have been caused by interaction with a passing wind/shock wave, but the failure to detect $\mathrm{SiO}$ emission from the cloud may indicate that the shock phase, at least in L1251, has passed (Sato et al. 1994). It is the cometary head of L1251 that contains the L1251A and B YSO groups.

The L1228S YSO group not only lies on the edge of the CO contours but, as shown in Figure 17, it also lies on the edge of the DSS $A_{\mathrm{V}}=1$ and Spitzer $A_{\mathrm{V}}=5$ extinction contours. The peak of the L1228S Spitzer $A_{\mathrm{V}}$ map is offset from the peak of the $160 \mu \mathrm{m}$ emission. The difference in position could be due to heating of the dust caused as the core is dispersed by interaction with the CFS. There are small collections of T Tauri stars (open blue boxes) on the Void side of both the L1228 and L1251 groups, suggesting that they have become exposed as material has been stripped away from the leading edge of the molecular clouds. For the group of T Tauri stars near L1251, Tóth et al. (1995) estimated that it would have taken 0.1 Myr after the passage of the shock for an offset of $1^{\circ}$ to form, assuming an expansion velocity of $10 \mathrm{~km} \mathrm{~s}^{-1}$.

There is a collection of SIMBAD YSOs and T Tauri stars close to the end of the $\mathrm{CO}$ filament at $l=112^{\circ}, b=+14^{\circ}$. It is outside of our selection criteria (it has peak $A_{\mathrm{V}}<3$ ), but it is coincident with the L1235 dark nebula. Given the position of the YSOs, the CO contour, and its orientation to the Cepheus Void, it is possible that L1235 is another small YSO group, but one that is intermediate in evolution between L1228S and the Cepheus Void WTTS Group. Two other concentrations of YSOs are shown in Figure 19: L1199 at a distance of 500 pc and a group associated with L1217 at a distance of $400 \mathrm{pc}$ (Kun 1998). Both L1217 and L1235 are coincident with the G109+11 infrared loop (Kiss et al. 2006). The small, isolated protostellar core L1221 was included in our survey and is also coincident with the interior of the infrared loop, but its assumed distance of 250 pc places it foreground of L1217 and L1235.

The age of the supernova at the center of the Cepheus Flare Shell is 40,000 yr (Grenier et al. 1989), but the bubble that the shell encloses appears to be significantly older, $7 \mathrm{Myr}$, and was probably a preexisting wind-driven cavity created by a star that subsequently exploded as a supernova (Olano et al. 2006, and discussion therein). Ionization pressure from $\mathrm{H}$ II regions can enhance gravitational collapse in surrounding material, e.g., the Horsehead Nebula (Ward-Thompson et al. 2006), and trigger star formation in the heads of pillar-like filaments, e.g., M16's "Pillars of Creation" (Hester et al. 1996). We conjecture that this mode of star formation may have been responsible for some of the WTTS currently situated inside the Cepheus Void. The Cepheus Flare Shell is an expanding H I ring (Olano et al. 2006), however, and shows negligible $\mathrm{H} \alpha$ emission when compared with the Ceph OB2 H II region (see Figure 19). The lack of $\mathrm{H} \alpha$ emission means that any hot gas associated with the CFS has had sufficient time to cool or escape. 


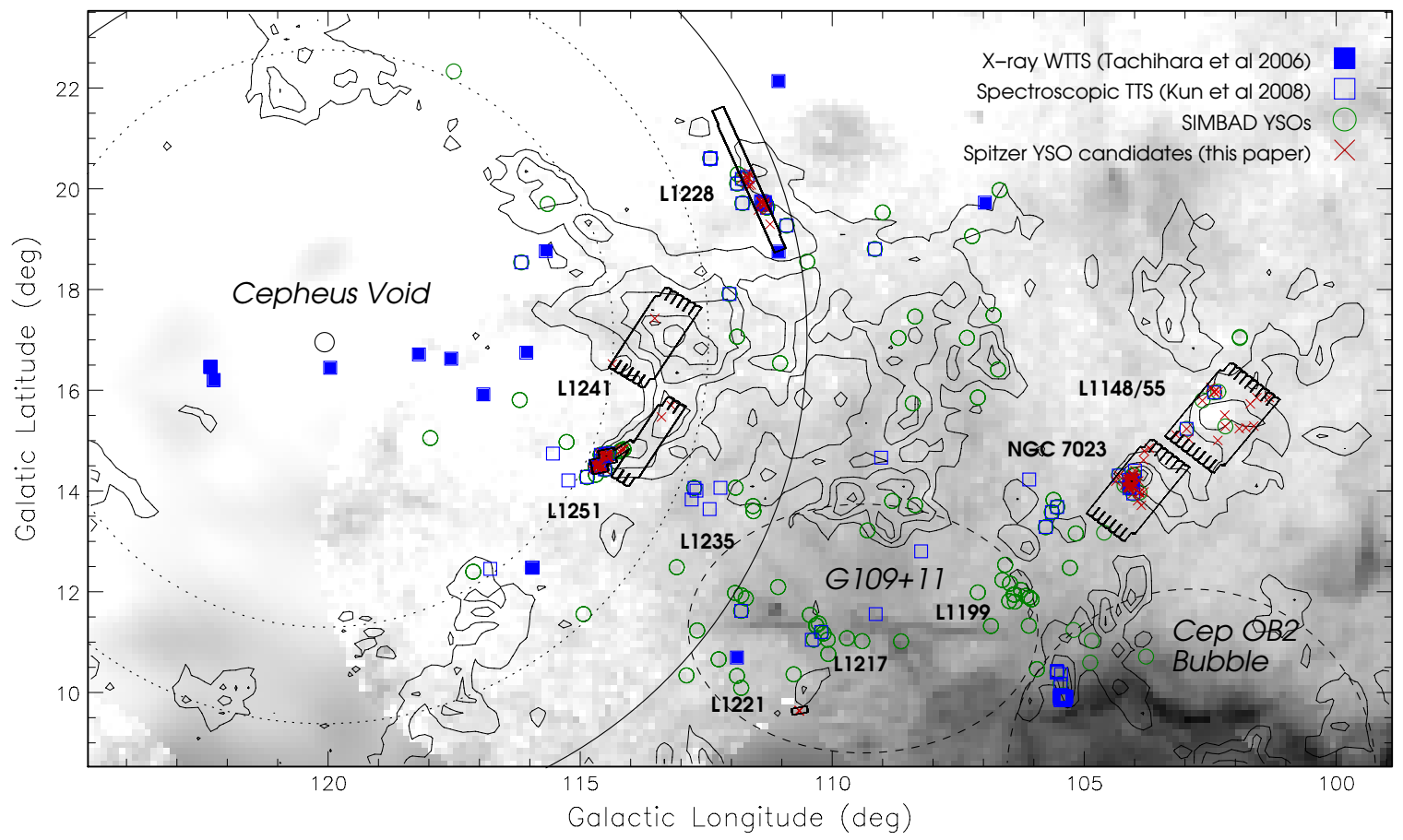

Figure 19. Distribution of YSOs and YSO candidates toward the Cepheus Flare. A key to the different YSO symbol is shown top right. The areas mapped with Spitzer at $24 \mu \mathrm{m}$ are shown by black footprints. The labels are the names of selected Lynds dark nebulae (Lynds 1962). The gray scale shows log scaled H $\alpha$ emission (Finkbeiner 2003). The contours show integrated CO emission at 5, 10, 15, 20, and $25 \mathrm{~K} \mathrm{~km} \mathrm{~s}^{-1}$ (Dame et al. 2001). The solid black line shows the present extent of the Cepheus Flare Shell that surrounds the Cepheus Void (Olano et al. 2006). The two dotted circles show the estimated extent of the Shell 2 Myr and 4 Myr ago. The dashed black lines denote the G109+11 and Cepheus OB2 Bubble.

(A color version of this figure is available in the online journal.)

Table 17

Star-formation Statistics for the Cepheus Flare

\begin{tabular}{lccccccccc}
\hline \hline Boundary & $N_{\text {YSO }}$ & $\begin{array}{c}\Omega^{\mathrm{a}} \\
\left(\mathrm{deg}^{2}\right)\end{array}$ & $\begin{array}{c}A^{\mathrm{b}} \\
\left(\mathrm{pc}^{2}\right)\end{array}$ & $\begin{array}{c}N_{\mathrm{YSO} / \Omega} \\
\left(\mathrm{deg}^{-2}\right)\end{array}$ & $\begin{array}{c}N_{\mathrm{YSO}} / A \\
\left(\mathrm{pc}^{-2}\right)\end{array}$ & $\begin{array}{c}\mathrm{SFR}^{\mathrm{c}} \\
\left(M_{\odot} \mathrm{Myr}^{-1}\right)\end{array}$ & $\begin{array}{c}\mathrm{SFR} / A \\
\left(M_{\odot} \mathrm{Myr}^{-1} \mathrm{pc}^{-2}\right)\end{array}$ & $\begin{array}{c}\mathrm{M}_{\text {cloud }} \\
\left(M_{\odot}\right)\end{array}$ & $\begin{array}{c}\mathrm{M}_{\text {cloud }} / A \\
\left(M_{\odot} \mathrm{pc}^{-2}\right)\end{array}$ \\
\hline$A_{V}<1$ & 19 & 6.2 & 170 & 3.1 & 0.11 & $\ldots$ & $\ldots$ & $\ldots$ & $\ldots$ \\
$A_{V}>1$ & 114 & 2.1 & 59 & 53 & 1.9 & 29 & $\ldots .49$ & $\ldots$ \\
$A_{V}>5$ & 92 & 0.40 & 11 & 230 & 8.4 & 58 & 2.1 & $\ldots$ \\
\hline
\end{tabular}

Notes.

${ }^{a}$ The solid angle enclosed by the contour.

${ }^{\mathrm{b}}$ The cross-sectional area assuming an average distance of $300 \mathrm{pc}\left(1 \mathrm{sqr} \mathrm{deg}=27.4 \mathrm{pc}^{2}\right)$.

${ }^{c}$ The star-formation rate (SFR) calculated assuming a star-formation time scale of $2 \mathrm{Myr}$ and an average stellar mass of $0.5 M_{\odot}$.

Following the Olano et al. (2006) analysis of the dynamics of the CFS, we estimate that its radius was $30 \mathrm{pc}$ at $4 \mathrm{Myr}$ ago and $40 \mathrm{pc} 2 \mathrm{Myr}$ ago. These two radii are shown by the concentric, dotted circles in Figure 19. The current radius of $50 \mathrm{pc}$ is shown by the solid circle. These circles are only sketches of the Shell's extent and ignore differences in the local density of material the Shell was propagating into and the effects of a two-dimensional shape imposed on the projected distribution of a three-dimensional structure. Nevertheless, the isolated Void WTTS are clearly located within the central circle and most of them are within a radius of $4^{\circ}$ (the CFS radius after only $1-$ $2 \mathrm{Myr}$ ). The Void WTTS have ages comparable with the age of the bubble (Tachihara et al. 2005).

The ratio of more evolved YSOs to younger YSOs (Class I+F/Class II+III), i.e., the "class ratio," in protostellar cores $\left(N_{\text {YSO }}<5\right)$ and loose groups $\left(N_{\text {YSO }} \geqslant 5\right)$ is $11 / 9=1.22$ and $28 / 53=0.52$, respectively. The class ratio for the entire region is $35 / 98=0.36$. Taken at face value, this implies that the protostellar cores represent a younger YSO population than the loose groups and that the loose groups have a relatively narrow range of YSO ages. The class ratio for distributed YSOs and for loose YSO groups averaged across the c2d clouds is 0.24 and 0.89 (Evans et al. 2009). The c2d average for distributed YSOs is similar to the class ratio for the entire Cepheus Flare and further supports the idea that relatively distributed star formation is the dominant mode of star formation in this region.

Our SED analysis has shown that the disk population in Cepheus is skewed toward young accreting disks. The age of the disks would be expected if the YSOs in the groups formed simultaneously due to a fairly recent triggering event, such as the passage of a shock wave. The current best estimate for the lifetime of low mass star formation prior to the Class III phase is 2-3 Myr (see Evans et al. 2009, and discussion therein). The four loose YSO groups are in the zone between the current position of the CFS and its estimated position $4 \mathrm{Myr}$ ago. Given that L1251 and L1228 are believed to be on opposite sides of the Cepheus Void (Kun et al. 2008), projection effects could mean 
that L1251 is closer to the Shell wall than it actually appears. Therefore, the general empirical ages of Class II-dominated groups are consistent with their formation at a time when the CFS was coincident with their position.

The Void WTTS (Tachihara et al. 2005) occur at the same Galactic latitude $\left(b \sim 16^{\circ}\right)$ as L1241, but they are distributed toward the projected interior of the Cepheus Void at $115^{\circ}<l<$ $123^{\circ}$. The region on the other side of L1251 and L1228, in the longitude range $107^{\circ}<l<110^{\circ}$, appears in Figure 19 to hold a reservoir of material that has not yet started forming stars in the same manner. L1241 also represents a large mass of material that has not yet started forming stars. It has usually been placed at the same distance as L1251 (see Section 2), but the lack of star formation may indicate that it is actually at a different distance and, like the material in the range $107^{\circ}<l<110^{\circ}$, has yet to encounter the CFS. Alternatively, the $\mathrm{CO}$ emission toward L1241 may be the superposition of two or more low density clouds; Yonekura et al. (1997) list two different components to $V_{\text {LSR }}$ toward L1241.

Simulations of the impact of ionizing radiation on a turbulent ISM show that it can enhance the preexisting density contrasts and the efficiency of star formation (Dale et al. 2007; Gritschneder et al. 2009). This enhancement could explain why we see more efficient star formation in the cores toward the CFS. The surface density of the Gritschneder et al. (2009) simulation at $t=250 \mathrm{kyr}$ (their Figure 1) resembles the distribution of CO seen in the Cepheus Flare (our Figure 19). However, the timescale and physical dimensions of their simulation are shorter and smaller than inferred for the CFS (see above).

Moving farther west, there is a zone clear of $\mathrm{CO}$ emission at $l \sim 106^{\circ}$ and then we reach the locations of L1172+L1174 at $l \sim 104^{\circ}$ and L1148+L1152+L1155 at $l \sim 102^{\circ}$. Even here, farthest from the CFS, it appears that some degree of triggering is at work. The NGC 7023 cluster in L1172 is being shaped by the powerful illumination of HD 200775 and holds the largest concentration of YSOs in the region we have surveyed. We presume that the formation of these YSOs has been triggered by compression of the material around the reflection nebula caused by HD 200775. L1148+L1152+L1155 are comprised of a series of prestellar cores and protostellar cores that contain only a few YSOs each. It would appear that this final region is the most unperturbed, but again there is some evidence of outside influences on the cores (Nutter et al. 2009).

The general scenario for star formation in the Cepheus Flare has the initial conditions of a turbulent ISM where distributed, quiescent, low-efficiency star formation is the norm. Approximately $7 \mathrm{Myr}$ ago, a progenitor high-mass star with a strong circumstellar wind formed at the center of what would become the CFS (Olano et al. 2006). The wind or the radiation from the star compressed the surrounding gas into pillars/filaments in which a series of new YSOs formed. The influence of the progenitor star eventually stripped the natal molecular gas from this generation of YSOs and left them behind as the Cepheus Flare WTTSs (Tachihara et al. 2005). The wind from the progenitor continued building up an expanding shell of material (Olano et al. 2006) until it went supernova approximately $40 \mathrm{kyr}$ years ago (Grenier et al. 1989). A second generation of star formation occurred on surfaces facing the interior of the wind bubble. The passage of the CFS enhanced a core's SFE relative to the original quiescent mode of star formation and produced a series of small YSO groups. The material of the clouds that surrounded the YSO groups was stripped away before the coevolving group, on average, reached the Class III phase.

\section{SUMMARY}

1. We have presented Spitzer IRAC and MIPS observations of the L1148+L1152+L1155, L1172+L1174, L1221, L1228, L1241, and L1247+L1251 dark cloud associations in the Cepheus Flare region $(D \sim 300 \mathrm{pc})$ as part of the SGBS of star formation within $500 \mathrm{pc}$.

2. The SGBS delivery catalog (which is available from the SSC as a legacy data product) contains over 71 thousand sources within the area common to all wavelengths and detected in at least one IRAC band. Of these, 6.5 thousand sources have detections at all four IRAC wavelengths and 889 also have detections at MIPS $24 \mu \mathrm{m}$. Across the entire area, 431 sources were detected at MIPS $70 \mu \mathrm{m}$.

3. Three color-magnitude schemes based on 2MASS+IRAC+ MIPS, 2MASS+MIPS, and IRAC-only photometry were used to reject background galaxies from the catalog. A total of 133 candidate YSOs were identified in this manner. Two-thirds of these were classified by the infrared spectral index as Class II YSOs (generally classical T Tauri YSOs), and one-fifth were classified as Class I YSOs (embedded protostars). Cross-identifications were made for 93 candidate YSOs in the GSC-II and 20 candidate YSOs in the IRAS Faint Source Catalog. The 10 additional previously known YSOs that were not identified by Spitzer were found to be coincident with entries in the catalog.

4. The Cepheus Flare Spitzer luminosity function peaks at $\log \left(L / L_{\odot}\right) \sim-1.5$. Above this value, the luminosity function has a power-law index of 1.6 in agreement with that found for IRAS-only YSO candidates.

5. SED modeling was conducted, following Harvey et al. (2008) and Cieza et al. (2007), to estimate the degree of infrared excess for Class II and Class III YSOs. The majority of the YSOs was found to have accretion-style disks. The values of $A_{\mathrm{V}}$ estimated from the 2MASS $J-K_{s}$ color were overestimated for known variable stars. We suggest that YSO \#83 is a possible transitional disk candidate.

6. A comparison of 2'.5 resolution extinction maps with $160 \mu \mathrm{m}$ emission maps showed 14-18 dense cores (depending on consolidation) split between the six dark cloud associations. Three of these, L1155, L1241, and L1247, are confirmed as starless above the $A_{\mathrm{V}}=5$ contour. It was found that the morphology of the $160 \mu \mathrm{m}$ and higher resolution Spitzer $A_{\mathrm{V}}$ maps agreed particularly well for quiescent prestellar cores, but diverged in less quiescent regions (e.g., the heart of the NGC 7023 reflection nebula).

7. Five YSO associations with peak stellar mass volume densities greater than a theoretical cluster disruption density were found. L1228N, L1228S, L1251B, and L1251A are small groups with 5-15 members each. The larger NGC 7023 cluster contains 32 YSOs and 21 non-YSO members. The star-formation rate for dense cores with and without formal associations of YSOs was found to be $\sim 8 \%$ and $\sim 1 \%$, respectively. No difference in the mass of prestellar and protostellar cores was found.

We are grateful to Maria Kun and her co-authors for a preprint of their chapter, "Star Forming Regions in Cepheus," from the Handbook of Star Forming Regions, Vol 1: The Northern Sky (2008, ed. B. Reipurth, ASP Monograph Series). 
J.M.K. thanks UK STFC for PDRA funding through the Cardiff Astronomy Rolling Grant. Support for this work was provided by NASA through contracts 1330171 and 1365763 (T.L.B.) issued by the Jet Propulsion Laboratory, California Institute of Technology, to the Smithsonian Astronomical Observatory.

This work has made use of data and resources from the Spitzer Space Telescope, the Two Micron All Sky Survey, the Digitized Sky Survey II, the Guide Star Catalog II, the James Clerk Maxwell Telescope, the Canadian Astronomy Data Centre SCUBA Archive, and the SIMBAD database. The Spitzer Space Telescope is operated by the Jet Propulsion Laboratory, California Institute of Technology under a contract with NASA. The Two Micron All Sky Survey is a joint project of the University of Massachusetts and the Infrared Processing and Analysis Center/California Institute of Technology, funded by the National Aeronautics and Space Administration and the National Science Foundation. The Digitized Sky Survey-II is based on photographic data obtained using The UK Schmidt Telescope. The UK Schmidt Telescope was operated by the Royal Observatory Edinburgh, with funding from the UK Science and Engineering Research Council, until 1988 June, and thereafter by the Anglo-Australian Observatory. Original plate material is copyright (c) of the Royal Observatory Edinburgh and the Anglo-Australian Observatory. The plates were processed into the present compressed digital form with their permission. The Digitized Sky Survey was produced at the Space Telescope Science Institute under US Government grant NAG W-2166. The Guide Star Catalog-II is a joint project of the Space Telescope Science Institute and the Osservatorio Astronomico di Torino. Space Telescope Science Institute is operated by the Association of Universities for Research in Astronomy, for the National Aeronautics and Space Administration under contract NAS526555. The participation of the Osservatorio Astronomico di Torino is supported by the Italian Council for Research in Astronomy. Additional support is provided by European Southern Observatory, Space Telescope European Coordinating Facility, the International GEMINI project, and the European Space Agency Astrophysics Division. The James Clerk Maxwell Telescope is operated by The Joint Astronomy Centre on behalf of the Science and Technology Facilities Council of the United Kingdom, the Netherlands Organisation for Scientific Research, and the National Research Council of Canada. SCUBA was built at the Royal Observatory, Edinburgh. The Canadian Astronomy Data Centre is operated by the National Research Council of Canada with the support of the Canadian Space Agency. The SIMBAD database is operated at CDS, Strasbourg, France. Marvelous fellows, all of them.

Facilities: Spitzer, CTIO: 2MASS (), JCMT

\section{APPENDIX}

\section{DISCUSSION OF INDIVIDUAL REGIONS}

The SCUBA Legacy Catalog (Di Francesco et al. 2008) was used to identify the regions of the Cepheus Flare that have been observed with SCUBA. The majority of the cores in the Cepheus Flare have been observed with the SCUBA scan map method at one time or another and with varying levels of signal to noise. The exceptions are L1251W and L1228S, which were not observed, and PV Cep, which has only been observed with the jiggle-map method. The SCUBA Legacy maps have been filtered to remove large-scale angular variations that may or may not be real for any given map (Di Francesco et al. 2008).
The Cepheus Flare scan maps were rereduced by us to compare possible extended structure with the MIPS $160 \mu \mathrm{m}$ emission. The entire set of raw data for the Cepheus Flare scan maps and the jiggle maps for PV Cep and L1172 (which is higher signal to noise than the L1172 scan map) were downloaded from the archive and reduced as described in Section 5.1. A median baseline was used during the reduction of the scan maps. The data have been smoothed to a FWHM resolution of $18^{\prime \prime}$ to improve the image quality.

Figure 20 shows the IRAC $3.6 \mu \mathrm{m}$ images toward 12 Cepheus Flare cores. The IRAC images are shown with a log stretch to emphasize faint nebulosity. The white contours show the SCUBA $850 \mu \mathrm{m}$ data. The contours are at intervals of $2 \sigma(3 \sigma$ for $\mathrm{L} 1228 \mathrm{~N}$ and $10 \sigma$ for PV Cep) and start at $3 \sigma$. The local $1 \sigma$ rms was measured as the point-to-point variation toward offsource regions and was typically $10-30 \mathrm{mJy}$ per $18^{\prime \prime}$ beam. The dashed contours show $A_{\mathrm{V}}$ from Figure 17 at levels of 5, 9, 13, 17, and $21 \mathrm{mag}$. The positions of YSO candidates are shown by star markers and each of them is labeled with their index number. The additional objects of interest are shown by white crosses and are discussed in the following sections. Selected outflows are shown by dashed lines. Table 18 lists the driving sources, Herbig-Haro numbers (where relevant), and references for the outflows shown in Figure 20. The relation of the cores to each other is shown in Figure 1.

\section{A.1. The L1148+L1152+L1155+L1157 Dark Cloud Association}

The $A_{\mathrm{V}}$ and $160 \mu \mathrm{m}$ maps both show a ring- or loop-like structure (see Figures 17 and 18) that includes the dense cores L1148, L1155, L1152, and L1157. This ring is remarkable because three out of these four cores contain relatively isolated Class 0 protostars, suggesting that star formation is still ongoing within this structure. Two bright point sources are visible at $160 \mu \mathrm{m}$, the Class 0 protostar L1157, and the Herbig AeBe star PV Cep.

\section{A.1.1. PV Cep}

PV Cep is a bright Herbig Ae/Be star (Li et al. 1994). Figure 20(a) shows the region around PV Cep. The SCUBA $850 \mu \mathrm{m}$ emission is point-like, but there is a slight vein of low-level emission that points to the south. This could be CO contamination from the outflow in the $850 \mu \mathrm{m}$ waveband (e.g., see Chini et al. 2001). The MIPS $160 \mu \mathrm{m}$ emission is also point-like, but is saturated in the BCD images. The IRAC image shown in Figure 20(a) is a BCD image from the SSC archive and still has some of the artifacts that were removed from other images by the $\mathrm{c} 2 \mathrm{~d} / \mathrm{SGBS}$ processing. An inspection of the SSC images shows that PV Cep is also saturated at all four IRAC bands. Nevertheless, the breadth of additional photometry does allow for a fairly complete reconstruction of its SED as shown in Figure 10. The SED has had to be scaled downward to fit on the same axes as the other SEDs as it is so bright.

The white crosses in Figure 20(a) show the positions of the HH 315 outflow. The closest two HH objects to PV Cep have been connected to show the orientation of the outflow. Lines connecting paired $\mathrm{HH}$ objects on either side of PV Cep do not cross in the same location and were taken by Goodman \& Arce (2004) as evidence that the source was moving to the west. Taken with other evidence, they concluded that PV Cep had an unusually high local motion and has escaped from the NGC 7023 cluster. 
(a) PV Cep

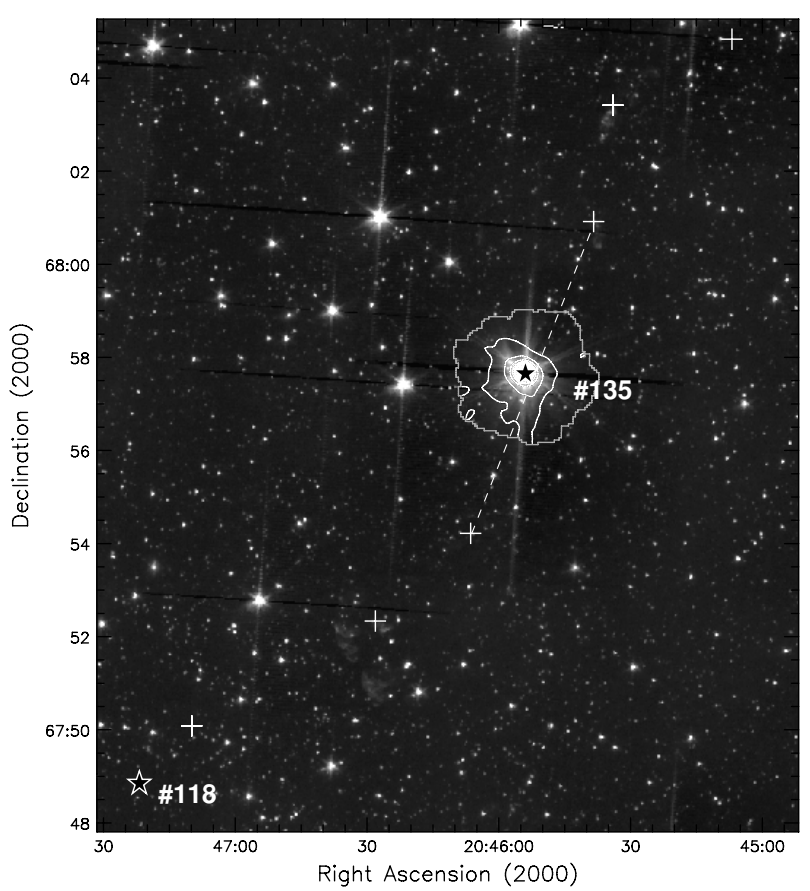

(c) L1152

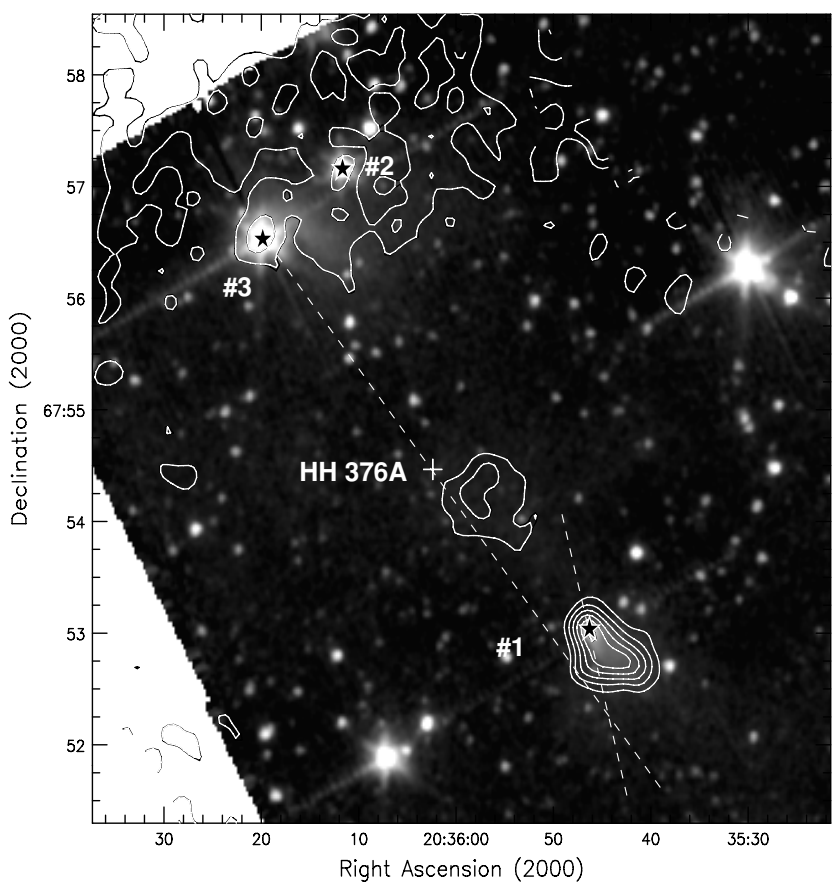

(b) L1148

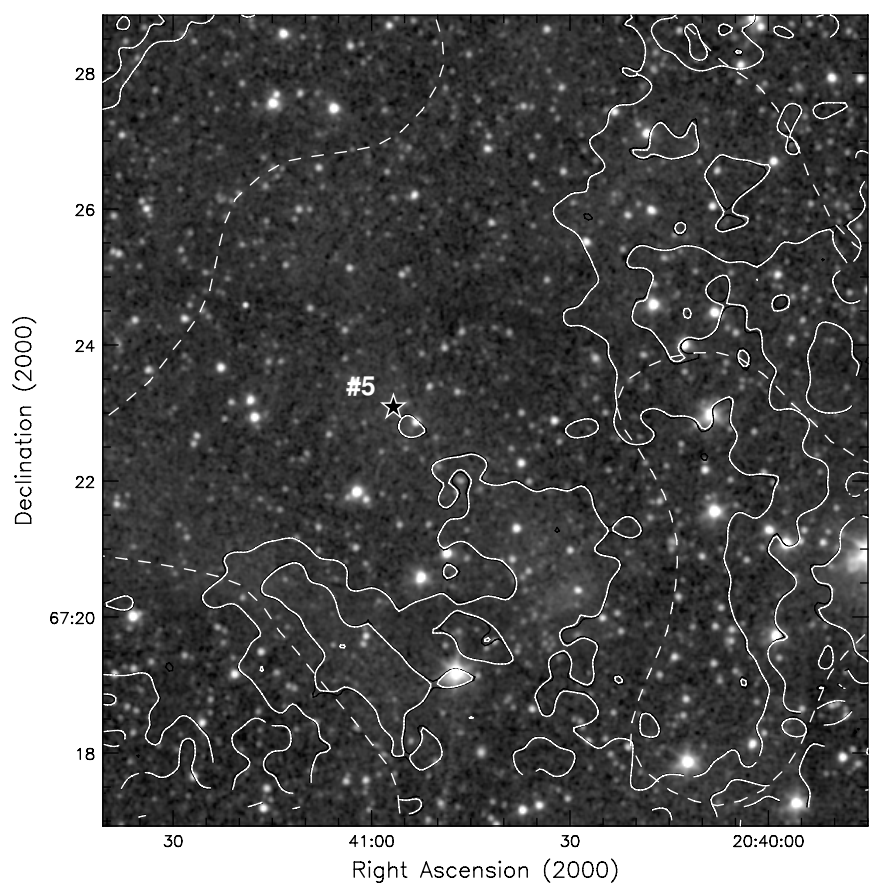

(d) L1155C

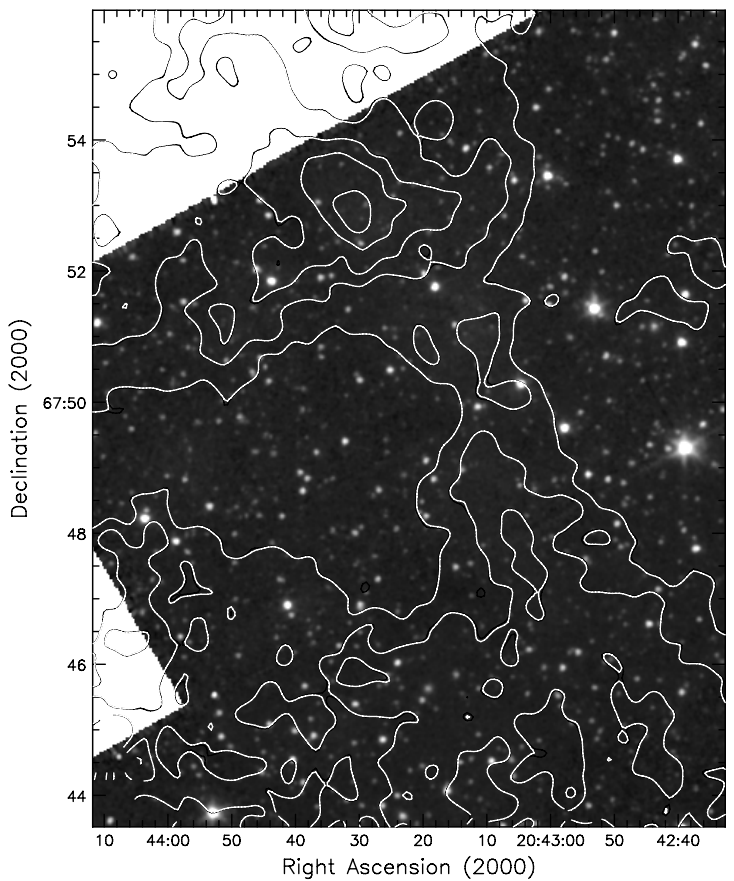

Figure 20. Images of IRAC $3.6 \mu \mathrm{m}$ emission toward the centers of the Cepheus Flare cores. The solid contours show SCUBA $850 \mu \mathrm{m}$ emission in intervals of $2 \sigma$ starting at $3 \sigma$ (intervals of $3 \sigma$ and $10 \sigma$ are used for L1228N and PV Cep, respectively). The edges of the mapped SCUBA areas are shown by the gray contours. The dashed contours show Spitzer $A_{V}$ in intervals of 4 mag starting at 5 mag. The straight dashed lines show the approximate orientation of selected outflows. The positions of YSO candidates are shown by the star markers. These are labeled with the YSO index. The positions of other objects discussed in the main text are shown by the white crosses.

\section{A.1.2. 21148}

Kauffmann et al. (2005) studied L1148 and reported the discovery of a very low luminosity object they called L1148-IRS. To the south of L1148 in Figure 17, the $A_{\mathrm{V}}=5$ contour breaks in two between a northern component containing L1148-IRS and a southern component that runs off the bottom of the high-resolution $A_{\mathrm{V}}$ box. This southern core is L1147.

Figure 20(b) shows the region around L1148 at IRAC $3.6 \mu \mathrm{m}$. Care must be taken in interpreting the SCUBA $850 \mu \mathrm{m}$ data as much of the brighter emission is toward the edge of the map and could easily be Fourier artifacts introduced by the SCUBA scan 
(e) L1157

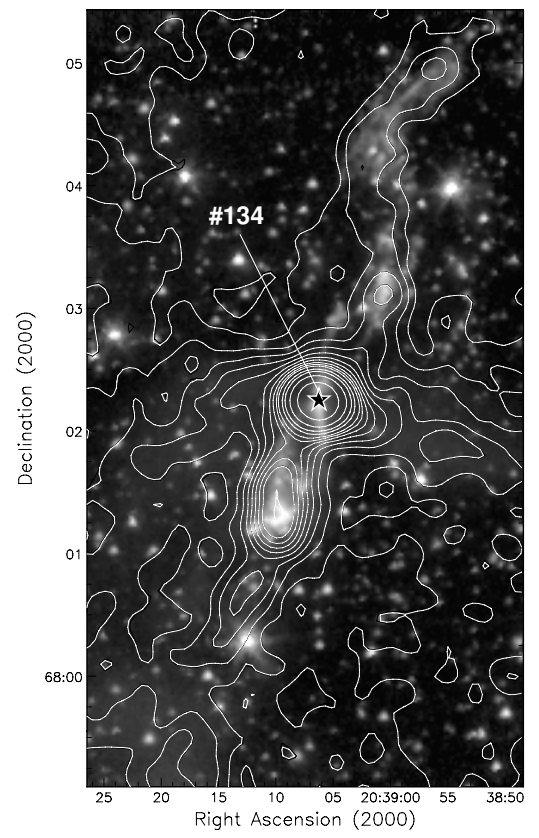

(g) L1174 Enlargement

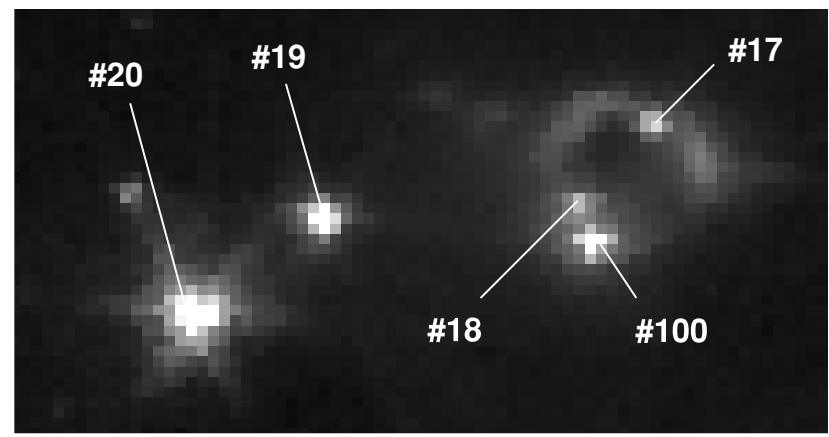

(f) L1174 (NGC 7023)

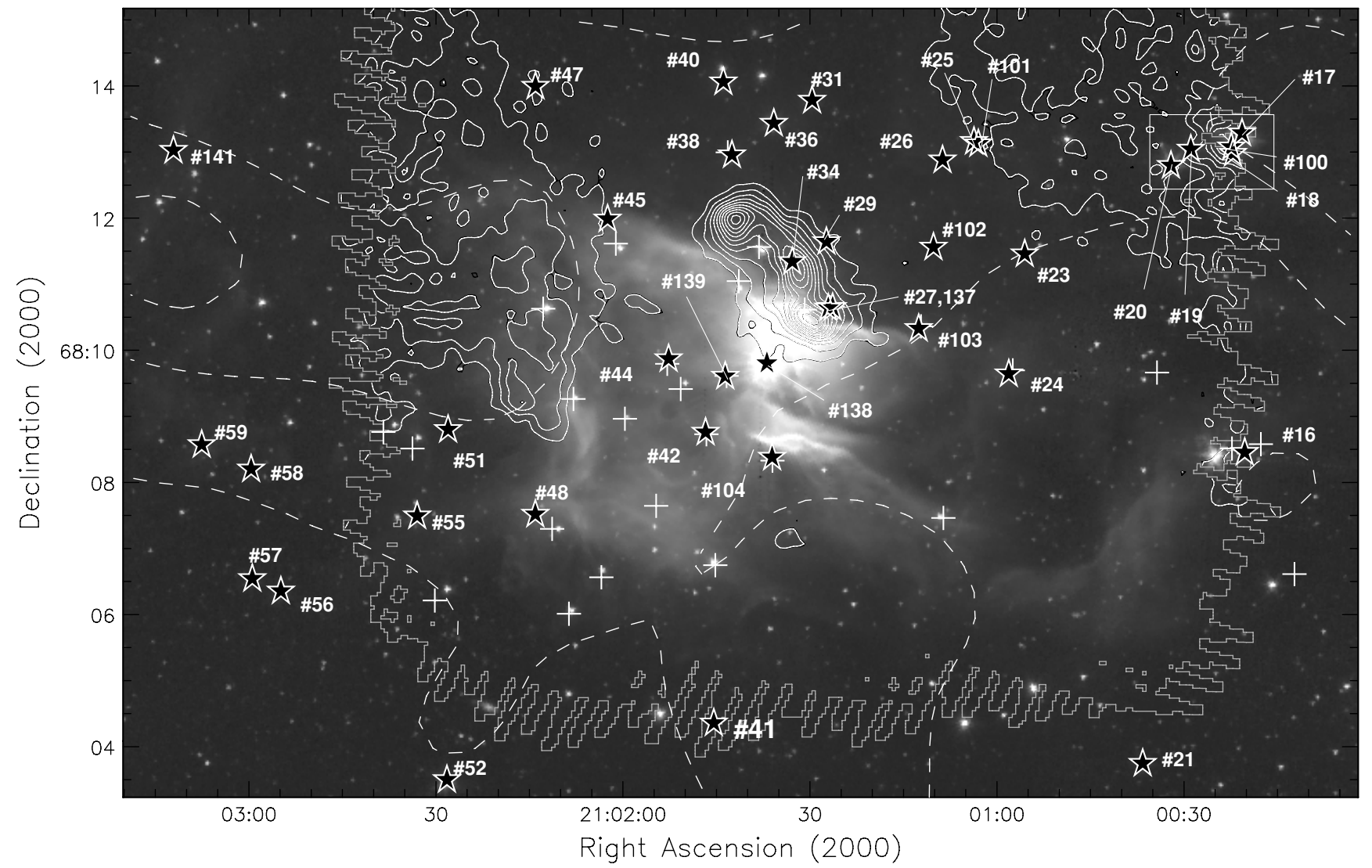

Figure 20. (Continued)

map restoration. A comparison with the MAMBO filaments (Kauffmann et al. 2005, 2008), the $A_{\mathrm{V}}$ contours, the MIPS $160 \mu \mathrm{m}$ emission, and Akari far-IR emission (Nutter et al. 2009), however, shows common features that support the probability that SCUBA is tracing extended structure. Taken together, they appear to show a loop or square of material/emission with
L1148-IRS on the northeastern side. The two MAMBO dust filaments that Kauffmann et al. (2005) observed toward L1148 are also part of this loop. The filament that contains L1148-IRS (YSO \#5 in Figure 20(b)) follows the line of the extinction, but the brighter MAMBO filament is $3^{\prime}$ to the east and is coincident with a $160 \mu \mathrm{m}$ feature. The SCUBA contours also show these 
(h) L1172D

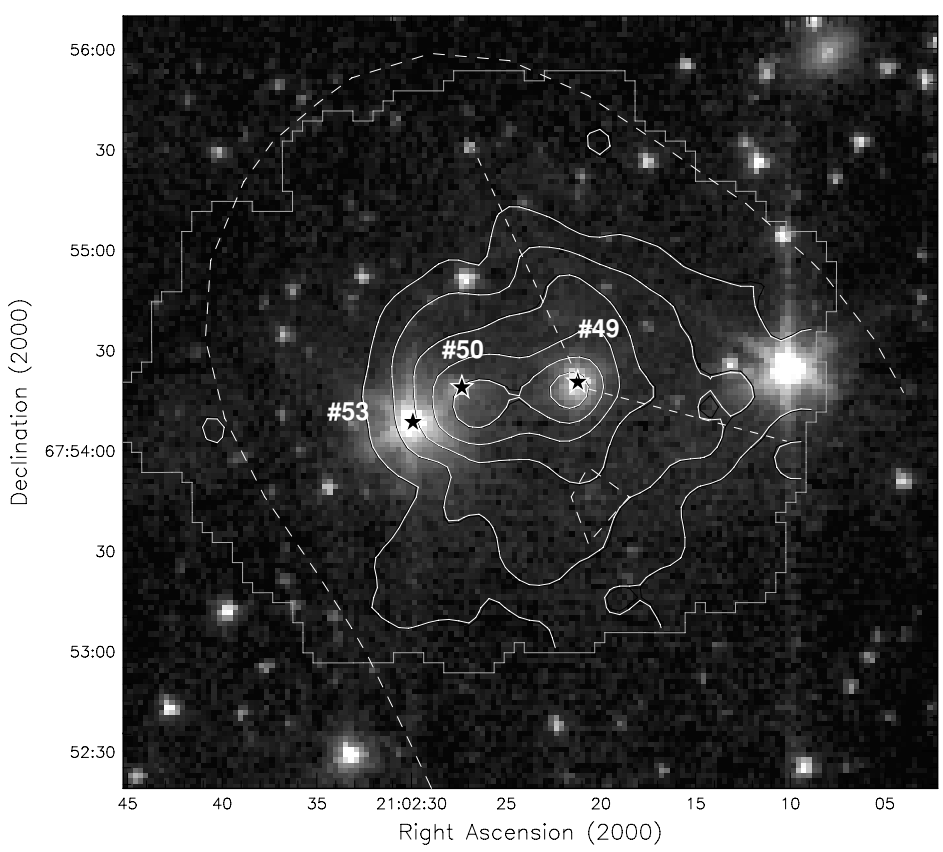

(j) $\mathrm{L} 1228 \mathrm{~N}$

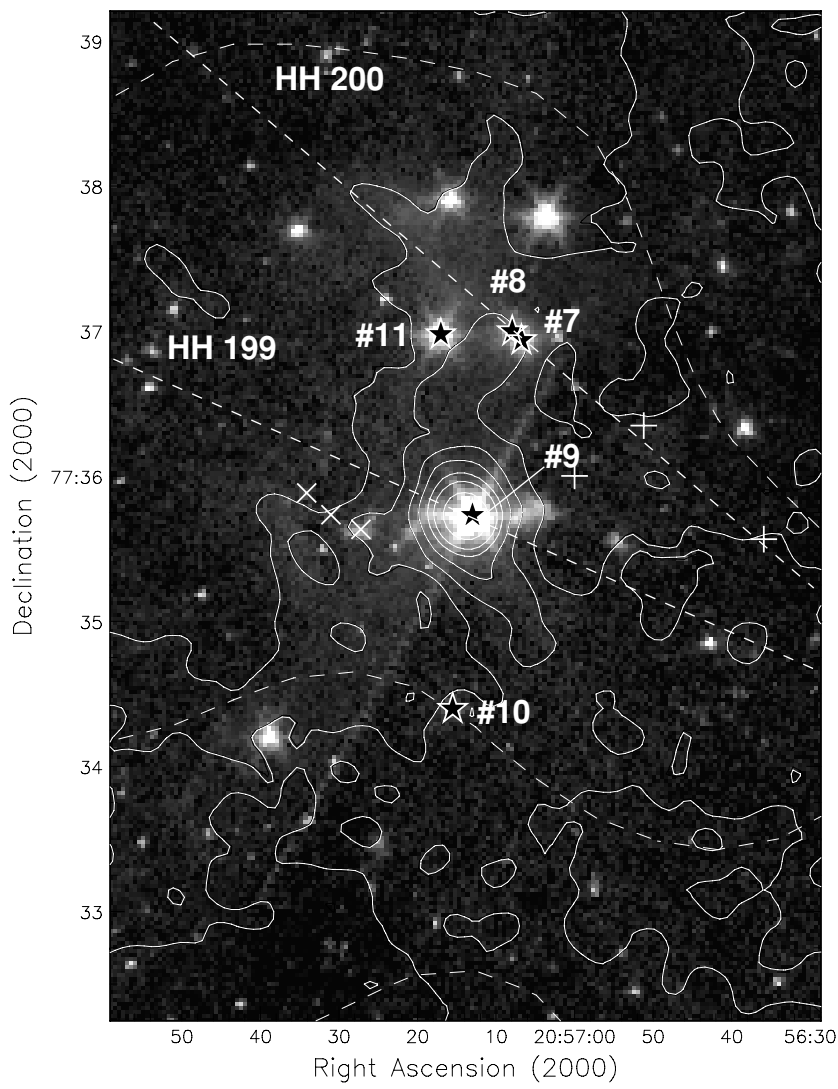

(i) L1221

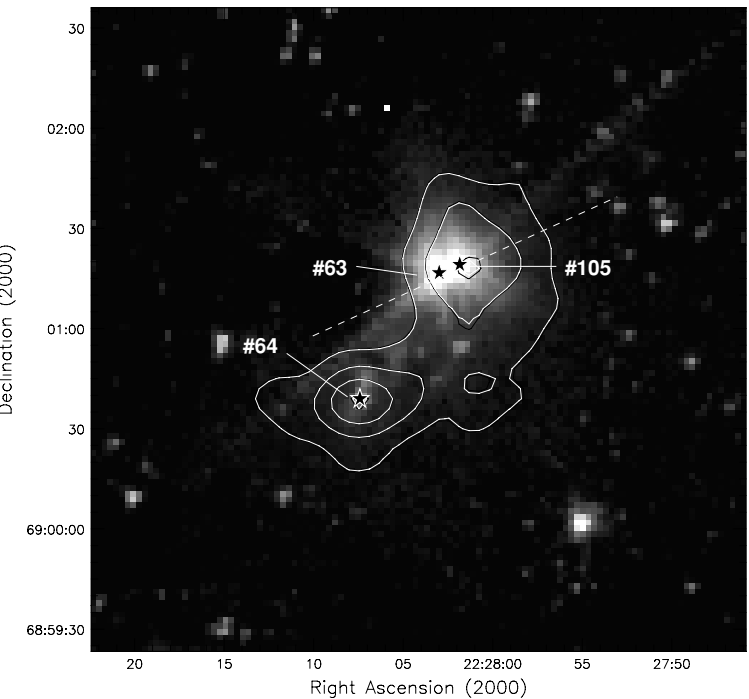

(k) L1228S

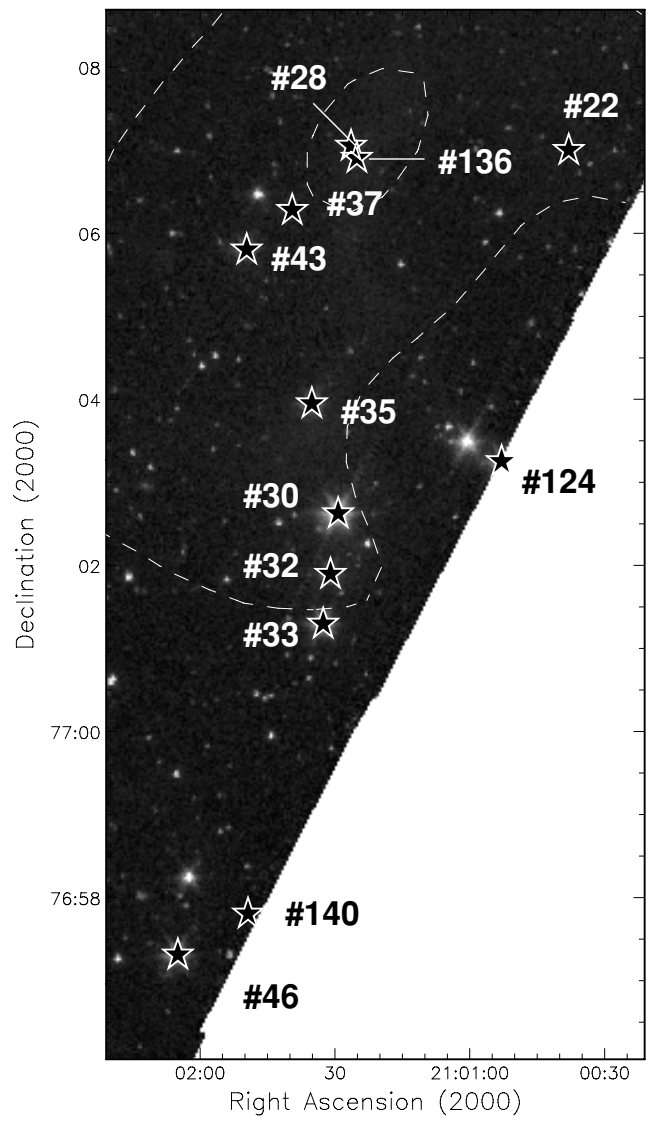

Figure 20. (Continued)

two filaments aligned NE-SW with L1148-IRS at the very top of one of them.

The extinction peaks around L1148-IRS and to the northwest. The $160 \mu \mathrm{m}$ emission also peaks to the northwest, but is relatively weak around L1148-IRS and is stronger toward the south. Nutter et al. (2009) observed the L1148+L1155 filament at 90,140 , and $160 \mu \mathrm{m}$ with the Akari far-infrared satellite. They showed that the extended far-infrared emission toward L1148 and L1155 followed particular edges of regions of high visual extinction and attributed the difference in the two 
(1) L1251A

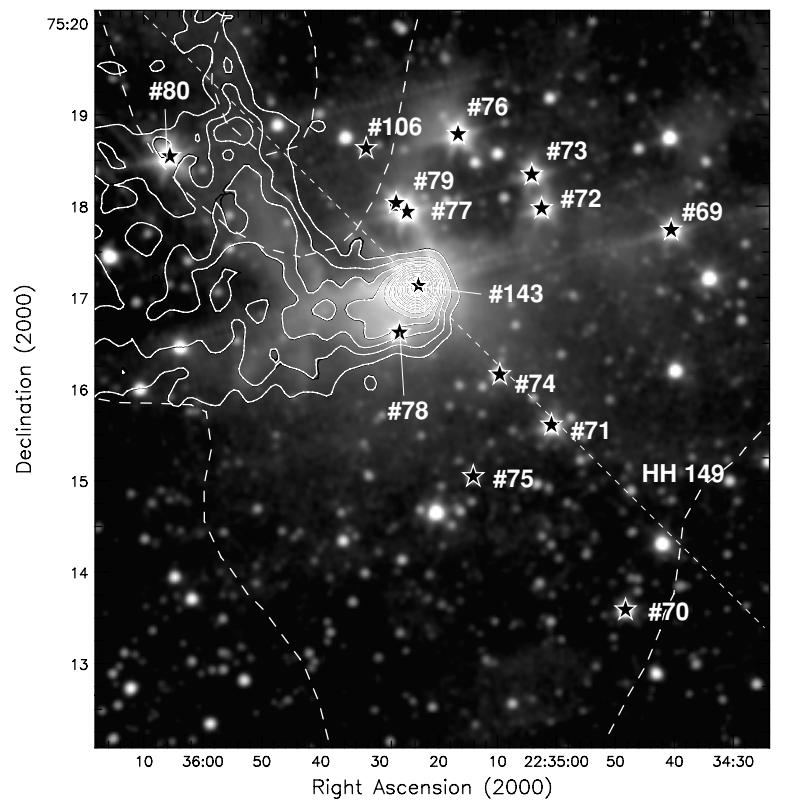

(m) L1251B

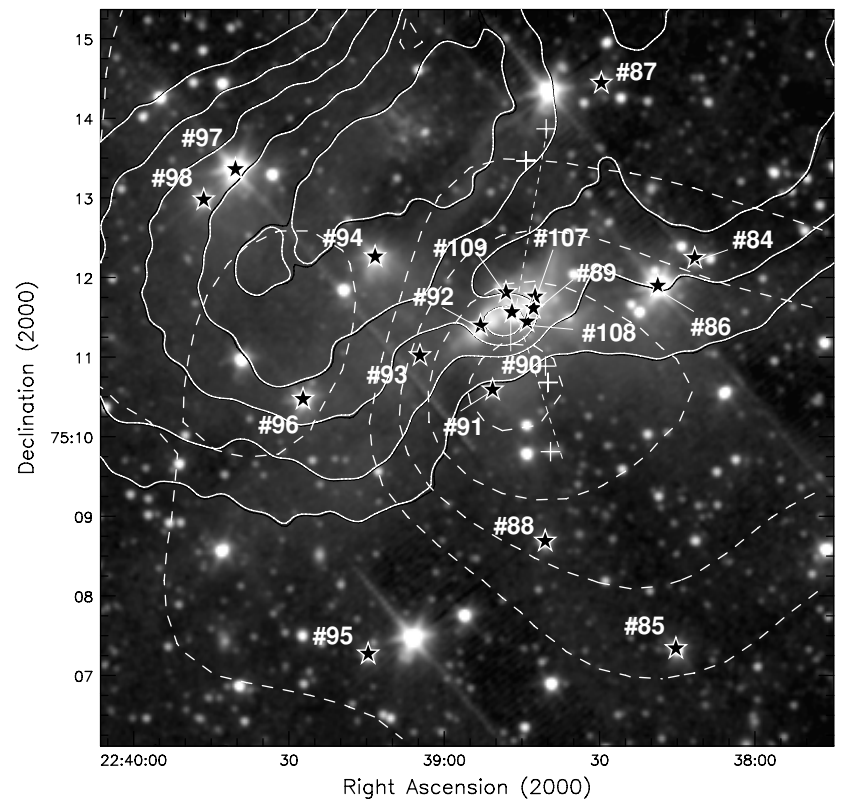

(n) $\mathrm{L} 1251 \mathrm{~W}$

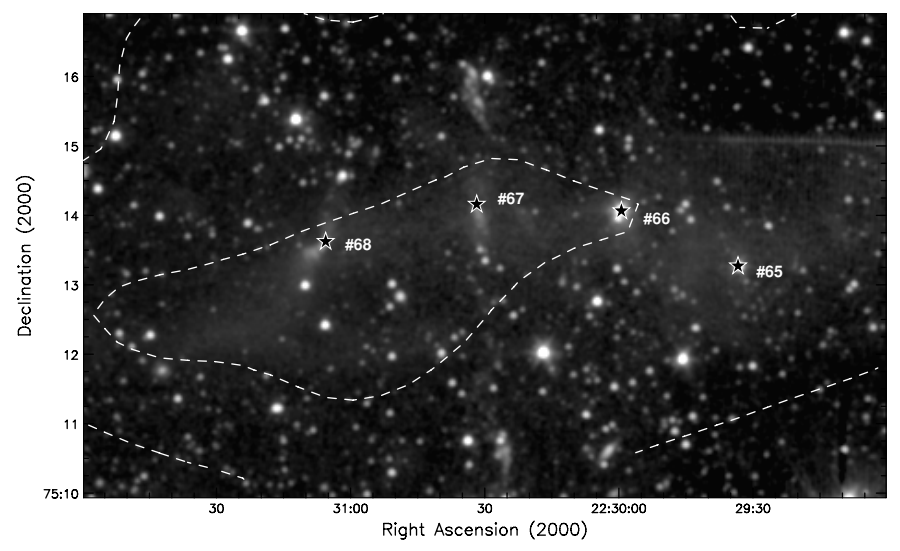

Figure 20. (Continued)

Table 18

Known Outflows in Cepheus

\begin{tabular}{lcccc}
\hline \hline Region & Lit. Name & Assoc. YSO & HH No. & Example References \\
\hline Off-core & PV Cep & 135 & $215,315,415$ & Goodman \& Arce 2004 \\
L1152 & GM 3-12 & 3 & 376 & Reipurth et al. 1997; Movsessian et al. 2004 \\
L1152 & L1152 SMM & 1 & $\ldots$ & Bontemps et al. 1996 \\
L1157 & L1157 IRS & 134 & 375 & Umemoto et al. 1992; Looney et al. 2007 \\
L1172D & L1172 SMM 1 & 49 & $\ldots$ & Myers et al. 1988 \\
L1174 & HD 200775 & 136 & $\ldots$ & Fuente et al. 1998 \\
L1221 & L1221 IRS 1 & 105 & $\ldots$ & Umemoto et al. 1991; Lee \& Ho 2005 \\
L1228N & HH 200 IRS & 7 & 200 & Bally et al. 1995 \\
L1228N & HH 199 1 & 9 & 199 & Bally et al. 1995 \\
L1251B & $\ldots$ & Undetermined & 189 & Eiroa et al. 1994 \\
L1251A & $\ldots$ & 143 & 149 & Sato \& Fukui 1989; Balazs et al. 1992 \\
\hline
\end{tabular}

distributions in L1155 to the effect of an exterior source heating the dust along one side of the core. They also detect the source L1148-IRS strongly at $90 \mu \mathrm{m}$. There is good agreement between the $160 \mu \mathrm{m}$ emission as observed by MIPS and Akari.
A.1.3. $L 1152$

Chapman \& Mundy (2009) observed L1152 with Spitzer as part of their study of the extinction law in four dense cores. They found three YSO candidates in this field-the two Class 
II sources YSO \#2 and \#3 and the Class 0 source YSO \#1. The IRAS sources F20353+6742 and F20358+6746 are associated with YSOs \#1 and \#3. Figure 20(c) shows the region around L1152 at IRAC $3.6 \mu \mathrm{m}$. An alternative reduction of this SCUBA data was originally presented by Young et al. (2006).

The Herbig-Haro $(\mathrm{HH}) 376$ jet cuts through this regionits orientation is shown by the dashed line and the position of HH 376A is indicated (Reipurth et al. 1997). It is immediately noticeable that the HH 376 outflow is aligned tightly with the objects of interest in this field. The northern end of the outflow is coincident with the cometary nebula GM 3-12 (RNO $124=$ YSO \#3); this is a conical nebula with two helical arms (Movsessian et al. 2004). The HH object 376A has a bow shock structure suggesting that it is moving to the S-W away from YSO \#3 (Reipurth et al. 1997; Movsessian et al. 2004). Given the proximity of the isolated group of $850 \mu \mathrm{m}$ contours to $\mathrm{HH}$ $376 \mathrm{~A}$, it is possible that we are seeing the bow-shaped HH376A (Movsessian et al. 2004) plowing into the clump of material that is ahead of it.

YSO \#1 is associated with the peak of the SCUBA emission in the map. It has a $\sim 1^{\prime}$ long bipolar molecular $(\mathrm{CO})$ outflow that is offset from the direction of the HH 376 outflow (Bontemps et al. 1996, shown as the shorter dotted line in Figure 20(c)). The HH 376 outflow could be powered by YSO \#1 (e.g., see Reipurth et al. 1997), but given the offset between it and YSO \#1, this would appear unlikely. The projected line of the $\mathrm{HH}$ outflow, however, is close enough to the dense material seen at $850 \mu \mathrm{m}$ that the outflow could possibly have influenced the evolution of YSO \#1 or even triggered its formation. There is a region of $3.6 \mu \mathrm{m}$ nebulosity that stretches away from YSO \#1 parallel to the direction of the HH 376 outflow. YSO \#1 does show faint nebulosity in JHK, which is clipped to the NE (Heyer et al. 1990; Connelley et al. 2007). Chapman \& Mundy (2009) identify the nebulosity as an outflow that is visible from 2MASS $K_{s}$ to IRAC $8.0 \mu \mathrm{m}$ that is altering the dust grain properties within surrounding material. This nebulosity is coincident with the $850 \mu \mathrm{m}$ contours.

Clark (1991) suggested that YSO \#1 is a very young candidate protostar. The spectral index and $T_{\text {bol }}$ estimates of the classification of YSO \#3 and \#1 agree that the former is more evolved than the latter. YSO \#1 has a value of $T_{\text {bol }}=33 \mathrm{~K}$, suggesting that it is a Class 0 source. It also has $L_{\text {submm }} / L_{\text {bol }}=5.0 \%$, which is above the canonical value dividing Class 0 and Class I sources. YSO \#3 has neither a continuum detection at millimeter wavelengths (e.g., a disk; Terebey et al. 1993) nor a maser detection (Furuya et al. 2003).

\section{A.1.4. $L 1155$}

The $160 \mu \mathrm{m}$ emission in L1155 is fragmented within the $A_{\mathrm{V}}=1$ contour. There is a clear east-west break in the $160 \mu \mathrm{m}$ emission between the smaller eastern core and the western core. The larger western core has two peaks that correspond to the position of L1155C (north peak) and L1155H (southern peak) (Kirk et al. 2005). The smaller eastern core has been listed as either L1155E or L1158 (Kun et al. 2008). The L1155D core is the faint peak of emission slightly to the SE of L1158 (Kirk et al. 2005).

There is general agreement between the distribution of $160 \mu \mathrm{m}$ and $850 \mu \mathrm{m}$ emission toward L1155, as seen with other prestellar cores (Kirk et al. 2007), although the SCUBA emission does peak slightly farther to the north. The strengths of the $\mathrm{L} 1155 \mathrm{C}$ and $\mathrm{L} 1155 \mathrm{H} 160 \mu \mathrm{m}$ peaks are approximately equal. Both the $160 \mu \mathrm{m}$ and $850 \mu \mathrm{m}$ emission run along the side of the low-resolution Dobashi et al. (2005) $A_{\mathrm{V}}$ map. Nutter et al. (2009) used a comparison of Akari, SCUBA, and ISO data to show that there was a monotonic spatial shift of the emission peak with a wavelength that was caused by an edge-to-center negative temperature gradient of $2 \mathrm{~K}$ created by the external illumination of L1155 by the nearby A star BD+671263. This star was one of those used by Straižys et al. (1992) to derive its distance estimate to these cores (see Section 2).

\section{A.1.5. $L 1157$}

The L1157-MM embedded protostar is usually given the same name as the dark cloud. It was not observed by our campaign, but was observed with Spitzer by Looney et al. (2007), who detected a flattened pseudo-disk in absorption at $8 \mu \mathrm{m}$ that matched a similar structure seen in $\mathrm{N}_{2} \mathrm{H}^{+}$and $\mathrm{DCO}^{+}$. The orientation of the disk was perpendicular to the orientation of the YSO's outflow. It was added to our catalog as YSO \#134. It has a bolometric temperature of $37 \mathrm{~K}$, an $L_{\text {submm }} / L_{\text {bol }}$ ratio of $2.8 \%$, and is listed as a Class 0 source in the literature (Kun et al. 2008).

Figure 20(e) shows the region around L1157 at $3.6 \mu \mathrm{m}$ and SCUBA $850 \mu \mathrm{m}$. Both tracers show the same basic pattern, a cross-like structure formed by the N-S outflow and EW nebulosity. The outflow from L1157 has been extensively studied and is considered the "prototype for chemically active outflows" (Kun et al. 2008). Chini et al. (2001) compared $850 \mu \mathrm{m}$ emission with the CO (1-0) emission toward L1157. They saw a similar alignment of the outflow to the $850 \mu \mathrm{m}$ emission and concluded that it must contain significant $\mathrm{CO}$ line contamination.

The $160 \mu \mathrm{m}$ emission across L1157 is dominated by a bright point source centered upon the YSO. Fainter extended emission appears to the SE in the approximate position of the contours in the bottom left corner of Figure 20. There is no such emission to the north of the YSO seen at either $160 \mu \mathrm{m}$ or $850 \mu \mathrm{m}$ and the YSO sits on the northern edge of the core as mapped by the Dobashi $A_{\mathrm{V}}$ extinction. This suggests that the northern portion of the outflow is emerging into a lower density environment than the southern outflow.

\section{A.2. The L1172+L1174 (NGC 7023) Dark Cloud Association}

The L1172+L1174 (NGC 7023) complex is a clustered region of star formation. The extinction map in Figure 17 shows the same T-shaped cloud as Figure 3 with regions of high extinction toward the extremities of the $\mathrm{T}$ and a void near the center. The NGC 7023 reflection nebula, otherwise known as the Iris Nebula, sits at the center of the $\mathrm{T}$ and is powered by the seventh magnitude Herbig AeBe star HD 200775. The $160 \mu$ m emission shows the same basic $\mathrm{T}$ shape, but is dominated by bright emission from the center of the nebula that completely saturates the MIPS detectors. The dark nebula associated with the dense material east and west of NGC 7023 is called L1174. We use the designations L1174A, L1174B, and L1174C to refer to the material to the east, to the west, and in the center of NGC 7023, respectively, based on the decreasing order of peak $A_{\mathrm{V}}$. The dark nebula that forms the T stem is L1172.

Figure 20(f) shows a close-up of the region around the NGC 7023 nebula and cluster. The asymmetric biconical cavity to either side of HD 200775 is clearly visible. The position of HD 200775 is shown by the central source YSO \#138. There is excellent agreement between the lower SCUBA contour and the $A_{\mathrm{V}}$ contours. The SCUBA and extinction contours show the dense material that forms L1174, crossbar of the T, with the reflection nebula immediately to the south. 
The eastern lobe of the nebula appears in bright $3.6 \mu \mathrm{m}$ emission, while only the partial rim on the western lobe appears. The eastern lobe is constrained by a greater level of extinction than the western lobe. The $3.6 \mu \mathrm{m}$ emission traces the southern and western parts of the western lobe, but the northern part is much fainter. This same pattern can be seen in the $160 \mu \mathrm{m}$ map, where the south-west part of the lobe appears in strong emission. Where the broken western lobe encounters the edge of L1174B at $21^{\mathrm{h}} 00^{\mathrm{m}} 20^{\mathrm{s}}+68^{\circ} 13^{\prime} 00^{\prime \prime}$, there is a small group of YSO candidates. This is the NGC 7023 Tight B group as identified in the clustering analysis. An enlargement of these YSOs is shown in the smaller image in Figure 20(g), where they appear to form a small ring-like nebula visible at $3.6 \mu \mathrm{m}$.

L1174C, the central extinction peak just to the north of HD 200775, appears as a short filament with three separate $850 \mu \mathrm{m}$ peaks. The middle peak is coincident with the embedded YSO \#34. It has insufficient data points to calculate $L_{\text {submm }}$, but it has a bolometric temperature of just $14 \mathrm{~K}$, making it a probable Class 0 source. The southern edge of the filament follows the line of a $3.6 \mu \mathrm{m}$ filament. This filament has been mapped with multiple wavelengths, including earlier Spitzer observations, and traces the dissociation front at the end of the dense molecular gas (An \& Sellgren 2003; Werner et al. 2004a). Conditions along the NGC 7023 dissociation front have made it an ideal location to test dust-associated photo-luminescence models (Witt et al. 2006) and emission properties of PAH features (Flagey et al. 2006). Werner et al. (2004a) reported the existence of a small ring of IRAC $4.5 \mu \mathrm{m}$ emission between HD 200775 and the bright ridge just north of it, also seen at $3.3 \mu \mathrm{m}$ by An \& Sellgren (2003). The feature is also present in our maps, but is not highlighted by the stretch we have used to display the images. The top of the bright emission from the nebula forms a sharp dark line that is coincident with a strong linear HI filament (Fuente et al. 1998). The strongest part of the filament is also coincident with the $850 \mu \mathrm{m}$ emission from L1174C.

Figure 20(f) and the extinction map show that the eastern lobe is truncated at $21^{\mathrm{h}} 02^{\mathrm{m}} 10^{\mathrm{s}}$ by the edge of the extinction from L1174A. It is a large block of extinction that runs eastward for about $10^{\prime}$. It is noticeable that star formation is not occurring within this extinction and that all YSO candidate markers are positioned toward its edge or at lower $A_{\mathrm{V}}$.

The molecular gas in NGC 7023 has been extensively studied, but the associated cluster has received relatively little attention (Kun et al. 2008). Sellgren (1983) identified 30 cluster members of various spectral types. We identify nine of Sellgren's cluster members as YSO candidates (these are labeled as such in Table 6). Non-YSO cluster members are shown in Figure 20(f) by white crosses and in Figure 17 by yellow crosses. Sellgren identified seven probable pre-main-sequence stars based on their variability, infrared excess, and hydrogen-line emission. Of those seven, we identify all but the variable stars SX Cep and HZ Cep as YSO candidates.

The majority of the YSO candidates in this region are part of the Tight A Group and the majority of these are situated northward of the two lobes of the nebula in the region coincident with the area of the highest SCUBA emission. The smaller eastern lobe is coincident with several YSOs and stellar cluster members, but the western lobe is noticeably less populated. The majority of the stellar cluster members that do not have YSO candidate cross-identifications are situated to the south and east of HD 200775. The clustering analysis was repeated to include the non-YSO cluster members. The revised $25 M_{\odot} \mathrm{pc}^{-3}$ contour (see Equation (4)) is shown in Figure 17 by the red contour. The inclusion of the non-YSO members extends the Tight A group to cover most of the eastern lobe.

Figure 20(h) shows the L1172D region, which is at the northern tip of the southern extinction maximum in Figure 17. It contains a triplet of YSOs in its densest region. The region was originally mapped with SCUBA by Visser et al. (2002), who labeled the peaks associated with YSO \#49 and \#50 as L1172-SMM1 and L1172-SMM2. They believed L1172-SMM2 to be starless, but the high sensitivity of our IRAC observations has revealed the presence of a faint YSO at that location. The third submillimeter source, L1172-SMM3, is $3^{\prime}$ farther to the south and is confirmed starless. Both SMM1 and SMM2 have very low values of $T_{\mathrm{bol}}, 42 \mathrm{~K}$ and $24 \mathrm{~K}$, respectively, indicating that they are Class 0 sources. SMM1 has a broad $\mathrm{CO}$ outflow that was detected by Myers et al. (1988) and its $L_{\text {submm }} / L_{\text {bol }}$ ratio is greater than $0.5 \%$.

The Class II YSO \#120 (not shown) is coincident with the listed position of L1171 and a small clump of $A_{\mathrm{V}}=1$ extinction.

\section{A.3. $L 1221$}

The protostellar core L1221 lies toward the southern edge of the G109+11 infrared loop (the top of which is shown in Figure 1), closer to the Galactic plane than the other regions in our sample. Figure 20(i) shows two SCUBA sources toward L1221. The northern submillimeter source contains a pair of YSO candidates. Young et al. (2009) first published the Spitzer data for L1221 and detected three infrared sources embedded within the SCUBA emission, which they labeled IRS 1, 2, and 3 . These correspond to the YSO candidates we index as \#63, $\# 105$, and \#64, respectively. YSOs \#63 and \#64 have values of $T_{\text {bol }}$ of $66 \mathrm{~K}$ and $21 \mathrm{~K}$ and $L_{\text {submm }} / L_{\text {bol }}$ ratios of $4 \%$ and $9 \%$, respectively. Based on these $T_{\mathrm{bol}}$ values, YSO \#64 is a definite Class 0 source while YSO \#63 is a Class I source. The approximate orientation of a broad molecular outflow (Lee \& Ho 2005) that has been detected toward L1221 IRS 1 is shown by a dashed line in Figure 20(i). The properties of the SCUBA envelopes around IRS 1 and 3 are very similar and the relative position of the infrared sources to the envelopes are virtually identical, but the protostars themselves are not as similar (Young et al. 2009).

\section{A.4. The L1228 Dark Cloud Association}

The extinction map toward L1228 shows two distinct peaks. A northern peak at a declination of $+77^{\circ} 35^{\prime}$ and a lower $A_{\mathrm{V}}$ southern peak at $+77^{\circ} 08^{\prime}$. We call these two cores L1228N and L1228S, respectively, although we note that L1228N has also been called L1228A in the literature (Kun et al. 2008). L1228N and L1228S each contain small groups of YSO candidates with sufficient stellar densities to be picked up by the clustering analysis. The SIMBAD database shows 10 YSOs in the entire L1228 region, of which four are just outside of the region mapped with Spitzer (all scattered to the west). L1228N has received more attention than $\mathrm{L} 1228 \mathrm{~S}$ and is the site of several Herbig-Haro outflows.

\section{A.4.1. $L 1228 N$}

The extinction map toward L1228N shows a core that is elongated approximately E-W, while the distribution of YSO candidates is approximately N-S. The $160 \mu \mathrm{m}$ map shows a similar morphology, but is dominated by a central bright point source. The source is coincident with YSO \#9 and the position of IRAS 20582+7724. Figure 20(j) shows IRAC and SCUBA 
emission toward the $\mathrm{L} 1228 \mathrm{~N}$ region. It also shows two families of $\mathrm{HH}$ objects as white crosses and the approximate orientation of the HH 199 outflow which emanates from YSO \#9 and the HH 200 outflow which emanates from YSO \#7 (Bally et al. 1995). Additionally, YSO \#9 has an east-west molecular outflow (Arce \& Sargent 2006), which appears as faint nebulosity in the IRAC image. An enhanced image generated from the same IRAC data was shown by Velusamy et al. (2008) as part of their demonstration of the application of the HiRes image deconvolution technique to Spitzer data.

Chapman \& Mundy (2009) examined the mid-infrared extinction in L1228N and concluded that the outflows were altering the extinction law by destroying larger dust grains within the outflow cavities. The four VLA sources were detected in this region at $3.6 \mathrm{~cm}$ by Reipurth et al. (2004). VLA 1 and VLA 4 match the positions of YSO candidates \#9 and \#7 from our survey, but no YSO matches are found for VLA 2 and VLA 3, though they may have IRAC $3.6 \mu \mathrm{m}$ detections. The positions of VLA 2 and VLA 3 are labeled in Figure 20(j).

The SCUBA emission toward L1228 is dominated by a strong point source coincident with the position of YSO \#9 and a similar point source seen at $160 \mu \mathrm{m}$. Surrounding this is low-level emission that is elongated in an N-S direction, perpendicular to the direction of the HH 199 outflow. The eastwest $3.6 \mu \mathrm{m}$ nebulosity surrounding YSO \#9 is coincident with a $2.2 \mu \mathrm{m}$ jet (Bally et al. 1995). The same emission was seen in the $K$ band by Hodapp (1994), who classified the sources surrounding it as a small cluster-albeit at the limits of their definition of a cluster. YSO \#9 has a broad SED, meaning that it is spectrally classified as a flat spectrum source rather than a borderline 0/I source, as would expected by its bolometric temperature (79 K). YSO \#7 is not detected by SCUBA, but has a bolometric temperature $(54 \mathrm{~K})$ below the Class 0 cutoff.

\section{A.4.2. $L 1228 S$}

Figure 20(k) shows L1228S. There are no SCUBA data for this core. The Spitzer observations of L1228S were first presented by Padgett et al. (2004), who showed a cluster of nine sources with Class II or III SEDs. L1228S is situated at the farthest southern extent of the Dobashi $A_{\mathrm{V}}=1$ contour. As noted previously, the L1228S extinction appears to be separated from the $160 \mu \mathrm{m}$ peak. The proximity of L1228S to the edge of the cloud and the proximity of L1228 to the Cepheus Flare Shell leads to the possibility that the cloud around these YSOs is being removed by the passage of the Shell, leaving the YSOs free of their natal cloud (Kun et al. 2008).

\section{A.5. $L 1241$}

L1241 is a relatively massive starless core between the active regions of L1251 and L1228. L1241 is not shown in Figure 20 as it has not been observed with SCUBA and is not associated with any sources in our YSO catalog. It is associated with a large mass of $\mathrm{CO}$ on the west side of the Cepheus Flare (see Figure 1 and Table 1), but it appears to show little evidence for current star formation. Only a single YSO candidate, a Class III YSO at $21^{\mathrm{h}} 56^{\mathrm{m}} 13^{\mathrm{s}} .3+76^{\circ} 58^{\prime} 14^{\prime \prime}$. 2 , was found toward this core, but it is well away from the $A_{\mathrm{V}}$ peak, and this core should still be considered starless. No YSO candidates were found in the SIMBAD database. The nearest SIMBAD YSO is $2^{\circ}$ away ([K98c EM* 61]) and the nearest Tachihara et al. (2005) X-rayselected T Tauri is $3^{\circ}$ away. Why such a large mass of gas is not undergoing star formation is not immediately obvious as the regions L1251 and L1228 seem to be undergoing star formation triggered by the passage of the Cepheus Flare Shell. If L1241 is not undergoing star formation, then it may be because it has not yet encountered the Cepheus Flare Shell and is at a marginally different distance than originally thought.

$$
\text { A.6. } L 1247+L 1251
$$

The map of $A_{\mathrm{V}}$ toward the L1247+L1251 dark cloud association shows a chain of cores proceeding east-west with the peak $A_{\mathrm{V}}$ decreasing towards the east. The cores L1251A, B, and W all contain YSO candidates (see Figure 20(1)-(n)), but L1247 appears starless (not shown). The YSO candidates in this region form three distinct groups: the L1251A and L1251B groups and a small quartet in L1251W. We follow Lee et al. (2006) in naming these cores A and B after the Sato \& Fukui (1989) outflow sources, and we adopt the name $\mathrm{L} 1251 \mathrm{~W}$ to refer to the elongated western core.

The L1247 core has no associated YSOs and is confirmed starless within the sensitivity of our survey. MIPS $160 \mu \mathrm{m}$ was not taken for the $\mathrm{c} 2 \mathrm{~d}$ cores region, so there is no $160 \mu \mathrm{m}$ data available for L1251. Data were taken for L1247; however, it shows excellent correlation between the $160 \mu \mathrm{m}$ emission and the visual extinction. The $160 \mu \mathrm{m}$ emission and the extinction map peak in the same position and show a narrow extension/ filament to the north of the peak.

In L1247+L1251, a total of 39 YSO candidates were identified from the Spitzer photometry. Of these, 16 are not in the SIMBAD database. The majority of these, however, are from the L1251A core and will be discussed in the paper by J.-E. Lee et al. (2009, in preparation). There is a one-to-one correspondence between the YSO candidates \#66, 67, and 68 in L1251A and the ammonia cores T3, T2, and T1 (Tóth \& Walmsley 1996). The outflow HH 189 (Eiroa et al. 1994) is shown by the white crosses and the dashed line in Figure 20(m). It emanates from the tight YSO group associated with the small knot of SCUBA emission, but it is not entirely clear which YSO is driving it. The approximate orientation of the HH 149 is shown by the dashed line in Figure 20(n). The driving source outflow is coincident with YSO \#143 (Sato \& Fukui 1989; Balazs et al. 1992).

The L1251B cluster was studied by Lee et al. (2006) using the same data presented here. They found that a tight cluster of YSO candidates centered around the bright source L1251B IRS 1. See J.-E. Lee et al. (2009, in preparation) for further discussion of this region.

\section{REFERENCES}

Ábrahám, P., Balázs, L. G., \& Kun, M. 2000, A\&A, 354, 645

Alcalá, J. M., et al. 2008, ApJ, 676, 427

Allen, L. E., et al. 2004, ApJS, 154, 363

Allen, L., et al. 2007, in Protostars and Planets V, ed. B. Reipurth, D. Jewitt, \& K. Keil (Tucson, AZ: Univ. of Arizona Press), 361

An, J. H., \& Sellgren, K. 2003, ApJ, 599, 312

André, P., \& Montmerle, T. 1994, ApJ, 420, 837

André, P., \& Saraceno, P. 2005, in ESA Special Publication, Vol. 577, ed. A. Wilson (Noordwijk, Netherlands: ESA), 179

André, P., Ward-Thompson, D., \& Barsony, M. 1993, ApJ, 406, 122

Arce, H. G., \& Sargent, A. I. 2006, ApJ, 646, 1070

Balazs, L. G., Eisloeffel, J., Holl, A., Kelemen, J., \& Kun, M. 1992, A\&A, 255, 281

Bally, J., Devine, D., Fesen, R. A., \& Lane, A. P. 1995, ApJ, 454, 345

Bertout, C., Robichon, N., \& Arenou, F. 1999, A\&A, 352, 574

Bessell, M. S. 1979, PASP, 91, 589

Blair, M., \& Gilmore, G. 1982, PASP, 94, 742

Bohlin, R. C., Savage, B. D., \& Drake, J. F. 1978, ApJ, 224, 132

Bontemps, S., André, P., Terebey, S., \& Cabrit, S. 1996, A\&A, 311, 858

Cartwright, A., \& Whitworth, A. P. 2004, MNRAS, 348, 589

Chapman, N., \& Mundy, L. G. 2009, ApJ, 699, 1866 
Chapman, N. L., et al. 2007, ApJ, 667, 288

Chen, H., Myers, P. C., Ladd, E. F., \& Wood, D. O. S. 1995, ApJ, 445, 377

Chini, R., Ward-Thompson, D., Kirk, J. M., Nielbock, M., Reipurth, B., \& Sievers, A. 2001, A\&A, 369, 155

Christopher, M., Myers, P., Allen, L., Di Francesco, J., \& Megeath, T. 1998, BAAS, 30, 1346

Cieza, L., et al. 2007, ApJ, 667, 308

Clark, F. O. 1991, ApJS, 75, 611

Condon, J. J., Cotton, W. D., Greisen, E. W., Yin, Q. F., Perley, R. A., Taylor G. B., \& Broderick, J. J. 1998, AJ, 115, 1693

Connelley, M. S., Reipurth, B., \& Tokunaga, A. T. 2007, AJ, 133, 1528

Crapsi, A., van Dishoeck, E. F., Hogerheijde, M. R., Pontoppidan, K. M., \& Dullemond, C. P. 2008, A\&A, 486, 245

Currie, T. 2009, AJ, 138, 703

Dale, J. E., Clark, P. C., \& Bonnell, I. A. 2007, MNRAS, 377, 535

Dame, T. M., Hartmann, D., \& Thaddeus, P. 2001, ApJ, 547, 792

Di Francesco, J., Johnstone, D., Kirk, H., MacKenzie, T., \& Ledwosinska, E. 2008, ApJS, 175, 277

Dobashi, K., Uehara, H., Kandori, R., Sakurai, T., Kaiden, M., Umemoto, T., \& Sato, F. 2005, PASJ, 57, 1

Eiroa, C., Torrelles, J. M., Miranda, L. F., Anglada, G., \& Estalella, R. 1994 A\&AS, 108, 73

Emerson, D. T. 1995, in ASP Conf. Ser. 75, Multi-Feed Systems for Radio Telescopes, ed. D. T. Emerson \& J. M. Payne (San Francisco, CA: ASP), 309

Enoch, M. L., Evans, N. J., II, Sargent, A. I., \& Glenn, J. 2009, ApJ, 692, 973

ESA 1997, The Hipparcos and Tycho Catalogues, Tech. Rep. SP-1200, ESA

Evans, N. J., II, et al. 2003, PASP, 115, 965

Evans, N. J., II, et al. 2007, Final Delivery of Data from the c2d Legacy Project: IRAC and MIPS Tech. Rep., SSC/c2d (Pasadena, CA: SSC)

Evans, N. J., II, et al. 2009, ApJS, 181, 321

Fazio, G. G., et al. 2004, ApJS, 154, 10

Finkbeiner, D. P. 2003, ApJS, 146, 407

Flagey, N., Boulanger, F., Verstraete, L., Miville Deschênes, M. A., Noriega Crespo, A., \& Reach, W. T. 2006, A\&A, 453, 969

Fuente, A., Martin-Pintado, J., Rodriguez-Franco, A., \& Moriarty-Schieven, G. D. 1998, A\&A, 339, 575

Furuya, R. S., Kitamura, Y., Wootten, A., Claussen, M. J., \& Kawabe, R. 2003, ApJS, 144, 71

Gladwin, P. P., Kitsionas, S., Boffin, H. M. J., \& Whitworth, A. P. 1999, MNRAS, 302,305

Goodman, A. A., \& Arce, H. G. 2004, ApJ, 608, 831

Gould, B. 1879, Uranometria Argentina, Resultados del Observatorio Nacional Argentino en Cordoba, Vol. 1 (Buenos Aires: P.E. Coni)

Greene, T. P., Wilking, B. A., André, P., Young, E. T., \& Lada, C. J. 1994, ApJ, 434,614

Grenier, I. A., Lebrun, F., Arnaud, M., Dame, T. M., \& Thaddeus, P. 1989, ApJ, 347,231

Gritschneder, M., Naab, T., Walch, S., Burkert, A., \& Heitsch, F. 2009, ApJ, 694, L26

Gutermuth, R. A., Megeath, S. T., Pipher, J. L., Williams, J. P., Allen, L. E., Myers, P. C., \& Raines, S. N. 2005, ApJ, 632, 397

Gutermuth, R. A., et al. 2008, ApJ, 674, 336

Gyul'Budagyan, A. L., \& Magakyan, T. Y. 1977, Astronomicheskij Tsirkulyar, 953,1

Hartmann, L., Megeath, S. T., Allen, L., Luhman, K., Calvet, N., D’ Alessio, P., Franco-Hernandez, R., \& Fazio, G. 2005, ApJ, 629, 881

Harvey, P., Merín, B., Huard, T. L., Rebull, L. M., Chapman, N., Evans, N. J., II, \& Myers, P. C. 2007, ApJ, 663, 1149

Harvey, P. M., et al. 2006, ApJ, 644, 307

Harvey, P. M., et al. 2008, ApJ, 680, 495

Hauschildt, P. H., Allard, F., Ferguson, J., Baron, E., \& Alexander, D. R. 1999, ApJ, 525, 871

Herschel, J. 1847, Results of Astronomical Observations Made at the Cape of Good Hope (London: Smith, Elder, and Co.)

Hester, J. J., et al. 1996, AJ, 111, 2349

Heyer, M. H., Ladd, E. F., Myers, P. C., \& Campbell, B. 1990, AJ, 99, 1585

Hillenbrand, L. A., et al. 2008, ApJ, 677, 630

Hodapp, K.-W. 1994, ApJS, 94, 615

Holland, W. S., et al. 1999, MNRAS, 303, 659

Huard, T., et al. 2009, First Delivery of Data from the Spitzer Gould Belt Legacy Project: IRAC and MIPS Tech. Rep., SSC/SGBS (Pasadena, CA: SSC)

Hubble, E. 1934, ApJ, 79, 8

Jenness, T., \& Lightfoot, J. 1998, SURF-SCUBA User Reduction Facility User's in Manual, Starlink User Note 216.4

Jørgensen, J., Johnstone, D., Kirk, H., Myers, P., Allen, L., \& Shirley, Y. 2008, ApJ, 683, 822
Jørgensen, J. K., et al. 2006, ApJ, 645, 1246

Kauffmann, J., Bertoldi, F., Bourke, T. L., Evans, N. J., II, \& Lee, C. W. 2008, A\&A, 487, 993

Kauffmann, J., Bertoldi, F., \& Evans, N. J., II The C2D Collaboration. 2005, Astron. Nachr., 326, 878

Kenyon, S. J., \& Hartmann, L. 1987, ApJ, 323, 714

Kirk, J. M., Ward-Thompson, D., \& André, P. 2005, MNRAS, 360, 1506

Kirk, J. M., Ward-Thompson, D., \& André, P. 2007, MNRAS, 375, 843

Kiss, C., Moór, A., \& Tóth, L. V. 2004, A\&A, 418, 131

Kiss, Z. T., Tóth, L. V., Krause, O., Kun, M., \& Stickel, M. 2006, A\&A, 453, 923

Kreysa, E., et al. 1999, Infrared Phys. Technol., 40, 191

Kun, M. 1998, ApJS, 115, 59

Kun, M., Kiss, Z. T., \& Balog, Z. 2008, in Handbook of Star Forming Regions, Vol 1: The Northern Sky, ed. B. Reipurth (San Francisco, CA: ASP), 136

Kun, M., \& Prusti, T. 1993, A\&A, 272, 235

Lada, C. J., \& Lada, E. A. 2003, ARA\&A, 41, 57

Lasker, B. M., et al. 2008, AJ, 136, 735

Lee, C.-F., \& Ho, P. T. P. 2005, ApJ, 632, 964

Lee, J.-E., Di Francesco, J., Bourke, T. L., Evans, N. J., II, \& Wu, J. 2007, ApJ, 671,1748

Lee, J.-E., et al. 2006, ApJ, 648, 491

Lemke, D., et al. 1996, A\&A, 315, L64

Li, W., Evans, N. J., II, Harvey, P. M., \& Colome, C. 1994, ApJ, 433, 199

Lonsdale, C. J., et al. 2003, PASP, 115, 897

Looney, L. W., Tobin, J. J., \& Kwon, W. 2007, ApJ, 670, L131

Luhman, K. L., Stauffer, J. R., Muench, A. A., Rieke, G. H., Lada, E. A. Bouvier, J., \& Lada, C. J. 2003, ApJ, 593, 1093

Lynds, B. T. 1962, ApJS, 7, 1

Makovoz, D., Roby, T., Khan, I., \& Booth, H. 2006, Proc. SPIE, 6274, 62740C

McLean, B. J., Greene, G. R., Lattanzi, M. G., \& Pirenne, B. 2000, in ASP Conf. Ser. 216, Astronomical Data Analysis Software and Systems IX, ed. N. Manset, C. Veillet, \& D. Crabtree (San Francisco, CA: ASP), 145

Merín, B., et al. 2008, ApJS, 177, 551

Miville-Deschênes, M.-A., \& Lagache, G. 2005, ApJS, 157, 302

Moshir, M., et al. 1990, in IRAS Faint Source Catalogue, version 2.0, 0

Movsessian, T. A., Magakian, T. Y., Boulesteix, J., \& Amram, P. 2004, A\&A, 413, 203

Myers, P. C., Fuller, G. A., Mathieu, R. D., Beichman, C. A., Benson, P. J., Schild, R. E., \& Emerson, J. P. 1987, ApJ, 319, 340

Myers, P. C., Heyer, M., Snell, R. L., \& Goldsmith, P. F. 1988, ApJ, 324, 907

Myers, P. C., \& Ladd, E. F. 1993, ApJ, 413, L47

Neugebauer, G., et al. 1984, ApJ, 278, L1

Neuhaeuser, R., Sterzik, M. F., Schmitt, J. H. M. M., Wichmann, R., \& Krautter, J. 1995, A\&A, 295, L5

Nutter, D. J., Stamatellos, D., \& Ward-Thompson, D. 2009, MNRAS, 396, 1851

Obayashi, A., Kun, M., Sato, F., Yonekura, Y., \& Fukui, Y. 1998, AJ, 115, 274 Ogura, K., \& Sato, F. 1990, PASJ, 42, 583

Olano, C. A., Meschin, P. I., \& Niemela, V. S. 2006, MNRAS, 369, 867

Oliveira, I., et al. 2009, ApJ, 691, 672

Padgett, D. L., et al. 2004, ApJS, 154, 433

Patel, N. A., Goldsmith, P. F., Heyer, M. H., Snell, R. L., \& Pratap, P. 1998, ApJ, 507, 241

Porras, A., et al. 2007, ApJ, 656, 493

Reach, W. T., et al. 2005, PASP, 117, 978

Rebull, L. M., et al. 2007, ApJS, 171, 447

Reid, I. N., et al. 1991, PASP, 103, 661

Reipurth, B., Bally, J., \& Devine, D. 1997, AJ, 114, 2708

Reipurth, B., Rodríguez, L. F., Anglada, G., \& Bally, J. 2004, AJ, 127, 1736

Rieke, G. H., et al. 2004, ApJS, 154, 25

Robitaille, T. P., Whitney, B. A., Indebetouw, R., Wood, K., \& Denzmore, P. 2006, ApJS, 167, 256

Rodríguez, L. F., \& Reipurth, B. 1996, RevMexAA, 32, 27

Rodríguez, L. F., \& Reipurth, B. 1998, RevMexAA, 34, 13

Rosvick, J. M., \& Davidge, T. J. 1995, PASP, 107, 49

Sato, F., \& Fukui, Y. 1989, ApJ, 343, 773

Sato, F., Mizuno, A., Nagahama, T., Onishi, T., Yonekura, Y., \& Fukui, Y. 1994, ApJ, 435, 279

Schechter, P. L., Mateo, M., \& Saha, A. 1993, PASP, 105, 1342

Sellgren, K. 1983, AJ, 88, 985

Shevchenko, V. S., Ibragimov, M. A., \& Yakubov, S. D. 1989, SvA, 33, 487

Sicilia-Aguilar, A., et al. 2006, ApJ, 638, 897

Simon, T. 2006, AJ, 131, 501

Skrutskie, M. F., et al. 2006, AJ, 131, 1163

Spitzer, L. J. 1958, ApJ, 127, 17 
Straižys, V., Černis, K., Kazlauskas, A., \& Meištas, E. 1992, Balt. Astron., 1, 149

Tachihara, K., Neuhäuser, R., Kun, M., \& Fukui, Y. 2005, A\&A, 437, 919

Terebey, S., Chandler, C. J., \& André, P. 1993, ApJ, 414, 759

Tóth, L. V., Horváth, A., Jr, \& Haikala, L. K. 1995, Ap\&SS, 233, 175

Tóth, L. V., \& Walmsley, C. M. 1996, A\&A, 311, 981

Umemoto, T., Hirano, N., Kameya, O., Fukui, Y., Kuno, N., \& Takakubo, K. 1991, ApJ, 377, 510

Umemoto, T., Iwata, T., Fukui, Y., Mikami, H., Yamamoto, S., Kameya, O., \& Hirano, N. 1992, ApJ, 392, L83

van den Ancker, M. E., The, P. S., Tjin A Djie, H. R. E., Catala, C., de Winter, D., Blondel, P. F. C., \& Waters, L. B. F. M. 1997, A\&A, 324, L33

Velusamy, T., Marsh, K. A., Beichman, C. A., Backus, C. R., \& Thompson, T. J. 2008, AJ, 136, 197

Visser, A. E., Richer, J. S., \& Chandler, C. J. 2002, AJ, 124, 2756

Wainscoat, R. J., Cohen, M., Volk, K., Walker, H. J., \& Schwartz, D. E. 1992, ApJS, 83, 111

Ward-Thompson, D., Motte, F., \& André, P. 1999, MNRAS, 305, 143
Ward-Thompson, D., Nutter, D., Bontemps, S., Whitworth, A., \& Attwood, R 2006, MNRAS, 369, 1201

Ward-Thompson, D., et al. 2007, PASP, 119, 855

Weingartner, J. C., \& Draine, B. T. 2001, ApJ, 548, 296

Werner, M. W., Uchida, K. I., Sellgren, K., Marengo, M., Gordon, K. D., Morris, P. W., Houck, J. R., \& Stansberry, J. A. 2004a, ApJS, 154, 309

Werner, M. W., et al. 2004b, ApJS, 154, 1

Whitcomb, S. E., Gatley, I., Hildebrand, R. H., Keene, J., Sellgren, K., \& Werner, M. W. 1981, ApJ, 246, 416

Wilking, B. A., Lada, C. J., \& Young, E. T. 1989, ApJ, 340, 823

Witt, A. N., Gordon, K. D., Vijh, U. P., Sell, P. H., Smith, T. L., \& Xie, R.-H. 2006, ApJ, 636, 303

Yonekura, Y., Dobashi, K., Mizuno, A., Ogawa, H., \& Fukui, Y. 1997, ApJS, 110,21

Young, C. H., Bourke, T. L., Young, K. E., Evans, N. J., II, Jørgensen, J. K., Shirley, Y. L., van Dishoeck, E. F., \& Hogerheijde, M. 2006, AJ, 132, 1998

Young, C. H., et al. 2009, ApJ, 702, 340 